This manuscript is a preprint which has been submitted for publication. It has not undergone peer review yet. Subsequent versions of this manuscript may have slightly different content. If accepted, the final version of this manuscript will be available via the 'Peer-reviewed Publication DOI' link on the right - hand side of this webpage. Please feel free to contact any of the authors; we welcome feedback. 


\title{
A discontinuous Galerkin method for sequences of earthquakes and aseismic slip on multiple faults using unstructured curvilinear grids
}

\author{
Carsten Uphoff ${ }^{1}$, Dave A. May ${ }^{2}$, Alice-Agnes Gabriel ${ }^{1,2}$ \\ ${ }^{1}$ Department of Earth and Environmental Sciences, Ludwig-Maximilians-University, Munich, Germany \\ ${ }^{2}$ Scripps Institution of Oceanography, UC San Diego, La Jolla, USA
}

Received $\mathrm{xxx}$; in original form $\mathrm{xxx}$

\begin{abstract}
SUMMARY
Physics-based simulations provide a path to overcome the lack of observational data which is hampering a holistic understanding of earthquake faulting and crustal deformation across the vastly varying space-time scales governing the seismic cycle. However, simulations of sequences of earthquakes and aseismic slip (SEAS) including more than one fault, complex geometries, and elastic heterogeneities are challenging. We present a symmetric interior penalty discontinuous Galerkin (SIPG) method to perform SEAS simulations accounting for the complex geometries and heterogeneity of the subsurface. Due to the discontinuous nature of the approximation, the spatial discretisation natively provides a mean to impose boundary and interface conditions associated with geometrically complex domains and embedded faults. The method accommodates two- and three-dimensional domains, is of arbitrary order, handles subelement variations in material properties and supports isoparametric elements, i.e. high-order representations of the exterior and interior boundaries and interfaces including intersecting faults.

We provide an open-source reference implementation, Tandem, that utilises highly efficient kernels for evaluating the SIPG linear and bilinear forms, is inherently parallel and well suited to perform high resolution simulations on large scale distributed memory architectures. Further flexibility and efficiency is provided by optionally defining the displacement evaluation via a discrete Green's function, which is evaluated once in an embarrassingly parallel precomputation step using algorithmically optimal and scalable sparse parallel solvers and preconditioners.

We illustrate the characteristics of the SIPG formulation via an extensive suite of verification problems (analytic, manufactured, and code comparison) for elasto-static and seismic cycle problems. Our verification suite demonstrates that high-order convergence of the discrete solution can be achieved in space and time and highlights the benefits of using a high-order representation of the displacement, material properties, and geometry.

Lastly, we apply Tandem to realistic demonstration models consisting of a 2D SEAS multi-fault scenario on a shallowly dipping normal fault with four curved splay faults, and a 3D multi-fault scenario of elasto-static instantaneous displacement due to the 2019 Ridgecrest, CA, earthquake sequence. We exploit the curvilinear geometry representation in both application examples and elucidate the importance of accurate stress (or displacement gradient) representation on-fault. Our demonstrator models exploit advantages of both the boundary integral and volumetric methods and open new avenues to pursue extreme scale 3D SEAS simulations in the future.
\end{abstract}

Key words: Seismic cycle; Numerical approximations and analysis; Numerical modelling; Transient deformation; Earthquake dynamics; Earthquake interaction, forecasting, and prediction.

\section{INTRODUCTION}

Numerical modelling is an important tool to enhance our understanding of how faults slip, since the spatial and temporal scales involved in (sequences of) earthquakes render the direct observa- tion of earthquake source processes difficult or infeasible (e.g., Lapusta et al. 2019). While historical and geological records have been used to construct catalogs of major earthquakes on known natural fault systems that extend over several thousands of years (Ben-Menahem 1991; Rockwell et al. 2015), the lack of space-time 
complete, quantitative data on fundamental observables of the seismic cycle such as the magnitude and recurrence interval of large earthquakes challenges constructing seismic hazard maps and estimating aftershocks probabilities in the aftermath of large earthquakes (Petersen et al. 2014; Milner et al. 2021). Computational models informed by laboratory experiments and first-order physical principles provide a path to constrain sets of plausible scenarios that extend the knowledge beyond regional statistical laws and enhance our fundamental understanding of earthquake faulting and crustal deformation on different space-time scales.

Numerical simulations of sequences of earthquakes and aseismic slip (termed "SEAS" models) aim to capture the complete seismic cycle in a single self-consistent model (Rice \& Tse 1986; Kato 2002; Lapusta \& Liu 2009; Kaneko et al. 2011; Segall \& Bradley 2012; Barbot et al. 2012; Allison \& Dunham 2018; Abdelmeguid et al. 2019; Mckay et al. 2019; Erickson et al. 2020; Romanet \& Ozawa 2021; Barbot 2021; Jiang et al. 2021). SEAS models consider unified earthquake system dynamics by combining the inter-seismic phase, spontaneous earthquake nucleation as well as co-seismic and post-seismic slip to connect long-term deformation (expressed in fault zone rheology, tectonics, and geometry) and short-term seismicity. In SEAS models, predefined fault surfaces obey a rate- and state-dependent friction law, which welldescribes frictional sliding for many rock types in laboratory experiments (Dieterich 1979; Ruina 1983; Dieterich \& Kilgore 1994). The linear theory of elasticity, ubiquitous in seismology, is used to compute the mechanical response to the displacement discontinuity on-fault and sets shear and normal traction in the rate and state constitutive relation. Often, the required initial and interface conditions governing SEAS models, in particular the loading stresses and constitutive parameters, are not well constrained, necessitating simulations for a wide range of parameters. However, already a single SEAS simulation for one choice of parameters is computationally challenging: Temporal scales vary from milliseconds to years, as a fault may be "locked" for decades, centuries, or millennia, and then rupture within seconds. In space, SEAS models need to resolve hundreds of kilometres of tectonic structures to metrescale resolution on-fault.

Due to the wide variability in time scales, inertia in the elastodynamic relations are often neglected, hence seismic waves are not modelled and transient wave-mediated stress transfer is ignored. A radiation damping term is included in the friction law to mimic the outflow of energy due to seismic waves (Rice 1993). SEAS models that neglect inertia and include radiation damping have been termed quasi-dynamic and can capture the period of stress accumulation and slow movements across faults (e.g., Rice 1993; Segall et al. 2010; Perez-Silva et al. 2021). Quasi-dynamic models quantitatively differ during the co-seismic phase in comparison to fullydynamic models (that include dynamic earthquake rupture) and it is under discussion under which circumstances the qualitative behaviour is affected (Thomas et al. 2014). Models that include inertial effects are typically restricted to idealised scenarios of a single planar fault embedded within a uniform 3D elastic medium (Lapusta \& Liu 2009). Efficient long-term seismicity models considering larger scale fault networks using semi-kinematic approaches have been developed (Tullis et al. 2012; Dieterich et al. 2015) but are approximating or neglecting the effects of inertia effects, slow slip, and inelastic deformation. Switching between fully dynamic and quasi-dynamic approaches is possible but comes at great computational expense (Kaneko et al. 2011; Aagaard et al. 2013).

A variety of numerical discretisation methods have been applied to SEAS type problems, including the boundary integral method (BIM) and boundary element method (BEM) (Barbot 2019; Segall \& Bradley 2012; Bradley 2014; Li \& Liu 2016, 2017; Liu \& Rice 2005; Lapusta et al. 2000; Lapusta \& Liu 2009; Luo et al. 2017), the finite difference method (FDM) (Erickson \& Dunham 2014; Allison \& Dunham 2018; Pranger 2021; Almquist \& Dunham 2021), and the finite element method (FEM) (Aagaard et al. 2013; Kaneko et al. 2011; Kozdon 2019; Luo et al. 2020). In the BEM one only needs to discretise the domain boundaries and the fault (or only the fault for specific geometries such as the halfspace), such that the dimensionality of the problem is effectively reduced by one. However, this method relies on the existence of the analytic fundamental solution (Chen \& Zhou 2010), which is typically only available for homogeneous material parameters and single, planar faults. Therefore, BEM is not applicable for scenarios in general heterogeneous media (e.g., Erickson \& Dunham 2014; Thakur et al. 2020). While heterogeneous material parameters can be easily included in the FDM and FEM, these methods inherently require more degrees of freedom than BEM since they discretise the volume.

In this paper, we evaluate the symmetric interior penalty Galerkin (SIPG) method, which is a discontinuous Galerkin finite element method (DG-FEM) with a particular choice of numerical flux (Arnold et al. 2002), on unstructured curvilinear grids for SEAS models. The discontinuous Galerkin (DG) method for SEAS problems is promising for the following reasons:

- The method is geometrically flexible, meaning there are few restrictions on the complexity and shape of the fault geometry. Such flexibility is realised as the method permits the domain to be decomposed into a set of triangles or quadrilaterals (2D), or a set of tetrahedra or hexahedra (3D), and allows for unstructured meshes to be used. Static spatial adaptivity can be applied to bridge spatial scales (e.g., Mazzieri et al. 2013; Ulrich et al. 2022).

- Values defined on the faces of each cell are double-valued such that the DG function spaces naturally include the displacement discontinuity at the (mesh-aligned) fault. There is no need to split nodes at the fault or to introduce Lagrange multipliers as is the case in the continuous Galerkin FEM (Kaneko et al. 2011; Aagaard et al. 2013). These characteristics of the DG scheme have been successfully exploited in modelling faults in dynamic rupture applications (e.g., Tago et al. 2012; Pelties et al. 2014).

- The polynomial degree of the basis function can be chosen flexibly and the implementation is agnostic with respect to the polynomial degree. Thus, we obtain an arbitrary high-order method on unstructured grids. When high-order convergence is achieved for sufficiently smooth solutions, less degrees of freedom are required in comparison to low-order methods. Establishing whether highorder convergence can be achieved for SEAS problems using SIPG is central to this paper.

- DG methods are provably stable for a wide range of physical processes governed by elliptic, parabolic and hyperbolic partial differential equations (e.g., Hill 1973; Cockburn \& Shu 1989; Grote et al. 2006; Hesthaven \& Warburton 2008; Rivière 2008; Dumbser \& Käser 2006; Reinarz et al. 2020; Duru et al. 2022).

- The physical characteristics of quasi-dynamic SEAS require an incredibly large number of time-steps to be performed (in the order of million time-steps). To enable both high resolution 3D simulations and rapid-to-solution, parallelism must be exploited. The DG discretisation is well suited to complex large scale simulations and scalable up to the largest-available supercomputers (Wilcox et al. 2010; Heinecke et al. 2014; Kronbichler et al. 2017; Uphoff 
et al. 2017; Kronbichler \& Kormann 2019; Schoeder et al. 2019; Arndt et al. 2020; Krenz et al. 2021).

The evaluation of the SIPG method is realised through our open-source reference implementation Tandem, which exploits all of the aforementioned benefits. The novelty and functionality of the computational SEAS framework can be summarized as follows:

(i) Support for 2D and 3D spatial discretisations using unstructured meshes comprised of triangles (2D) and tetrahedra (3D);

(ii) Support for high-order (curvilinear) representation of exterior boundaries and interfaces (faults);

(iii) Sub-element (high-order accurate) representation of material properties and sub-element (high-order accurate) representation of slip, slip-rate in SEAS problems;

(iv) Efficient kernels for the assembly of discontinuous Galerkin operators and residual evaluation;

(v) A fully parallel implementation, including mesh loading, solution stage, output and visualisation;

(vi) Access to state-of-the art solvers, preconditioners and time integrators by using the Portable, Extensible Toolkit for Scientific Computation (PETSc);

(vii) Support to optionally compute the discrete Green's function (i.e., the affine function which maps slip to traction) via efficient scalable (algorithmic and parallel) solvers.

The remainder of this paper is organized as follows. We begin with reviewing the quasi-dynamic SEAS model in Section 2. The SIPG method supporting the slip boundary condition is introduced in Section 3, and analyzed in Section 4. Specific aspects of the DG implementation on curvilinear meshes are presented in Section 5 and coupling to rate-and-state friction is discussed in Section 6. In Section 7 we show how the discrete analogue to a Green's function can be obtained. Results of our numerical experiments are reported in Sections 8 and 9, with the SIPG verification presented in Section 8 and the 2D SEAS splay fault and 3D elasto-static Ridgecrest demonstrators presented in Section 9. We discuss our results in Section 10 and conclude in Section 11.

\section{QUASI-DYNAMIC SEAS MODEL}

We here present the equations of the quasi-dynamic SEAS model. The equations are formulated for $D=2$ and $D=3$, i.e. for two and three space dimensions. We note that summation over lower indices (but not upper indices) appearing twice is implied throughout the paper.

\subsection{Rate and state friction}

In the framework of rate and state friction the effective coefficient of friction $F$ depends on the slip rate vector $\boldsymbol{V}$ and the scalar state variable $\psi$, such that fault strength $\tau_{S}$ is given by

$\tau_{S}=\sigma_{n} F(|\boldsymbol{V}|, \psi)$,

where $\sigma_{n}$ is the normal stress and |.| denotes the Euclidean norm of a vector. The evolution of the state variable is governed by the following ordinary differential equation (ODE):

$\frac{\mathrm{d} \psi}{\mathrm{d} t}=G(|\boldsymbol{V}|, \psi)$.

The slip-rate vector has $D-1$ components, since the slip-rate normal to the fault is defined to be zero (we ignore fault opening). The basis of the slip-rate vector is given by the $D-1$ unit tangents of the fault. By stacking the tangents in the $D \times(D-1)$ matrix $B$ we relate the slip-rate vector to the velocity vector $\boldsymbol{v}$ as $\llbracket v_{i} \rrbracket=$ $B_{i j} V_{j}$. The jump operator $\llbracket$. $\rrbracket$ is defined as $\llbracket v_{i} \rrbracket:=v_{i}^{-}-v_{i}^{+}$and we define that the normal vector $\boldsymbol{n}$ points from the "-" side to the "+" side of a fault discontinuity.

With the conventions adopted, the fault strength is related to shear traction $\tau$ and slip-rate by (Day et al. 2005)

$$
|\boldsymbol{\tau}| \leq \tau_{S}
$$

$\tau_{S} \boldsymbol{V}+\boldsymbol{\tau}|\boldsymbol{V}|=0$.

The first equation limits the traction by the fault strength and the second equation ensures that traction is antiparallel to slip-rate. Moreover, it follows from the second equation that either slip-rate is zero or the magnitude of shear traction is equal to fault strength. That is, if $|\boldsymbol{V}| \neq 0$ then

$-\boldsymbol{\tau}=\sigma_{n} F(|\boldsymbol{V}|, \psi) \frac{\boldsymbol{V}}{|\boldsymbol{V}|}$.

In this paper, we use the regularized version of the rate and state friction law (Rice \& Ben-Zion 1996; Lapusta et al. 2000)

$F(V, \psi)=a \operatorname{arcsinh}\left(\frac{V}{2 V_{0}} \exp \left(\frac{\psi}{a}\right)\right)$

and the Dieterich-Ruina ageing law

$G(V, \psi)=\frac{b V_{0}}{L}\left(\exp \left(\frac{f_{0}-\psi}{b}\right)-\frac{V}{V_{0}}\right)$,

where $a, b, V_{0}, f_{0}, L$ are (possibly space-dependent) constants. We note that the form of the ageing law in Eq. (7) is equivalent to the more familiar form $\mathrm{d} \theta / \mathrm{d} t=1-V \theta / L$ with the substitution $\psi=$ $f_{0}+b \ln \left(V_{0} \theta / L\right)$. However, we prefer (7) which results in the state variable being order one, i.e. $\psi \sim \mathcal{O}(1)$.

\subsection{Quasi-dynamic approximation}

The theory of linear elasticity is used to compute the mechanic response to slip on a fault. Inertia is neglected in the elastodynamic equations in the quasi-dynamic approach such that one needs to solve the elliptic problem

$-\frac{\partial \sigma_{i j}(\boldsymbol{u})}{\partial x_{j}}=f_{i}$,

where $\sigma_{i j}$ is the stress, $\boldsymbol{u}$ the displacement and $f_{i}$ is the bodyforce vector. Given the isotropic stiffness tensor $c_{i j k l}=\lambda \delta_{i j} \delta_{k l}+$ $\mu\left(\delta_{i k} \delta_{j l}+\delta_{i l} \delta_{j k}\right), \lambda$ and $\mu$ are the Lamé parameters and $\delta$ is the Kronecker symbol, the linear stress-strain relation is

$\sigma_{i j}(\boldsymbol{u})=c_{i j k l} \varepsilon_{k l}(\boldsymbol{u})=\frac{1}{2} c_{i j k l}\left(\frac{\partial u_{k}}{\partial x_{l}}+\frac{\partial u_{l}}{\partial x_{k}}\right)$.

Slip is imposed as an interface condition. The slip vector $\boldsymbol{S}$ on the fault is related to the displacement vector $\boldsymbol{u}$ by $\llbracket u_{i} \rrbracket=B_{i j} S_{j}$. Moreover, the free surface is modelled with a Neumann boundary condition and a Dirichlet boundary condition imposes tectonic loading.

Given the displacement $\boldsymbol{u}$ that solves the boundary value problem, one computes the shear traction $\boldsymbol{T}$ in the fault-aligned basis with $T_{i}=T_{i}^{0}+B_{j i} \sigma_{j k}(\boldsymbol{u}) n_{k}$ and the compressive normal stress with $\sigma_{n}=\max \left(0, \sigma_{n}^{0}-n_{i} \sigma_{i j}(\boldsymbol{u}) n_{j}\right)$. Here, the loading stress field is included with $T_{i}^{0}$ and $\sigma_{n}^{0}$. One might consider requiring $\boldsymbol{\tau}=\boldsymbol{T}$, however, the resulting system of equations diverges (Rice 1993). Instead, the radiation damping term $-\eta \boldsymbol{V}$ is added to mimic the outflow of energy due to seismic waves, with $\eta$ being half the 
shear wave impedance. That is, one requires $\boldsymbol{T}=\boldsymbol{\tau}-\eta \boldsymbol{V}$ such that

$-\boldsymbol{T}=\sigma_{n} F(|\boldsymbol{V}|, \psi) \frac{\boldsymbol{V}}{|\boldsymbol{V}|}+\eta \boldsymbol{V}$.

We note that the radiation damping term appears naturally when considering the exact Riemann problem at the fault, which can be used to implement provable stable fault boundary conditions for the fully dynamic equations (Duru \& Dunham 2016; Uphoff 2020).

\subsection{Summary of equations}

We denote the physical domain by $\Omega$ and its boundary by $\partial \Omega$. We assume that $\Omega$ is defined in $\mathbb{R}^{\mathbb{D}}$, where $D=2$ or $D=3$. The boundary of the domain $\partial \Omega$ is partitioned in three pairwise disjoint sets: a Dirichlet boundary $\Gamma^{D}$, Neumann boundary $\Gamma^{N}$, and internal fault boundary $\Gamma^{F}$,

On $\Gamma_{F}$ we solve the following rate and state friction relations

$-T_{i}=\sigma_{n} F(|\boldsymbol{V}|, \psi) \frac{V_{i}}{|\boldsymbol{V}|}+\eta V_{i}$,

$\frac{\mathrm{d} \psi}{\mathrm{d} t}=G(|\boldsymbol{V}|, \psi)$

$\frac{\mathrm{d} S_{i}}{\mathrm{~d} t}=V_{i}, \quad$ where

$T_{i}=T_{i}^{0}+B_{j i} \sigma_{j k}(\boldsymbol{u}) n_{k}$,

$\sigma_{n}=\max \left(0, \sigma_{n}^{0}-n_{i} \sigma_{i j}(\boldsymbol{u}) n_{j}\right)$,

and the displacement is the solution of the boundary value problem

$$
\begin{aligned}
-\frac{\partial \sigma_{i j}(\boldsymbol{u})}{\partial x_{j}} & =f_{i} & & \text { in } \Omega, \\
\sigma_{i j}(\boldsymbol{u}) & =c_{i j k l \varepsilon_{k l}(\boldsymbol{u})} & & \text { in } \Omega, \\
u_{i} & =g_{i}^{D} & & \text { on } \Gamma^{D}, \\
\sigma_{i j}(\boldsymbol{u}) n_{j} & =0 & & \text { on } \Gamma^{N}, \\
\llbracket u_{i} \rrbracket & =g_{i}^{F}=B_{i j} S_{j} & & \text { on } \Gamma^{F} .
\end{aligned}
$$

\section{SYMMETRIC INTERIOR PENALTY GALERKIN}

In this section, we develop a discontinuous Galerkin (DG) method to solve the elasticity sub-problem (12) numerically. Discontinuous Galerkin schemes for elliptic PDEs are typically stated either in the flux formulation or in the primal formulation (Arnold et al. 2002). Central to the flux formulation is the numerical flux which approximates the stress tensor and the displacement on the boundary of an element. The numerical flux is typically single-valued but this is not a necessity. We introduce a double-valued numerical flux that is equivalent to the numerical flux of the symmetric interior penalty Galerkin (SIPG) method, except on the fault $\Gamma^{F}$. We then derive the primal formulation from the flux formulation. The primal formulation defines a variational problem common in finite elements, i.e. it is stated in terms of a bilinear and a linear form, and is useful to assemble the stiffness matrix.

\subsection{Flux formulation}

We note that $c_{i j r s} \frac{\partial u_{r}}{\partial x_{s}}=c_{i j r s} \varepsilon_{k l}(\boldsymbol{u})$ due to the symmetries in the stiffness tensor $\left(c_{i j r s}=c_{i j s r}\right)$ and rewrite the elasticity PDEs as the first-order system

$$
\begin{aligned}
\sigma_{i j}-c_{i j r s} \frac{\partial u_{r}}{\partial x_{s}} & =0, \\
-\frac{\partial \sigma_{i j}}{\partial x_{j}} & =f_{i} .
\end{aligned}
$$

The domain $\Omega$ is approximated using a conforming curvilinear mesh $\mathcal{T}_{h}=\{E\}$. The mesh is defined via mapping $\boldsymbol{X}_{E}: \widehat{E} \rightarrow E$ from the reference element $\widehat{E}$ to the physical element $E$. We require that $\boldsymbol{X}_{E}$ is invertible. The reference element $\widehat{E}$ is given by

$\widehat{E}=\left\{\boldsymbol{\xi}: 0 \leq \boldsymbol{\xi}, \sum_{i=1}^{D} \xi_{i} \leq 1\right\}$,

thus we have a reference triangle for $D=2$ and a reference tetrahedron for $D=3$.

The mapping relates the quantities $(\boldsymbol{\sigma}, \boldsymbol{u})$ on the physical element to the quantities $(\widehat{\boldsymbol{\sigma}}, \widehat{\boldsymbol{u}})$ on the reference element via the following transformation:

$\boldsymbol{\sigma}=\left(\widehat{\boldsymbol{\sigma}}\left(\boldsymbol{\nabla} \boldsymbol{X}_{E}\right)^{-1}\right) \circ \boldsymbol{X}_{E}^{-1}, \quad \boldsymbol{u}=\widehat{\boldsymbol{u}} \circ \boldsymbol{X}_{E}^{-1}$.

We introduce the finite element spaces

$$
\begin{aligned}
& \Sigma_{h}=\left\{\boldsymbol{\sigma} \in\left[L^{2}(\Omega)\right]^{D \times D}: \widehat{\left.\boldsymbol{\sigma}\right|_{E}} \in\left[P_{N}(\widehat{E})\right]^{D \times D} \forall E \in \mathcal{T}_{h}\right\}, \\
& V_{h}=\left\{\boldsymbol{u} \in\left[L^{2}(\Omega)\right]^{D}: \widehat{\left.\boldsymbol{u}\right|_{E}} \in\left[P_{N}(\widehat{E})\right]^{D} \forall E \in \mathcal{T}_{h}\right\},
\end{aligned}
$$

where $P_{N}(\widehat{E})$ is the space of polynomials of degree at most $N$ on $\widehat{E}$. Note that we have the inclusion $\nabla_{h} V_{h} \subset \Sigma_{h}$, where $\nabla_{h}$ takes the gradient element-wise.

We multiply (13) with test functions $\boldsymbol{\tau} \in \Sigma_{h}$ and $\boldsymbol{v} \in V_{h}$, integrate over element $E$, integrate by parts, and introduce numerical fluxes $\widetilde{\sigma}_{i j}$ and $\widetilde{u}_{i}$ :

$$
\begin{array}{r}
\int_{E} \tau_{i j} \sigma_{i j} \mathrm{~d} \boldsymbol{x}+\int_{E} \frac{\partial\left(\tau_{i j} c_{i j r s}\right)}{\partial x_{s}} u_{r} \mathrm{~d} \boldsymbol{x}-\int_{\partial E} \tau_{i j} c_{i j r s} \widetilde{u}_{r} n_{s} \mathrm{~d} s=0 \\
\int_{E} \frac{\partial v_{i}}{\partial x_{j}} \sigma_{i j} \mathrm{~d} \boldsymbol{x}-\int_{\partial E} v_{i} \widetilde{\sigma}_{i j} n_{j} \mathrm{~d} s=\int_{E} v_{i} f_{i} \mathrm{~d} \boldsymbol{x} .
\end{array}
$$

We need a few definitions in order to define the numerical flux. First, let $\Gamma:=\bigcup_{E \in \mathcal{T}_{h}} \partial E$ be the set of facets. The set of interior faces is given by $\Gamma^{i}:=\Gamma \backslash \partial \Omega$ and the set of non-fault interior faces is given by $\Gamma^{0}=\Gamma^{i} \backslash \Gamma^{F}$. Second, we define averages and jumps. Let $E_{1}^{e}$ and $E_{2}^{e}$ be two elements that share an edge $e \in \Gamma^{i}$, that is, $e=E_{1}^{e} \cap E_{2}^{e}$. Then the average and jump of a quantity $v$ along $e$ is given by

$\left\{\{v\}=\frac{1}{2}\left(\left.v\right|_{E_{1}^{e}}+\left.v\right|_{E_{2}^{e}}\right), \quad \llbracket v \rrbracket=\left.v\right|_{E_{1}^{e}}-\left.v\right|_{E_{2}^{e}}\right.$.

When the normal $\boldsymbol{n}^{e}$ is included in the average or jump we define that it is oriented from $E_{1}^{e}$ to $E_{2}^{e}$. For convenience, we also define average and jump for $e \in \partial \Omega$ :

$\left\{\{v\}=\left.v\right|_{E_{1}^{e}}, \quad \llbracket v \rrbracket=\left.v\right|_{E_{1}^{e}}\right.$.

The numerical flux we adopt is equivalent to the usual SIPG numerical flux (Arnold et al. 2002; Rivière 2008), except on the fault. For $e \in \Gamma^{F}$ we define the fluxes according to

$$
\begin{aligned}
\widetilde{\sigma}_{i j} & =\left\{\left\{c_{i j r s} \frac{\partial u_{r}}{\partial x_{s}}\right\}\right\}-\delta_{e}\left(\llbracket u_{i} \rrbracket-g_{i}^{F}\right) n_{j}, \\
\left\{\left\{\widetilde{u}_{i}\right\}\right\} & =\left\{\left\{u_{i}\right\}\right\}, \\
\llbracket \widetilde{u}_{i} \rrbracket & =g_{i}^{F} .
\end{aligned}
$$


Table 1. Summary of the numerical flux. The numerical flux is doublevalued on $\Gamma^{0} \cup \Gamma^{F}$ and single-valued on $\Gamma^{D} \cup \Gamma^{N}$.

\begin{tabular}{cccc}
\hline Interior & $\tilde{\sigma}_{i j}$ & $\left\{\left\{\widetilde{u}_{i}\right\}\right.$ & $\llbracket \widetilde{u}_{i} \rrbracket$ \\
\hline$e \in \Gamma^{0}$ & $\left\{\left\{c_{i j r s} \frac{\partial u_{r}}{\partial x_{s}}\right\}-\delta_{e} \llbracket u_{i} n_{j} \rrbracket\right.$ & $\left\{\left\{u_{i}\right\}\right.$ & 0 \\
$e \in \Gamma^{F}$ & $\left\{\left\{c_{i j r s} \frac{\partial u_{r}}{\partial x_{s}}\right\}-\delta_{e}\left(\llbracket u_{i} \rrbracket-g_{i}^{F}\right) n_{j}\right.$ & $\left\{\left\{u_{i}\right\}\right.$ & $g_{i}^{F}$ \\
\hline Boundary & $\widetilde{\sigma}_{i j}$ & $\widetilde{u}_{i}$ & \\
\hline$e \in \Gamma^{D}$ & $c_{i j r s} \frac{\partial u_{r}}{\partial x_{s}}-\delta_{e}\left(u_{i}-g_{i}^{D}\right) n_{j}$ & $g_{i}^{D}$ & \\
$e \in \Gamma^{N}$ & 0 & $u_{i}$ & \\
\hline
\end{tabular}

By allowing the displacement flux to be double-valued we can prescribe the fault slip $g_{i}^{F}$. Moreover, in the stress flux, we add a penalty term that becomes active when the displacement discontinuity differs from the prescribed slip. The penalty parameter $\delta_{e}$ needs to be large enough to ensure stability. In Section 4 we give a lower bound on $\delta_{e}$. All numerical fluxes used are summarised in Table 1.

\subsection{Primal formulation}

Arnold et al. (2002) explain in detail how to derive the primal formulation from the flux formulation. Inserting our numerical flux and following their procedure (using $\nabla_{h} V_{h} \subset \Sigma_{h}$ ) we obtain

$$
\begin{aligned}
& \sum_{E \in \mathcal{T}_{h}} \int_{E} c_{i j r s} \frac{\partial u_{r}}{\partial x_{s}} \frac{\partial v_{i}}{\partial x_{j}} \mathrm{~d} \boldsymbol{x}+\sum_{e \in \Gamma^{i} \cup \Gamma^{D}} \int_{e} \delta_{e} \llbracket v_{i} \rrbracket \llbracket u_{i} \rrbracket \mathrm{d} s \\
& -\sum_{e \in \Gamma^{i} \cup \Gamma^{D}} \int_{e}\left(\llbracket u_{i} \rrbracket\left\{\left\{c_{i j r s} \frac{\partial v_{r}}{\partial x_{s}}\right\}\right\}+\llbracket v_{i} \rrbracket\left\{\left\{c_{i j r s} \frac{\partial u_{r}}{\partial x_{s}}\right\}\right\}\right) n_{j} \mathrm{~d} s \\
& =\int_{\Omega} f_{i} v_{i} \mathrm{~d} \boldsymbol{x}+\sum_{e \in \Gamma^{D}} \int_{e} \delta_{e} v_{i} g_{i}^{D} \mathrm{~d} s+\sum_{e \in \Gamma^{F}} \int_{e} \delta_{e} \llbracket v_{i} \rrbracket g_{i}^{F} \mathrm{~d} s \\
& -\sum_{e \in \Gamma^{F}} \int_{e} g_{i}^{F}\left\{\left\{c_{i j r s} \frac{\partial v_{r}}{\partial x_{s}} n_{j}\right\}\right\} \mathrm{d} s-\sum_{e \in \Gamma^{D}} \int_{e} g_{i}^{D} c_{i j r s} \frac{\partial v_{r}}{\partial x_{s}} n_{j} \mathrm{~d} s .
\end{aligned}
$$

From (22) we collect all terms which are function of both the test and trial function spaces (LHS of (22)) into a single bilinear form $a(\boldsymbol{u}, \boldsymbol{v})$. The linear form, denoted by $L(\boldsymbol{v})$, collects all terms which are only a function of the test function space (RHS of (22)). The variational problem can be then stated as: Find $\boldsymbol{u}_{h} \in V_{h}$ which satisfies

$a\left(\boldsymbol{u}_{h}, \boldsymbol{v}\right)=L(\boldsymbol{v}), \quad \forall \boldsymbol{v} \in V_{h}$.

Finally, we note that for $\Gamma^{F}=\emptyset$ and $g_{i}^{D}=0$ the bilinear form $a\left(\boldsymbol{u}_{h}, \boldsymbol{v}\right)$ and the linear form $L(\boldsymbol{v})$ is equivalent to the SIPG scheme for elasticity given by Rivière (2008, Chapter 5).

\section{COERCIVITY AND BOUNDEDNESS}

The penalty $\delta_{e}$ needs to be chosen large enough such that the bilinear form $a(\boldsymbol{u}, \boldsymbol{v})$ is coercive, because it follows from coercivity that (23) has a unique solution (Rivière 2008). However, the condition number of the matrix $\boldsymbol{A}$ resulting from (23) increases with the penalty parameter (Antonietti \& Houston 2011). That is, $\delta_{e}$ is ideally set to the lowest value that still ensures coercivity.

Explicit expressions for the penalty parameter are given by Shahbazi (2005) and Epshteyn \& Rivière (2007) for a Poisson model problem. In both papers, numerical experiments demonstrate that the estimate is sharp, i.e. the lowest penalty parameter is close to its theoretical value. The estimates of the penalty parameter rely on the inverse trace inequality (Warburton \& Hesthaven 2003)

$\forall u \in P_{N}(K):\|u\|_{e} \leq \sqrt{\frac{(N+1)(N+D)}{D} \frac{|e|}{|K|}}\|u\|_{K}$,

where $K$ is a planar $D$-simplex, $|K|$ is the volume of $K, e$ is a facet of $K$, and $|e|$ is the length $(D=2)$ or area $(D=3)$ of $e$.

In the following, we find bounds for the penalty parameter for linear elasticity. Similarly to Shahbazi (2005) and Epshteyn \& Rivière (2007) we restrict the analysis to affine mapping functions, because there are some unresolved difficulties for curvilinear coordinates. At the end of this section, we remark on these difficulties.

For the analysis of coercivity, we introduce the semi-norm

$|\boldsymbol{u}|_{c, h}=\left(\sum_{E \in \mathcal{T}_{h}} \int_{E} \frac{\partial u_{i}}{\partial x_{j}} c_{i j r s} \frac{\partial u_{r}}{\partial x_{s}} \mathrm{~d} \boldsymbol{x}\right)^{1 / 2}$

and the energy norm

$\|\boldsymbol{u}\|_{*}=\left(|\boldsymbol{u}|_{c, h}^{2}+\sum_{e \in \Gamma^{i} \cup \Gamma^{D}} \int_{e} \delta_{e} \llbracket u_{i} \rrbracket \llbracket u_{i} \rrbracket \mathrm{d} s\right)^{1 / 2}$.

Moreover, we assume that the stiffness tensor is bounded, i.e. let $\kappa$ be a symmetric $D \times D$ matrix then we have on element $E$

$\forall \boldsymbol{\kappa} \neq 0: 0<c_{0}^{E} \kappa_{i j} \kappa_{i j} \leq \kappa_{i j} c_{i j k l} \kappa_{k l} \leq c_{1}^{E} \kappa_{i j} \kappa_{i j}$.

The constants $c_{0}^{E}$ and $c_{1}^{E}$ are given by

$c_{0}^{E}=\inf _{\boldsymbol{x} \in E} 2 \mu(\boldsymbol{x})$,

$c_{1}^{E}=\sup _{\boldsymbol{x} \in E}(D \lambda(\boldsymbol{x})+2 \mu(\boldsymbol{x}))$.

for the isotropic stiffness tensor.

We obtain the following bounds on the penalty parameter:

Theorem 4.1. (Coercivity) Let $\boldsymbol{X}_{E}$ be an affine function (i.e. the Jacobian is constant). Moreover, let

$\delta_{e}^{*}(\zeta)=\left\{\begin{array}{rll}\frac{D+1}{4 \zeta}\left(\beta_{e, 1}+\beta_{e, 2}\right) & \text { if } & e \in \Gamma^{i}, \\ \frac{(D+1)}{\zeta} \beta_{e, 1} & \text { if } & e \in \Gamma^{D},\end{array}\right.$

where $0<\zeta<1$ and

$\beta_{e, i}=\frac{N(N-1+D)}{D} \frac{|e|}{\left|E_{i}^{e}\right|} \frac{\left(c_{1}^{E_{i}^{e}}\right)^{2}}{c_{0}^{E_{i}^{e}}}$.

Then choosing $\delta_{e}>\delta_{e}^{*}$ ensures

$a(\boldsymbol{u}, \boldsymbol{u}) \geq k^{*}(\zeta)\|\boldsymbol{u}\|_{*}^{2}$

with coercivity constant

$k^{*}(\zeta)=\min \left\{1-\zeta, \min _{e \in \Gamma^{i} \cup \Gamma^{D}}\left(1-\frac{\delta_{e}^{*}(\zeta)}{\delta_{e}}\right)\right\}$.

Proof. See Appendix A.

Corollary 4.1. (Boundedness)

$a(\boldsymbol{u}, \boldsymbol{u}) \leq K^{*}(\zeta)\|\boldsymbol{u}\|_{*}^{2}$

with constant

$K^{*}(\zeta)=\max \left\{1+\zeta, \max _{e \in \Gamma^{i} \cup \Gamma^{D}}\left(1+\frac{\delta_{e}^{*}(\zeta)}{\delta_{e}}\right)\right\}$. 


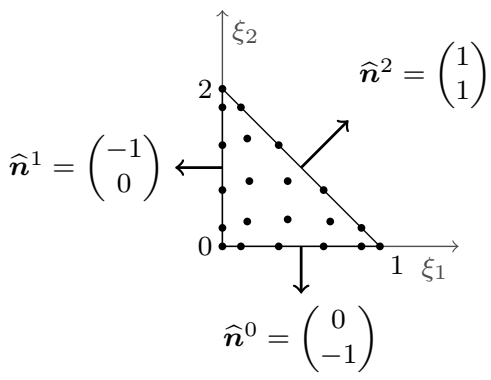

Figure 1. Numbering conventions and normals for the reference triangle. Nodes are plotted for $N=5$, with $N$ being the polynomial degree.

In practice, we do not expect equality to hold in Theorem 4.1. Thus, we set $\delta_{e}$ to its limiting value, i.e. $\delta_{e}=\delta_{e}^{*}(1)$. Moreover, the penalty parameter might also be adequate for curvilinear meshes with little distortion although a bound of the penalty parameter for the general case is desirable. The proof in Appendix A could be extended by establishing one of the following inequalities:

(i) Prove the trace inequality $\|\varepsilon(\boldsymbol{u})\|_{e} \leq C\|\varepsilon(\boldsymbol{u})\|_{E}$ and determine the constant $C$, where we note that $\boldsymbol{\varepsilon}(\boldsymbol{u})$ is non-polynomial.

(ii) Prove the "discrete Korn's inequality" (cf. Brenner (2004)) for curved elements and determine the constant in the inequality.

\section{DISCONTINUOUS GALERKIN IMPLEMENTATION WITH CURVILINEAR COORDINATES AND SUB-CELL MATERIAL RESOLUTION}

The solution to the variational problem (23) is obtained by solving the linear system of equations $\boldsymbol{A} \underline{\boldsymbol{u}}=\boldsymbol{b}$ with

$$
A_{(k m E)(l n F)}=a\left(\phi_{k}^{E} \boldsymbol{e}_{m}, \phi_{l}^{F} \boldsymbol{e}_{n}\right), \quad b_{(k m E)}=L\left(\phi_{k}^{E} \boldsymbol{e}_{m}\right),
$$

where $\left(\boldsymbol{e}_{m}\right)_{i}=\delta_{m i}$ and $\left\{\phi_{k}^{E} \boldsymbol{e}_{m}\right\}_{k, m, E}$ is a basis of $V_{h}$ with $k=1, \ldots,\left(\begin{array}{c}N+D \\ D\end{array}\right), m=1, \ldots, D$, and $E \in \mathcal{T}_{h}$. The local basis functions $\phi_{l}^{E}$ are non-zero on $E$ and zero otherwise. We note that $\underline{\boldsymbol{u}}$ denotes the degrees of freedom of $\boldsymbol{u} \in V_{h}$. Local degrees of freedom are denoted with superscript $E$, i.e. $(\underline{\boldsymbol{u}})_{(\ln E)}=\underline{u}_{l n}^{E}$, such that $\boldsymbol{u}=\sum_{E} \phi_{l}^{E} \boldsymbol{e}_{n} \underline{u}_{l n}^{E}$ with components $u_{i}=\sum_{E} \phi_{l}^{E} \underline{u}_{l i}^{E}$.

Strategies to solve $\boldsymbol{A} \underline{\boldsymbol{u}}=\boldsymbol{b}$ may require the explicit assembly of the non-zero entities of the matrix $\boldsymbol{A}$, or they may use the socalled matrix-free approach, in which one implements the action of $\boldsymbol{A}$ on the degrees of freedom $\underline{u}$, i.e.

$$
\begin{aligned}
(\boldsymbol{A} \underline{\boldsymbol{u}})_{(k m E)}= & \sum_{F} a\left(\phi_{k}^{E} \boldsymbol{e}_{m}, \phi_{l}^{F} \boldsymbol{e}_{n}\right) \underline{u}_{l n}^{F} \\
& =a\left(\phi_{k}^{E} \boldsymbol{e}_{m}, \sum_{F} \phi_{l}^{F} \boldsymbol{e}_{n} \underline{u}_{l n}^{F}\right)=a\left(\phi_{k}^{E} \boldsymbol{e}_{m}, \boldsymbol{u}\right) .
\end{aligned}
$$

In either case, machinery to evaluate volume and surface integrals on the reference element is required, and we detail the necessary tools to implement the DG scheme on unstructured curvilinear meshes in Section 5.1.

\subsection{Computations on the reference element}

We assume that a map from the reference element $\widehat{E}$ to a physical element $E$ is expressed via the following nodal basis expansion:

$\boldsymbol{X}_{E}(\boldsymbol{\xi})=\sum_{j} \phi_{j}(\boldsymbol{\xi}) \underline{\boldsymbol{X}}_{j}^{E}$.

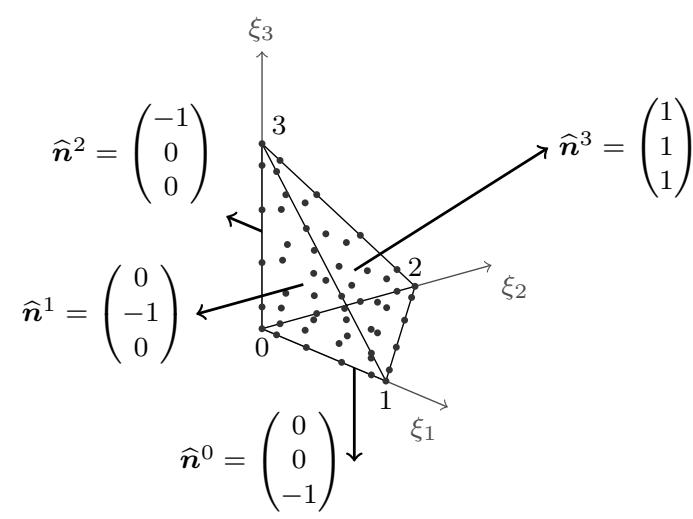

Figure 2. Numbering conventions and normals for the reference tetrahedron. Nodes are plotted for $N=5$.

The defining property of nodal basis functions is $\phi_{j}\left(\boldsymbol{\xi}_{i}\right)=\delta_{i j}$, with Kronecker delta $\delta_{i j}$ and nodes $\boldsymbol{\xi}_{1}, \ldots, \boldsymbol{\xi}_{B_{N}} \in \widehat{E}$, where $B_{N}=\left(\begin{array}{c}N+D \\ D\end{array}\right)$. We use the warp \& blend nodes in this paper as they have a small Lebesgue constant and are easy to construct on the triangle and the tetrahedron (Warburton 2006). The warp \& blend nodes are illustrated in Figures 1 and 2.

The points $\underline{\boldsymbol{X}}_{j}^{E} \in \mathbb{R}^{D}$ give the location of a node in the domain, as $\boldsymbol{X}_{E}\left(\boldsymbol{\xi}_{j}\right)=\underline{\boldsymbol{X}}_{j}^{E}$. In practice, these can be obtained in at least two ways: Either, one generates a simplex mesh and applies a warp function to each node, such that $\boldsymbol{X}_{E}$ is an interpolant of the warp function. Or, one can use high-order meshing software, e.g. the open-source software Gmsh (Geuzaine \& Remacle 2009; Johnen et al. 2013).

Volume integrals over the physical element $E=\boldsymbol{X}_{E}(\widehat{E})$ can be computed using quadrature rules (Witherden \& Vincent 2015; Jaśkowiec \& Sukumar 2020). For example, the integral of $\boldsymbol{u} \in V_{h}$ is computed with

$$
\begin{aligned}
\int_{E} u_{p}(\boldsymbol{x}) \mathrm{d} \boldsymbol{x}=\int_{\widehat{E}} \underline{u}_{i p}^{E} \phi_{i}(\boldsymbol{\xi}) J^{E}(\boldsymbol{\xi}) \mathrm{d} \boldsymbol{\xi} & \\
& \approx \sum_{q} W_{q} \underline{u}_{i p}^{E} \phi_{i}\left(\widehat{\boldsymbol{\xi}}_{\boldsymbol{q}}\right) J^{E}\left(\widehat{\boldsymbol{\xi}}_{q}\right),
\end{aligned}
$$

where we use the nodal basis expansion $\left.u_{p}\right|_{E} \circ \boldsymbol{X}_{E}=\underline{u}_{i p}^{E} \phi_{i}(\boldsymbol{\xi})$, $W_{q}$ and $\widehat{\boldsymbol{\xi}}_{q}$ are quadrature weights and abscissa for $\widehat{E}$, and

$J^{E}(\boldsymbol{\xi})=\left|\operatorname{det}\left(\nabla \boldsymbol{X}_{E}(\boldsymbol{\xi})\right)\right|$.

For the surface integral's parameterisation, we introduce the maps $\Upsilon_{E}^{f}: \widehat{e} \rightarrow \widehat{E}$ from the reference facet $\widehat{e}$ (i.e. reference edge in $2 \mathrm{D}$ and reference triangle in $3 \mathrm{D}$ ) to the reference element $\widehat{E}$, given by

$\mathbf{\Upsilon}^{f}(\boldsymbol{\chi})=\left(1-\sum_{i=1}^{D-1} \chi_{i}\right) \boldsymbol{\xi}_{0}^{f}+\sum_{i=1}^{D-1} \chi_{i} \boldsymbol{\xi}_{i}^{f}$,

where $\boldsymbol{\xi}_{0}^{f}, \ldots, \boldsymbol{\xi}_{D-1}^{f}$ are the vertices of the reference simplex that make up the $f$-th facet $(f=0, \ldots, D)$.

Surface integrals in DG involve information of the two simplices $E_{1}$ and $E_{2}$ adjoining the facet $e=\partial E_{1} \cap \partial E_{2}$. Let $f, g$ be the local face number of $e$ in $E_{1}, E_{2}$, respectively, and $\Upsilon^{f}$ and $\mathbf{\Upsilon}^{g}$ the respective facet maps. The parameterisation is greatly simplified by ensuring consistent local edge directions. For triangle or tetrahedral meshes, one can show that local edge directions are always consistent when the local vertex order is chosen according to 
a global vertex order (Rognes et al. 2009). In this case, we have

$\forall \chi \in \widehat{e}: \boldsymbol{X}_{E_{1}} \circ \boldsymbol{\Upsilon}^{f}=\boldsymbol{X}_{E_{2}} \circ \boldsymbol{\Upsilon}^{g}$.

For example, for $\boldsymbol{u}, \boldsymbol{v} \in V_{h}$ we have

$$
\begin{gathered}
\left.\left.\int_{e} u_{p}\right|_{E_{1}} v_{p}\right|_{E_{2}} \mathrm{~d} s=\int_{\widehat{e}} \underline{u}_{i p}^{E_{1}} \phi_{i}\left(\mathbf{\Upsilon}^{f}(\boldsymbol{\chi})\right) \underline{v}_{j p}^{E_{2}} \phi_{j}\left(\mathbf{\Upsilon}^{g}(\boldsymbol{\chi})\right)\left|\boldsymbol{n}^{e}(\boldsymbol{\chi})\right| \mathrm{d} \boldsymbol{\chi} \\
\approx \sum_{q} w_{q} \underline{u}_{i p}^{E_{1}} \phi_{i}\left(\mathbf{\Upsilon}^{f}\left(\widehat{\chi}_{\boldsymbol{q}}\right)\right) \underline{v}_{j p}^{E_{2}} \phi_{j}\left(\boldsymbol{\Upsilon}^{g}\left(\widehat{\chi}_{\boldsymbol{q}}\right)\right)\left|\boldsymbol{n}^{e}\left(\widehat{\boldsymbol{\chi}}_{\boldsymbol{q}}\right)\right|
\end{gathered}
$$

where we have used the nodal basis expansions $\left.u_{p}\right|_{E_{1}} \circ \boldsymbol{X}_{E_{1}}=$ $\underline{u}_{i p}^{E_{1}} \phi_{i}(\boldsymbol{\xi})$ and $\left.v_{p}\right|_{E_{2}} \circ \boldsymbol{X}_{E_{2}}=\underline{v}_{i p}^{E_{2}} \phi_{i}(\boldsymbol{\xi})$. Quadrature weights and abscissa for $\widehat{e}$ are given by $w_{q}$ and $\widehat{\chi}_{\boldsymbol{q}}$ (Witherden \& Vincent 2015) and

$\boldsymbol{n}^{e}(\boldsymbol{\chi})=J^{E_{1}}\left(\boldsymbol{\Upsilon}^{f}(\boldsymbol{\chi})\right)\left(\boldsymbol{\nabla} \boldsymbol{X}_{E_{1}}\left(\boldsymbol{\Upsilon}^{f}(\boldsymbol{\chi})\right)\right)^{-T} \widehat{\boldsymbol{n}}^{f}$,

where $\widehat{\boldsymbol{n}}^{f}$ are the outward pointing normals of the reference simplex (cf. Figures 1 and 2). We remark that the absolute value of the determinant of the face Jacobian is taken in (36), because the determinant might be negative as a consequence of the numbering convention. (E.g. in 2D the vertices might be ordered both clockwise and counter-clockwise.) The same reasoning also holds for the cell Jacobian in (32).

\subsection{Residual evaluation and matrix-free implementation}

We first eliminate the derivative in (17) through integration by parts:

$$
\begin{aligned}
\int_{E} \tau_{i j} \sigma_{i j} \mathrm{~d} \boldsymbol{x}=\int_{E} \tau_{i j} c_{i j r s} & \frac{\partial u_{r}}{\partial x_{s}} \mathrm{~d} \boldsymbol{x} \\
& +\int_{\partial E} \tau_{i j} c_{i j r s}\left(\widetilde{u}_{r}-u_{r}\right) n_{s} \mathrm{~d} s .
\end{aligned}
$$

We set $\tau_{i j}=\partial v_{i} / \partial x_{j}$ in (37), which is valid due to $\nabla_{h} V_{h} \subset \Sigma_{h}$. The result is plugged into (18):

$$
\begin{aligned}
\int_{E} \frac{\partial v_{i}}{\partial x_{j}} c_{i j r s} \frac{\partial u_{r}}{\partial x_{s}} \mathrm{~d} \boldsymbol{x}+ & \int_{\partial E} \frac{\partial v_{i}}{\partial x_{j}} c_{i j r s}\left(\widetilde{u}_{r}-u_{r}\right) n_{s} \mathrm{~d} s \\
& -\int_{\partial E} v_{i} \widetilde{\sigma}_{i j} n_{j} \mathrm{~d} s=\int_{E} f_{i} v_{i} \mathrm{~d} \boldsymbol{x} .
\end{aligned}
$$

The computation of the numerical flux on $e=\partial E_{1}^{e} \cap \partial E_{2}^{e}$ is described next. Recall the basis expansion $\boldsymbol{u}=\sum_{E} \phi_{l}^{E} \boldsymbol{e}_{n} \underline{u}_{l n}^{E}$, where $\phi_{l}^{E}$ is only non-zero on $E$. If $e \in \Gamma^{0}$ we have for $\boldsymbol{x} \in e$

$$
\begin{aligned}
\widetilde{\sigma}_{i j}^{e}(\boldsymbol{x}) & =\left\{\left\{c_{i j r s} \frac{\partial u_{r}}{\partial x_{s}}\right\}\right\}-\delta_{e} \llbracket u_{i} \rrbracket n_{j}=\frac{1}{2} c_{i j r s}^{E_{1}^{e}} \frac{\partial \phi_{l}^{E_{1}^{e}}}{\partial x_{s}} \underline{u}_{l r}^{E_{1}^{e}} \\
& +\frac{1}{2} c_{i j r s}^{E_{2}^{e}} \frac{\partial \phi_{l}^{E_{2}^{e}}}{\partial x_{s}} \underline{u}_{l r}^{E_{2}^{e}}-\delta_{e}\left(\underline{u}_{l i}^{E_{1}^{e}} \phi_{l}^{E_{1}^{e}}-\underline{u}_{l i}^{E_{2}^{e}} \phi_{l}^{E_{2}^{e}}\right) n_{j} .
\end{aligned}
$$

For $\boldsymbol{x} \in E$ the basis functions are given by $\phi_{l}^{E}=\phi_{l} \circ \boldsymbol{X}_{E}^{-1}$. Using the chain rule we obtain (Brezzi \& Fortin 1991, III.1.3)

$\boldsymbol{\nabla} \phi_{k}^{E}=\left(\boldsymbol{\nabla} \phi_{l} \circ \boldsymbol{X}_{E}^{-1}\right) \boldsymbol{\nabla} \boldsymbol{X}_{E}^{-1}=\left(\boldsymbol{\nabla} \phi_{l}\left(\boldsymbol{\nabla} \boldsymbol{X}_{E}\right)^{-1}\right) \circ \boldsymbol{X}_{E}^{-1}$.

Moreover, we introduce the shorthand notation

$$
\begin{array}{rlrl}
G_{e j}^{E}(\boldsymbol{\xi}) & =\left(\boldsymbol{\nabla} \boldsymbol{X}_{E}(\boldsymbol{\xi})\right)_{e j}^{-1}, & D_{k e}(\boldsymbol{\xi})=\frac{\partial \phi_{k}(\boldsymbol{\xi})}{\partial \xi_{e}}, \\
G_{e j}^{E, f}(\boldsymbol{\chi}) & =G_{e j}^{E}\left(\mathbf{\Upsilon}^{f}(\boldsymbol{\chi})\right), \quad D_{k e}^{f}(\boldsymbol{\chi})=D_{k e}\left(\mathbf{\Upsilon}^{f}(\boldsymbol{\chi})\right), \\
\phi_{k}^{f}(\boldsymbol{\chi}) & =\phi_{k}\left(\boldsymbol{\Upsilon}^{f}(\boldsymbol{\chi})\right),
\end{array}
$$

where $f=0, \ldots, D$ is the local facet number. Let $f_{1}^{e}$ and $f_{2}^{e}$ be the local facet numbers for $e$ of elements $E_{1}^{e}$ and $E_{2}^{e}$, respectively. Hence, we get on the reference facet

$$
\begin{aligned}
\tilde{\sigma}_{i j}^{e}(\chi)=\frac{1}{2} c_{i j r s}^{E_{1}^{e}} D_{l e}^{f_{1}^{e}} G_{e s}^{E_{1}^{e}} f_{1}^{e} & \underline{u}_{l r}^{E_{1}^{e}}+\frac{1}{2} c_{i j r s}^{E_{2}^{e}} D_{l e}^{f_{2}^{e}} G_{e s}^{E_{2}^{e}, f_{2}^{e}} \underline{u}_{l r}^{E_{2}^{e}} \\
& -\delta_{e}\left(\underline{u}_{l i}^{E_{1}^{e}} \phi_{l}^{f_{1}^{e}}-\underline{u}_{l i}^{E_{2}^{e}} \phi_{l}^{f_{2}^{e}}\right) n_{j}^{e} .
\end{aligned}
$$

Likewise, the displacement flux on the reference facet is

$\widetilde{u}_{i}^{e}(\chi)=\left\{\left\{u_{i}\right\}=\frac{1}{2}\left(\underline{u}_{l i}^{E_{1}^{e}} \phi_{l}^{f_{1}^{e}}+\underline{u}_{l i}^{E_{2}^{e}} \phi_{l}^{f_{2}^{e}}\right)\right.$.

The numerical fluxes on the fault, Dirichlet, or Neumann boundaries are derived similarly. We only note that on $e \in \Gamma^{F}$ the flux is double valued with $\left.\widetilde{u}_{i}\right|_{E_{1}^{e}}=\left\{\left\{u_{i}\right\}\right\}+g_{i}^{F} / 2$ and $\left.\widetilde{u}_{i}\right|_{E_{2}^{e}}=$ $\left\{\left\{u_{i}\right\}-g_{i}^{F} / 2\right.$.

Next, we recover an expression for the residual $\boldsymbol{r}:=\boldsymbol{A} \underline{\boldsymbol{u}}-\boldsymbol{b}$. In (38), we move the force term to the left-hand side and plug in the basis expansion and the test function $\boldsymbol{v}=\boldsymbol{e}_{u} \phi_{k}^{E}$ :

$$
\begin{gathered}
r_{(k u E)}=\underline{u}_{l r}^{E} \int_{E} \frac{\partial \phi_{k}^{E}}{\partial x_{j}} c_{u j r s} \frac{\partial \phi_{l}^{E}}{\partial x_{s}} \mathrm{~d} \boldsymbol{x} \\
+\sum_{e \in \partial E} \int_{e} \frac{\partial \phi_{k}^{E}}{\partial x_{j}} c_{u j r s}\left(\widetilde{u}_{r}^{e}-\underline{u}_{l r}^{E} \phi_{l}^{E}\right) n_{s} \mathrm{~d} s \\
\quad-\sum_{e \in \partial E} \int_{e} \phi_{k}^{E} \widetilde{\sigma}_{u j}^{e} n_{j} \mathrm{~d} s-\int_{E} \phi_{k}^{E} f_{u} \mathrm{~d} \boldsymbol{x} .
\end{gathered}
$$

With the tools developed in Section 5.1 we map (44) to the reference element:

$$
\begin{gathered}
r_{(k u E)}=\underline{u}_{l r}^{E} \int_{\widehat{E}} D_{k e} G_{e j}^{E} c_{u j r s} D_{l f} G_{f s}^{E} J^{E} \mathrm{~d} \boldsymbol{\xi} \\
+\sum_{f=0}^{D} \int_{\widehat{e}} D_{k e}^{f} G_{e j}^{E, f} c_{u j r s}\left(\widetilde{u}_{r}^{e_{f}}-\underline{u}_{l r}^{E} \phi_{l}^{f}\right) n_{s}^{e_{f}} \mathrm{~d} \boldsymbol{\chi} \\
\quad-\sum_{f=0}^{D} \int_{\widehat{e}} \phi_{k}^{f} \widetilde{\sigma}_{u j}^{e_{f}} n_{j}^{e_{f}} \mathrm{~d} \boldsymbol{\chi}-\int_{\widehat{E}} \phi_{k} f_{u} J^{E} \mathrm{~d} \boldsymbol{\xi},
\end{gathered}
$$

where $e_{f}$ is the $f$-th facet of $E$. Finally, we introduce the convention that an additional index $q$ means evaluation at $\widehat{\boldsymbol{\xi}}_{q}$ or $\widehat{\boldsymbol{\chi}}_{q}$, e.g. $G_{e j q}^{E}:=G_{e j}^{E}\left(\widehat{\boldsymbol{\xi}}_{\boldsymbol{q}}\right)$ or $n_{i q}^{e}:=n_{i}^{e}\left(\widehat{\boldsymbol{\chi}}_{\boldsymbol{q}}\right)$. The fully discrete form is

$$
\begin{aligned}
& r_{(k u E)} \approx \sum_{q} W_{q} \underline{u}_{l r}^{E} D_{k e q} G_{e j q}^{E} c_{u j r s q} D_{l f q} G_{f s q}^{E} J_{q}^{E} \\
& +\sum_{f} \sum_{q} w_{q} D_{k e q}^{f} G_{e j q}^{E, f} c_{u j r s q}\left(\widetilde{u}_{r q}^{e_{f}}-\underline{u}_{l r}^{E} \phi_{l q}^{f}\right) n_{s q}^{e_{f}} \\
& \quad-\sum_{f} \sum_{q} w_{q} \phi_{k q}^{f} \widetilde{\sigma}_{u j q}^{e_{f}} n_{j q}^{e_{f}}-\sum_{q} W_{q} \phi_{k q} f_{u q} J_{q}^{E} .
\end{aligned}
$$

The action $\boldsymbol{A} \underline{\boldsymbol{u}}$ is readily obtained by setting $g_{i}^{D}=0, g_{i}^{F}=0$, and $f_{i}=0$ in the residual. That is, setting all terms which would appear in $\boldsymbol{b}$ (in the discrete problem), or in $L(\boldsymbol{v})$ (in the continuous problem), to zero.

\subsection{Linear solvers}

There are many direct and iterative methods to find the solution of the sparse linear system $\boldsymbol{A} \underline{\boldsymbol{u}}=\boldsymbol{b}$. We gain access to many of these methods by using the Portable, Extensible Toolkit for Scientific Computation (PETSc), which provides high-level data structures for distributed matrices and vectors and a unified interface to many Krylov methods, preconditioners, and sparse direct solvers (Balay et al. 1997, 2021a,b). 
In this paper, we use two different kinds of solvers, $\mathrm{LU}$ factorisation and Krylov methods preconditioned with multigrid. LU factorisation is a good choice for small-scale problems, in particular in 2D. For SEAS problems, the operator $\boldsymbol{A}$ is time-invariant, such that the expensive factorization step is only required once. PETSc provides an interface to many state-of-the-art LU factorisation packages (Balay et al. 2021a). In this work, when we resort to using LU, we used the sparse direct solver MUMPS (Amestoy et al. 2001, 2019).

For large problems, in particular in 3D, iterative methods are used with a preconditioner that combines the p-multigrid and the algebraic multigrid methods. We define a sequence of coarse to fine grids $V_{h}^{p_{1}} \subset \cdots \subset V_{h}^{p_{L}}$ for the p-multigrid method, where $p_{1}=1<\cdots<p_{L}=N$. The spaces $V_{h}^{p_{i}}$ are obtained by replacing $N$ with $p_{i}$ in (16). As the spaces are nested and the same mesh is used, the interpolation operator $\boldsymbol{I}_{i}^{i+1}$ for the transfer of the residual from grid $i$ to grid $i+1$ is simply a block diagonal matrix. Following the Galerkin approach, the restriction operator is set to $\boldsymbol{I}_{i+1}^{i}=\left(\boldsymbol{I}_{i}^{i+1}\right)^{T}$ and the coarse grid operators are given by $\boldsymbol{A}^{i}=\boldsymbol{I}_{i+1}^{i} \boldsymbol{A}^{i+1} \boldsymbol{I}_{i}^{i+1}$. On the coarsest grid, we apply the algebraic multigrid preconditioner GAMG (Balay et al. 2021a).

\subsection{Implementation details}

Our C++-implementation of the DG scheme is freely available under the BSD 3-Clause license (https://github.com/ TEAR-ERC/tandem).

Tandem supports matrix assembly as well as the matrix-free approach. The matrix-free computation of the residual or operator application $\boldsymbol{A} \underline{\boldsymbol{u}}$ consists solely of small tensor contractions. We use Yet Another Tensor Toolbox (Uphoff \& Bader 2020) to generate high-performance kernels for (46).

Tandem is inherently parallel and designed for large scale distributed memory architectures. In addition to PETSc for parallel linear algebra, solvers, and preconditioner support, we also employ the graph partitioning libraries Metis and Parmetis (Karypis \& Kumar 1998) to facilitate run-time mesh partitioning for a given MPI communicator size.

Tandem natively loads meshes generated with Gmsh in parallel. Once loaded, a new mesh decomposition (load balanced partition) is defined using Parmetis. Given the new partition, the elements and all associated attributes (e.g. cell tags / labels, vertex coordinates, vertex tags / labels) are scattered to the appropriate MPI rank. In this paper, all meshes were created using Gmsh version 4.8.4 (Geuzaine \& Remacle 2009; Johnen et al. 2013).

In principle, given a DG function space of degree $N$, the error is expected to decrease with $\mathcal{O}\left(h^{N}\right)$, where $h$ is the maximum element diameter. In order to retain the desirable convergence behaviour in domains with curved boundary or heterogeneous material parameters, we approximate the geometry and material parameters with polynomials of the same maximum degree $N$ as the displacement.

\section{COUPLING AND TIME-STEPPING}

We complete the numerical scheme for SEAS model with the following four steps. First, we introduce trace spaces on $\Gamma^{F}$ in order to approximate the on-fault traction, slip, and state variable. Second, we discuss the coupling between on-fault and off-fault variables. Third, we show how (12) and (11) can be recast as an ordinary system of differential equations. Fourth, we discuss time-stepping.

\subsection{Trace spaces}

The mapping $\boldsymbol{m}_{e}: \widehat{e} \rightarrow e$ from the reference facet $\widehat{e}$ to $e \in \Gamma^{F}$ is in the notation of Section 5 given by

$\boldsymbol{m}_{e}(\boldsymbol{\chi})=\boldsymbol{X}_{E_{1}^{e}} \circ \boldsymbol{\Upsilon}^{f_{1}^{e}}=\boldsymbol{X}_{E_{2}^{e}} \circ \boldsymbol{\Upsilon}^{f_{2}^{e}}$.

Quantities $(\boldsymbol{T}, \boldsymbol{S}, \psi)$ on the physical element are related to quantities $(\widehat{\boldsymbol{T}}, \widehat{\boldsymbol{S}}, \widehat{\psi})$ on the reference element via

$\boldsymbol{T}=\widehat{\boldsymbol{T}} \circ \boldsymbol{m}_{e}^{-1}, \quad \boldsymbol{S}=\widehat{\boldsymbol{S}} \circ \boldsymbol{m}_{e}^{-1}, \quad \psi=\widehat{\psi} \circ \boldsymbol{m}_{e}^{-1}$

We introduce the finite element spaces

$M_{h}^{d}=\left\{\boldsymbol{\mu} \in\left[L^{2}\left(\Gamma^{F}\right)\right]^{d}: \widehat{\boldsymbol{\mu}}_{e} \in\left[P_{N}(\widehat{e})\right]^{d} \forall e \in \Gamma^{F}\right\}$

for $\boldsymbol{T}, \boldsymbol{S} \in M_{h}^{D-1}$ and $\psi \in M_{h}^{1}$.

\subsection{Coupling}

The change in traction $\delta T_{i}=T_{i}-T_{i}^{0}=B_{j i} \sigma_{j k}(\boldsymbol{u}) n_{k}$ couples the elasticity solver to the friction law, but $\sigma_{j k}$ is double-valued on $\Gamma^{F}$. Thus, we replace with $\sigma_{j k}$ with the numerical flux $\widetilde{\sigma}_{j k}$, because the flux is single-valued and is designed to be a (stable) approximation of $\sigma_{j k}$ on $\Gamma^{F}$.

Moreover, $\left.\left(B^{T} \widetilde{\sigma} \boldsymbol{n}\right)\right|_{\Gamma^{F}} \notin M_{h}$ for non-affine mappings. Hence, we define the projection operator $\pi_{e}^{d}:\left[L^{2}\left(\Gamma^{F}\right)\right]^{d} \rightarrow M_{h}^{d}$,

$\forall \boldsymbol{\mu} \in M_{h}^{d}: \int_{e} \boldsymbol{\mu} \cdot \pi_{e}(\boldsymbol{\theta}) \mathrm{d} s=\int_{e} \boldsymbol{\mu} \cdot \boldsymbol{\theta} \mathrm{d} s$,

and let $\left.\boldsymbol{\delta} \boldsymbol{T}\right|_{e}=\pi_{e}^{D-1}\left(B^{T} \widetilde{\sigma} \boldsymbol{n}\right)$. In the implementation, we represent the change in traction using the nodal basis $\varphi_{i}(\chi)$ on the reference face $\widehat{e}$. I.e. with $\left.\delta T_{i}\right|_{e} \circ \boldsymbol{m}_{e}=\sum_{l} \underline{\delta T}_{l i}^{e} \varphi_{l}$ we have

$\underline{\delta T}_{l i}^{e} \int_{\widehat{e}} \varphi_{k} \varphi_{l}\left|\boldsymbol{n}^{e}\right| \mathrm{d} \boldsymbol{\chi}=\int_{\widehat{e}} \varphi_{k} B_{j i}^{e} \widetilde{\sigma}_{i j}^{e} n_{j}^{e} \mathrm{~d} \boldsymbol{\chi}$.

Likewise, the change in normal stress is with $\delta \sigma_{n}=\pi_{e}^{1}(\boldsymbol{n} \tilde{\sigma} \boldsymbol{n})$.

Slip is set as boundary condition in the elasticity solver, cf. (12). Using the nodal basis expansion $\widehat{S}_{j}=\sum_{l} \underline{S}_{l j}^{e} \varphi_{l}(\chi)$ we simply set $\left.g_{i}^{F}\right|_{e} \circ \boldsymbol{m}_{e}=B_{i j}^{e} \underline{S}_{l j}^{e} \varphi_{l}$.

We note that in an early stage of developing the numerical scheme we defined on-fault traction as $\left\{\left\{c_{i j k l} \partial u_{k} / \partial x_{l}\right\}\right\} n_{j}$ instead of $\widetilde{\sigma}_{i j} n_{j}$. We found that this choice is unstable, i.e. the onfault traction might become discontinuous along the fault and grow without bounds.

\subsection{Ordinary differential equations}

The Dirichlet and fault boundary conditions only appear in the right-hand side $L(\boldsymbol{v})$, cf. (22). Hence, the discrete elasticity problem in dependence on $\operatorname{slip} \boldsymbol{S}$ and time $t$ is given by

$\boldsymbol{A} \underline{\boldsymbol{u}}=\boldsymbol{b}(\underline{\boldsymbol{S}}, t)$

The above problem has a unique solution for a large enough penalty (cf. Section 4) formally given by $\underline{\boldsymbol{u}}=\boldsymbol{A}^{-1} \boldsymbol{b}(\underline{\boldsymbol{S}}, t)$. The change $\boldsymbol{\delta} \boldsymbol{T}$ in traction depends linearly on $\underline{\boldsymbol{u}}$, cf. Section 6.2 , thus traction $\boldsymbol{T}$ is a function of time and slip.

Slip-rate, state, and traction are linked via the friction law. Taking the magnitude $T=|\boldsymbol{T}|$ reduces (10) to the single equation

$\sigma_{n} F(V, \psi)+\eta V-T=0$,

where $V=|\boldsymbol{V}|$. The derivative of (53) w.r.t. $V$ is $\sigma_{n} \frac{\partial F}{\partial V}+\eta$, where for the particular choice of $F$ from (6) we have $\partial F / \partial V>0$ 


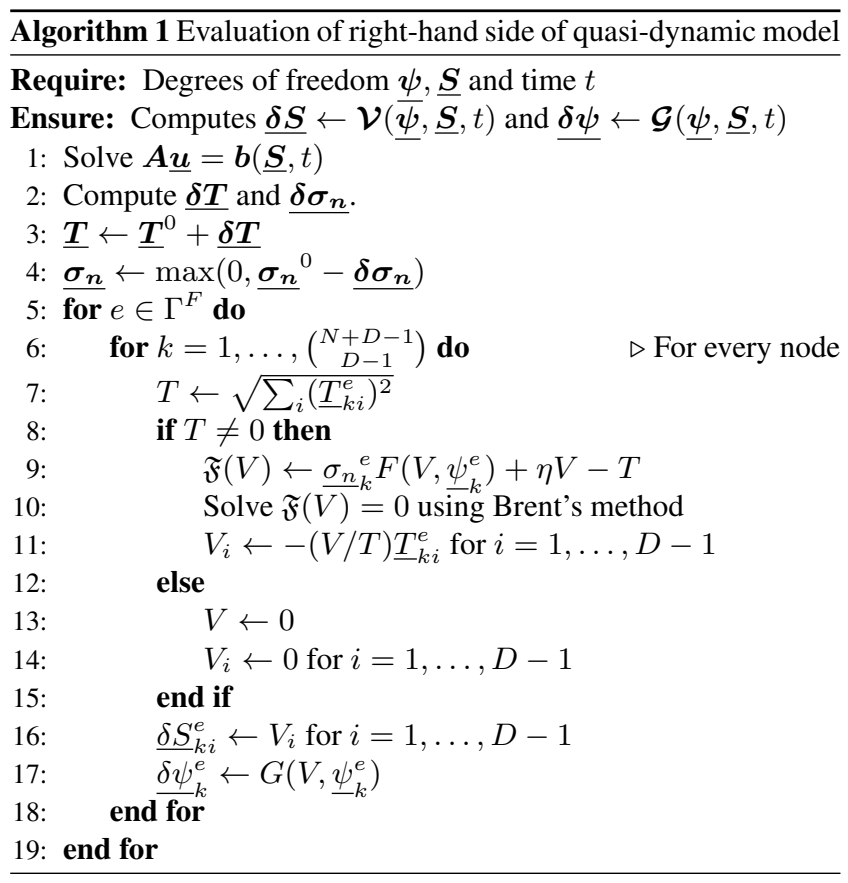

for $a>0$ and $V_{0}>0$. With $\eta \geq 0$ it follows from the implicit function theorem that there exists a unique function $V\left(\psi, T, \sigma_{n}\right)$ that solves (53). It follows that $\boldsymbol{V}$ is a function of $\psi, \boldsymbol{T}, \sigma_{n}$.

In practice, the solution to (53) is found using standard rootfinding methods such as Brent's method (Forsythe et al. 1977). Note that the root lies in the interval $V \in[0, T / \eta]$.

So far we silently ignored that $\boldsymbol{S}, \psi$ depend on time. With abuse of notation we understand that for fixed time $t$ we have $\boldsymbol{S}(t) \in M_{h}^{D-1}$ and $\psi(t) \in M_{h}^{1}$. We solve for slip and state on every node, i.e. the semi-discrete quasi-dynamic model is

$$
\begin{aligned}
\frac{\mathrm{d} \underline{\boldsymbol{S}}}{\mathrm{d} t} & =\mathcal{V}(\underline{\boldsymbol{\psi}}, \underline{\boldsymbol{S}}, t), \\
\frac{\mathrm{d} \underline{\boldsymbol{\psi}}}{\mathrm{d} t} & =\mathcal{G}(\underline{\boldsymbol{\psi}}, \underline{\boldsymbol{S}}, t),
\end{aligned}
$$

where we show how to evaluate $\mathcal{V}$ and $\mathcal{G}$ in Algorithm 1 .

\subsection{Time-stepping}

Equation (54) is the canonical formulation of a system of ordinary differential equations. Hence, time-stepping is handled by the software library PETSc/TS (Abhyankar et al. 2018). Experiments in this paper use explicit Runge-Kutta schemes with adaptive timestep control.

\section{DISCRETE GREEN'S FUNCTION}

The most expensive part in Algorithm 1 is the solution of the discrete elasticity problem in Line 1 . Given that SEAS problems typically require hundreds of thousands of time-steps, we may need to solve the discrete elasticity problem millions of times, one solve per Runge-Kutta stage. Moreover, we solve for displacement in a $D$ dimensional domain whereas only the on-fault traction on $(D-1)$ dimensional surfaces is required to evolve rate and state friction. In the following we denote the number of displacement components in the volume by $N_{h}$, and the number of slip components on the fault by $n_{h}$.
By inspecting the linear form $L(\boldsymbol{v})$ in (23), and assuming zero body forces as well as a linear in time Dirichlet boundary condition, we can split up the discrete right-hand side as

$\boldsymbol{b}(\underline{\boldsymbol{S}}, t)=t \boldsymbol{b}_{D}+\boldsymbol{Z}_{1} \underline{\boldsymbol{S}}$,

where the size of $\boldsymbol{b}$ and $\boldsymbol{b}_{D}$ is $N_{h}$, the size of $\underline{\boldsymbol{S}}$ is $n_{h}$, and $\boldsymbol{Z}_{1}$ is a $N_{h} \times n_{h}$ matrix. Moreover, the linear relation between change in traction and displacement is written as

$\underline{\delta T}=Z_{2} \underline{u}$,

where $\boldsymbol{Z}_{2}$ is a $n_{h} \times N_{h}$ matrix. With $\underline{\boldsymbol{S}}=\underline{S}_{i} \boldsymbol{e}_{i},\left(\boldsymbol{e}_{i}\right)_{j}=\delta_{i j}$, we obtain

$\underline{\boldsymbol{\delta} \boldsymbol{T}}=t \underbrace{\boldsymbol{Z}_{2} \boldsymbol{A}^{-1} \boldsymbol{b}_{D}}_{=: \boldsymbol{g}_{D}}+\sum_{i=1}^{n_{h}} \underbrace{\boldsymbol{Z}_{2} \boldsymbol{A}^{-1} \boldsymbol{Z}_{1} \boldsymbol{e}_{i}}_{=: \boldsymbol{g}_{i}} \underline{S}_{i}$.

Therefore, instead of solving a linear system the change in traction can be written in terms of the discrete Green's function $\boldsymbol{g}_{D}, \boldsymbol{g}_{1}, \ldots, \boldsymbol{g}_{n_{h}}$.

The matrices $\boldsymbol{A}^{-1}, \boldsymbol{Z}_{1}, \boldsymbol{Z}_{2}$ are not required explicitly. Instead, we only need to compute the change in traction for the right-hand sides $\boldsymbol{b}(\mathbf{0}, 1)$ and $\boldsymbol{b}\left(\boldsymbol{e}_{i}, 0\right), i=1, \ldots, n_{h}$, in order to obtain the discrete Green's function. We also note that the action of $\boldsymbol{A}^{-1} \boldsymbol{y}$ (for an arbitrary vector $\boldsymbol{y}$ ) is defined via a solve. As an example we consider the first term on the right-hand side of Equation (57). This would be evaluated as $t \boldsymbol{Z}_{2} \boldsymbol{u}^{*}$ where $\boldsymbol{u}^{*}$ is the solution to $\boldsymbol{A} \boldsymbol{u}^{*}=\boldsymbol{b}_{D}$. The solution strategies employed in Section 5.3 are also adopted for obtaining the auxiliary solutions required to define the discrete Green's function. Since the operator $\boldsymbol{A}$ is sparse and we have both optimal and parallel efficient solvers for this operator, we thus also have a highly scalable means to compute all of the required Green's functions. The importance of this will be further elaborated upon in Section 10.2.

\section{VERIFICATION}

The presented SIPG method allows to model the slip boundary condition, curved boundaries, heterogeneous material parameters, and can be coupled to rate and state friction laws. In this section, we discuss and analyse various 2D and 3D model problems whereby each verifies one or or multiple of these method capabilities.

First, we discuss static elasticity problems which verify that the SIPG method converges. An analytic solution is available for each of these problems, hence we determine the empirical convergence order and discuss the benefits and limitations of using a highorder method.

Second, we use the method of manufactured solutions to analyse whether convergence is achieved for a SEAS problem with a modified state equation. Due to manufacturing, the analytic solution is available such that the convergence behaviour of the method can be discussed.

Last, we verify the method in a community benchmark problem Erickson et al. (2020). While this problems resembles an actual SEAS model, no analytic solution is available, but we obtain a comparison to several independent methods and implementations.

We note that input files to reproduce all setups are openly available (see DATA AVAILABILITY).

\subsection{Convergence of the elasticity solver}

We analyse the convergence behaviour of the SIPG scheme for elastostatic problems. In our chosen verification problems we cover 


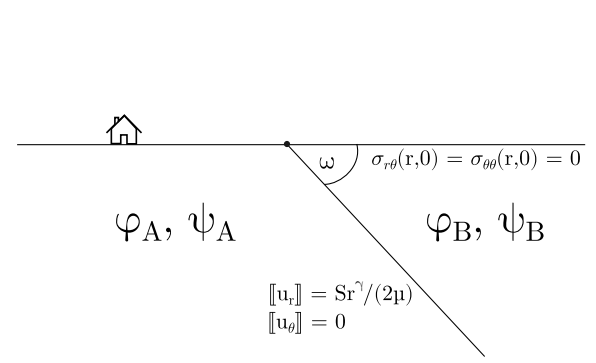

(a) Verification setup of the slip boundary condition.
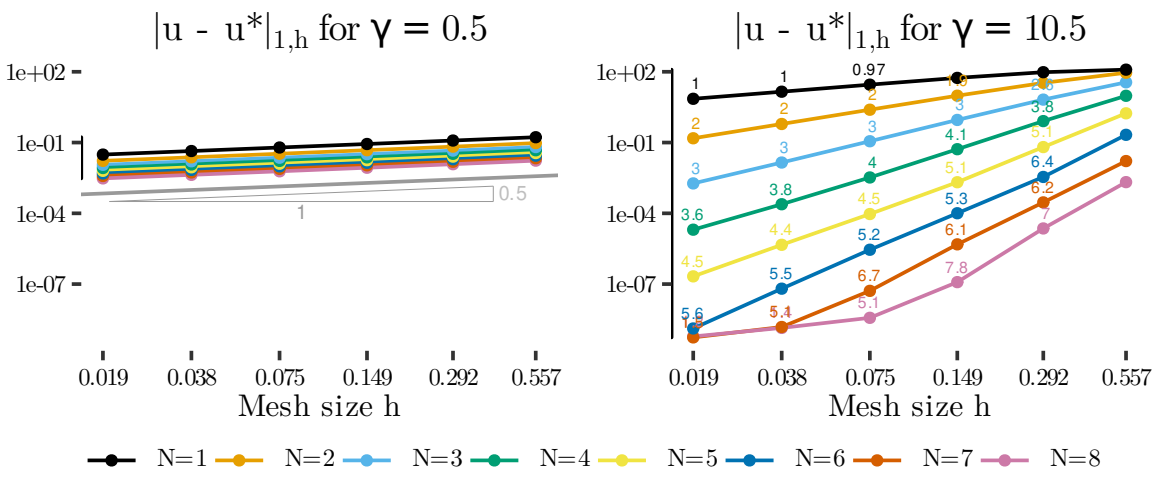

(b) Error vs. mesh size for a solution with poor regularity (left) and a smooth solution (right).

Figure 3. We show the setup and numerical results for a dipping fault scenario which verifies the slip boundary condition. Our results agree well with the theoretical predictions, that is, an empirical convergence order smaller than 1 for $\gamma=0.5$ and empirical convergence orders close to $N$ for $\gamma=10.5$ (cf. small text above points), with $N$ being the polynomial degree and $\gamma$ a solution smoothness parameter. The minimum attainable error is limited due to finite precision and conditioning of the linear system, see text.

two- and three-dimensional curvilinear meshes with heterogeneous material parameters as well as the slip boundary condition.

Errors in the displacement are measured with the norm

$\|\boldsymbol{u}\|_{0, h}=\left(\sum_{E \in \mathcal{T}_{h}} \int_{E} u_{i} u_{i} \mathrm{~d} \boldsymbol{x}\right)^{1 / 2}$.

When coupling the elasticity solver to the non-linear rate and state friction law we need the Jacobian of the displacement. Therefore, we also measure the error in the semi-norm

$|\boldsymbol{u}|_{1, h}=\left(\sum_{E \in \mathcal{T}_{h}} \int_{E} \frac{\partial u_{i}}{\partial x_{j}} \frac{\partial u_{i}}{\partial x_{j}} \mathrm{~d} \boldsymbol{x}\right)^{1 / 2}$

Given a sequence of meshes with mesh sizes $h_{1}>\cdots>h_{n}$, respective discrete solutions $\boldsymbol{u}_{1}, \ldots, \boldsymbol{u}_{n}$, and the analytical solution $\boldsymbol{u}^{*}$, the empirical convergence order is defined as

$\mathcal{C}_{i}=\frac{\log \left(E_{i-1} / E_{i}\right)}{\log \left(h_{i-1} / h_{i}\right)}$,

where the error $E_{i}$ is either given by $\left\|\boldsymbol{u}_{i}-\boldsymbol{u}^{*}\right\|_{0, h}$ or $\left|\boldsymbol{u}_{i}-\boldsymbol{u}^{*}\right|_{1, h}$.

\subsubsection{Slip boundary condition}

In the first $2 \mathrm{D}$ verification setup, the slip boundary condition is evaluated. Our approach in constructing the test problem is to find solutions to the elasticity equation on wedge-shaped domains and then glue two wedge-shaped domains together, separated by a dipping fault.

Let a semi-infinite wedge be given by $\Omega^{\theta_{1}, \theta_{2}}=\{(x, y) \in$ $\left.\mathbb{R}^{2}: \theta_{1}<\theta(x, y)<\theta_{2}\right\}$, where $\theta \in[-\pi, \pi)$ is the polar angle, i.e. $x=r \cos (\theta)$ and $y=r \sin (\theta)$. Then we can construct a dipping fault by gluing domains $\Omega^{A}:=\Omega^{-\pi, \omega}$ and $\Omega^{B}:=\Omega^{\omega, 0}$, where the dip angle $\omega \in(-\pi, 0)$. Here, we set $\omega=-\pi / 3$, as illustrated in Figure $3 \mathrm{a}$. For our test problem we require the following boundary conditions to be satisfied:

$$
\begin{array}{rlr}
\left.\sigma_{r \theta}^{A}\right|_{\theta=-\pi} & =\left.\sigma_{\theta \theta}^{A}\right|_{\theta=-\pi}=0, & \\
\left.\sigma_{r \theta}^{B}\right|_{\theta=0} & =\left.\sigma_{\theta \theta}^{B}\right|_{\theta=0}=0 & \text { (free surface), } \\
\llbracket \sigma_{\theta \theta} \rrbracket & =\left.\sigma_{\theta \theta}^{A}\right|_{\theta=\omega}-\left.\sigma_{\theta \theta}^{B}\right|_{\theta=\omega}=0, & \\
\llbracket \sigma_{r \theta} \rrbracket & =\left.\sigma_{r \theta}^{A}\right|_{\theta=\omega}-\left.\sigma_{r \theta}^{B}\right|_{\theta=\omega}=0, & \text { (continuity), } \\
\llbracket u_{\theta} \rrbracket & =\left.u_{\theta}^{A}\right|_{\theta=\omega}-\left.u_{\theta}^{B}\right|_{\theta=\omega}=0 \quad \text { (no-opening), } \\
\llbracket u_{r} \rrbracket & =\left.u_{r}^{A}\right|_{\theta=\omega}-\left.u_{r}^{B}\right|_{\theta=\omega}=\frac{r^{\gamma}}{2 \mu} & \text { (slip). }
\end{array}
$$

It is convenient to express stress and displacement in terms of a biharmonic function $\phi$ and a harmonic function $\psi$. Following Williams (2021) we then have the plane strain solution

$$
\begin{aligned}
2 \mu u_{r} & =-\frac{\partial \phi}{\partial r}+(1-\nu) r \frac{\partial \psi}{\partial \theta}, \\
2 \mu u_{\theta} & =-\frac{1}{r} \frac{\partial \phi}{\partial \theta}+(1-\nu) r^{2} \frac{\partial \psi}{\partial r}, \\
\sigma_{r r} & =\frac{1}{r} \frac{\partial \phi}{\partial r}+\frac{1}{r^{2}} \frac{\partial^{2} \phi}{\partial \theta^{2}}, \\
\sigma_{\theta \theta} & =\frac{\partial^{2} \phi}{\partial r^{2}}, \\
\sigma_{r \theta} & =-\frac{\partial}{\partial r}\left(\frac{1}{r} \frac{\partial \phi}{\partial \theta}\right),
\end{aligned}
$$

where $\mu$ is the shear modulus, $\nu$ is Poisson's ratio, and $\phi$ and $\psi$ are related by

$\nabla^{2} \phi=\frac{\partial}{\partial r}\left(r \frac{\partial \psi}{\partial \theta}\right)$

We use the ansatz (Williams 2021)

$$
\begin{aligned}
\phi^{A}= & r^{\gamma+1}\left[A_{1} \sin ((\gamma+1) \theta)+A_{2} \cos ((\gamma+1) \theta)\right. \\
& \left.+A_{3} \sin ((\gamma-1) \theta)+A_{4} \cos ((\gamma-1) \theta)\right] \\
\psi^{A}= & r^{\gamma-1}\left[-\frac{4 A_{3}}{\gamma-1} \cos ((\gamma-1) \theta)+\frac{4 A_{4}}{\gamma-1} \sin ((\gamma-1) \theta)\right],
\end{aligned}
$$

on sub-domain $\Omega^{A}$ for constants $\gamma$ and $A_{1}, \ldots, A_{4}$. The ansatz $\phi^{B}, \psi^{B}$ for sub-domain $\Omega^{B}$ is defined likewise but with constants $B_{1}, \ldots, B_{4}$. The constants are determined as following: 

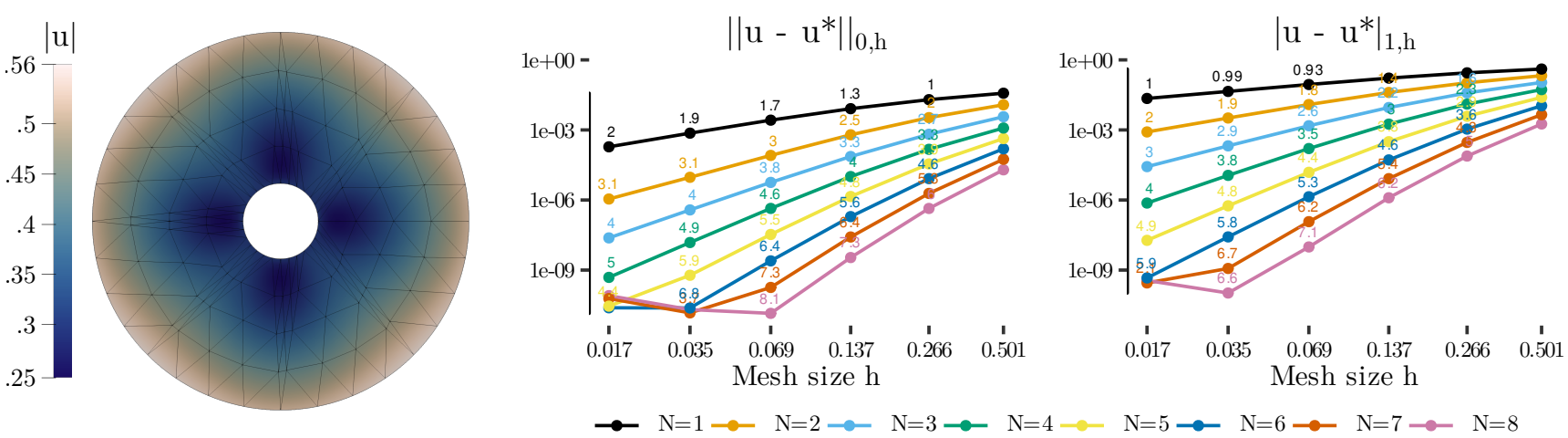

(a) Circular hole verification problem (2D). Left: snapshot of the solution and mesh. Middle and right: Convergence test results.
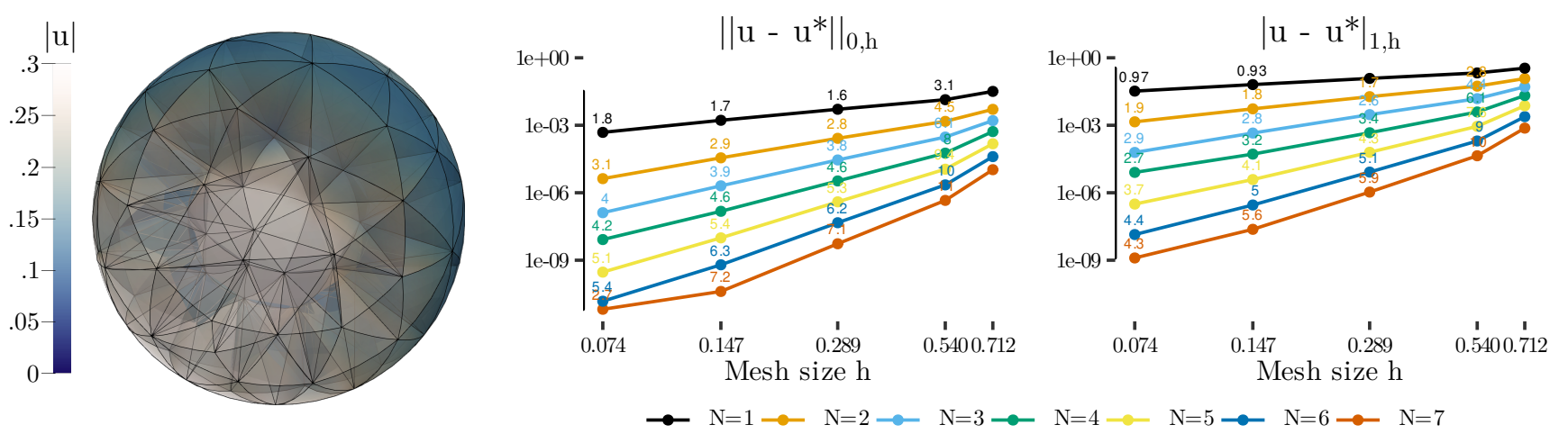

(b) Spherical hole verification problem (3D). Left: snapshot of the solution and mesh. Middle and right: Convergence test results.

Figure 4. Results of the circular and spherical hole convergence tests which verify the curvilinear grids. The empirical convergence order, printed in small text above points, mostly matches the theoretical expectations.

(i) Use the free surface conditions to express $A_{1}, A_{2}, B_{1}, B_{2}$ in terms of $A_{3}, A_{4}, B_{3}, B_{3}$.

(ii) Add the slip equation and the continuity equation for $\sigma_{\theta \theta}$. Subtract the no-opening equation from the continuity equation for $\sigma_{r \theta}$. Use the resulting two equations to express $A_{3}$ in terms of $B_{3}$ and $A_{4}$ in terms of of $B_{4}$.

(iii) Find the remaining constants $B_{3}$ and $B_{4}$ from the continuity equations.

In practice, we use SymPy (Meurer et al. 2017) to execute the above steps. The full procedure is provided in an openly available Jupyter notebook (see DATA AVAILABILITY). Furthermore, to avoid having to deal with infinite domains, we limit the A and B domains to $r \leq 1$. At the "new" boundary at $r=1$ we impose the solution as Dirichlet boundary condition.

At last, we discuss the choice of the smoothness parameter $\gamma$. The term $\sin (\pi \gamma)$ appears in the denominator of $B_{3}$ and $B_{4}$, thus we must choose $\gamma \in \mathbb{R} \backslash \mathbb{Z}$. Moreover, the term $r^{\gamma-1}$ appears in the stress tensor. Hence, $\gamma>0$ in order to bound the strain energy (Barber 2004, §11.2.1). More generally, the $k$-th derivative of the displacement contains the term $r^{\gamma-k}$. The norm of this term on a wedge-shaped domain with maximum radius $r_{\max }$, i.e. $\left(\int_{\theta_{1}}^{\theta_{2}} \int_{0}^{r_{\max }} r^{2(\gamma-k)} r \mathrm{~d} r \mathrm{~d} \theta\right)^{1 / 2}$, is only bounded if $\gamma>k-1$. Consequently, $\gamma$ controls the smoothness of the solution. E.g. for $0<\gamma<1$ the solution cannot be element of $H^{k}$ with $k \geq 2$ and hence one cannot achieve high-order convergence using the SIPG method (Rivière 2008).

Here, we analyse the cases $\gamma=0.5$ and $\gamma=10.5$. The constants of the respective solution are listed in Table 2.
Table 2. Numerical parameters for the dipping fault static test case.

\begin{tabular}{rrr}
\hline & $\gamma=0.5$ & $\gamma=10.5$ \\
\hline$A_{1}$ & 0.20833333333333 & -0.16666666666667 \\
$A_{2}$ & 0.072168783648703 & 2.7424137786507 \\
$A_{3}$ & -0.20833333333333 & 0.16666666666667 \\
$A_{4}$ & 0.21650635094611 & -3.3197640478403 \\
$B_{1}$ & -0.125 & $-3.3017067341026 \cdot 10^{-15}$ \\
$B_{2}$ & 0.072168783648703 & 3.0310889132455 \\
$B_{3}$ & -0.375 & $3.9968028886506 \cdot 10^{-15}$ \\
$B_{4}$ & -0.072168783648703 & -3.0310889132455 \\
\hline
\end{tabular}

\subsubsection{Circular hole}

In the second $2 \mathrm{D}$ verification problem, we evaluate whether highorder convergence is achieved for a curved boundary. To this end, we let the domain be the unit disk with a small hole, that is, for hole-radius $a$ we have $\Omega^{a}=\left\{\boldsymbol{x} \in \mathbb{R}^{2}: a<|\boldsymbol{x}|<1\right\}$. The domain is illustrated in Figure 4a for $a=0.2$. The hole's boundary is required to be traction free, i.e.

$\sigma_{r r}=\sigma_{r \theta}=0, \quad \forall \boldsymbol{x} \in \mathbb{R}^{2}:|\boldsymbol{x}|=a$.

The solution ansatz is the biharmonic function

$\phi=-\frac{S_{0}}{2} r^{2} \sin (2 \theta)+A \sin (2 \theta)+B r^{-2} \sin (2 \theta)$,

for constants $S_{0}, A, B$ (Barber 2004, §8.3.2). From the traction free boundary conditions it follows that

$A=S_{0} a^{2}, \quad B=-\frac{S_{0} a^{4}}{2}$. 

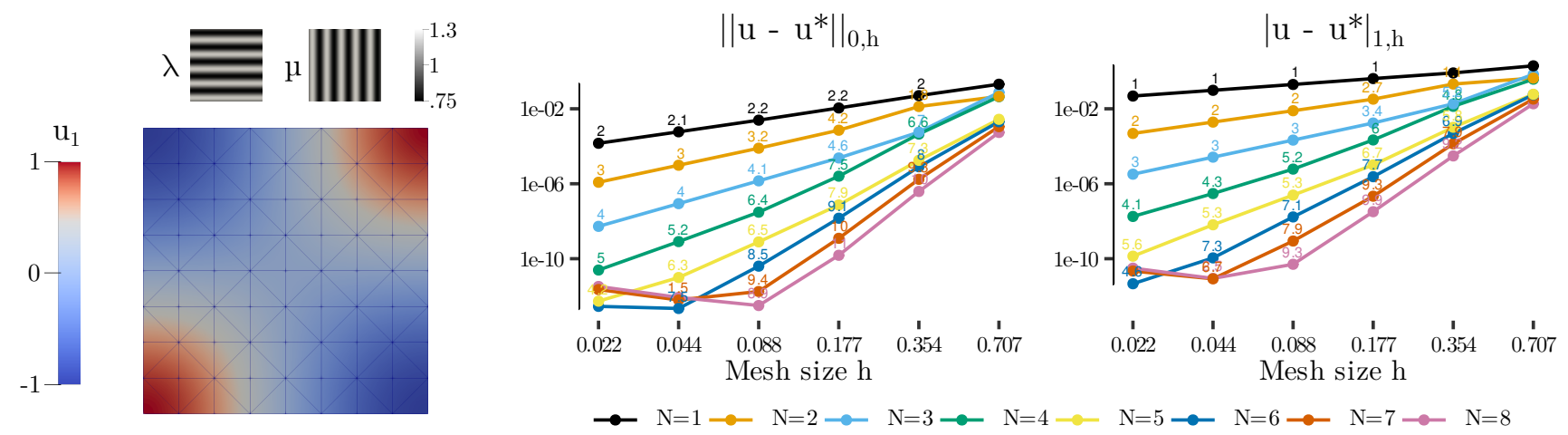

Figure 5. Verification of heterogeneous material parameters. The empirical convergence order, printed in small text above points, mostly matches the theoretical expectations.

A harmonic function satisfying the compatibility condition (63) is

$\psi=-2 A r^{-2} \cos (2 \theta)$.

Hence, a displacement field satisfying the elasticity equations and (65) is given by

$2 \mu u_{r}=S_{0} r^{-3}\left(r^{4}+4 a^{2} r^{2}(1-\nu)-a^{4}\right) \sin (2 \theta)$,

$2 \mu u_{\theta}=S_{0} r^{-3}\left(r^{4}+2 a^{2} r^{2}(1-2 \nu)+a^{4}\right) \cos (2 \theta)$.

We set $S_{0}=1$ and impose (69) on the outer circular boundary $(r=1)$ as Dirichlet boundary condition.

\subsubsection{Spherical hole}

The spherical hole verification problem is the three-dimensional analogue of the circular hole test case. The domain is given by $\Omega^{a}=\left\{\boldsymbol{x} \in \mathbb{R}^{3}: a<|\boldsymbol{x}|<1\right.$, cf. Figure $4 \mathrm{~b}$ for hole radius $a=0.5$. In spherical coordinates, that is,

$x=R \cos (\theta) \sin (\beta), y=R \sin (\theta) \sin (\beta), z=R \cos (\beta)$,

where $\theta \in[-\pi, \pi)$ and $\beta \in[0, \pi)$, we look for a displacement field which satisfies

$\sigma_{R R}=\sigma_{R \theta}=\sigma_{R \beta}=0, \quad \forall \boldsymbol{x} \in \mathbb{R}^{3}:|\boldsymbol{x}|=a$.

Barber (2004, §24.1.2) gives potentials $\phi, \omega$ from which stress fields satisfying (71) are derived. The displacement field can be computed from these potentials (Barber 2004, Table 19.2). After simplifying the resulting expressions, we have $u_{\theta}=0$ and

$$
\begin{aligned}
2 \mu u_{R} & =S_{0}\left(\frac{R(1-\nu)}{2(1+\nu)}+\frac{a^{3}(10 \nu-13)}{4 R^{2}(5 \nu-7)}+\frac{3 a^{5}}{4 R^{4}(5 \nu-7)}\right) \\
& +S_{0}\left(\frac{R}{2}+\frac{5 a^{3}(4 \nu-5)}{4 R^{2}(5 \nu-7)}+\frac{9 a^{5}}{4 R^{4}(5 \nu-7)}\right) \cos (2 \beta), \\
2 \mu u_{\beta} & =-\frac{R S_{0}}{2}\left(\frac{5 R^{2} a^{3}(1-2 \nu)+3 a^{5}}{R^{5}(7-5 \nu)}+1\right) \sin (2 \beta) .
\end{aligned}
$$

We set $S_{0}=1$ and impose (72) on the outer sphere $(r=1)$ as a Dirichlet boundary condition.

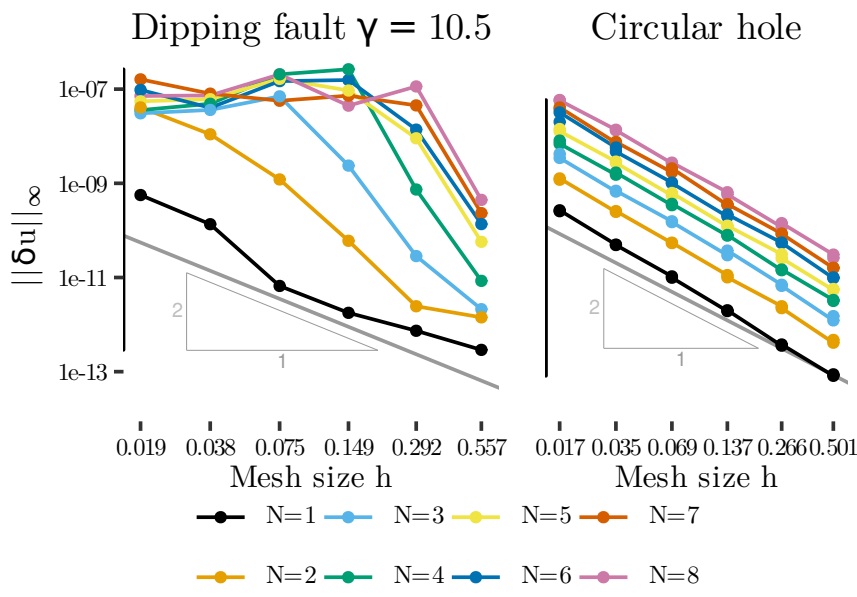

Figure 6. The conditioning of linear systems amplifies errors in the residual, that is, the error $\underline{\boldsymbol{\delta} \boldsymbol{u}}$ might be large even if the residual is reduced down to machine precision. Here, we show bounds on the error $\underline{\boldsymbol{\delta} \boldsymbol{u}}$ obtained by MUMPS for two scenarios. The $h^{-2}$ scaling is added for reference in gray.

\subsubsection{Heterogeneous material parameters}

The material parameters are heterogeneous and set to

$\mu(\boldsymbol{x})=1+\sin \left(10 \pi x_{1}\right) / 4$,

$\lambda(\boldsymbol{x})=1+\sin \left(10 \pi x_{2}\right) / 4$.

The solution is manufactured to be

$\boldsymbol{u}^{*}(\boldsymbol{x})=\left(\cos \left(\pi x_{1}\right) \cos \left(\pi x_{2}\right), \quad 0\right)$.

We ensure that the manufactured solution is a solution of (12) by using only Dirichlet boundaries with $\boldsymbol{g}^{D}=\boldsymbol{u}^{*}$ and

$f_{i}=-\frac{\partial \sigma_{i j}\left(\boldsymbol{u}^{*}\right)}{\partial x_{j}}$.

The force term is computed using the symbolic math package SymPy (Meurer et al. 2017). The domain is chosen as the unit square as illustrated in Figure 5.

\subsubsection{Results}

We conduct a convergence study for the four described test problems for polynomial degrees $N=1, \ldots, 8$. Again, mesh sequences are generated with Gmsh version 4.8.4 (Geuzaine \& 
Table 3. Circular hole verification problem on an affine mesh sequence. Shown are the errors and empirical convergence orders on the finest mesh ( $h=0.017$ ). The convergence order is limited due to linear approximation of the inner and outer circle.

\begin{tabular}{rrrrr}
\hline$N$ & $\left|u-u^{*}\right|_{1, h}$ & $\mathcal{C}_{1, h}$ & $\left\|u-u^{*}\right\|_{0, h}$ & $\mathcal{C}_{0, h}$ \\
\hline 1 & $2.23271 \mathrm{e}-02$ & 1.0 & $1.89689 \mathrm{e}-04$ & 2.0 \\
2 & $8.23588 \mathrm{e}-04$ & 2.0 & $3.02087 \mathrm{e}-06$ & 2.4 \\
3 & $5.53858 \mathrm{e}-05$ & 2.2 & $2.62902 \mathrm{e}-06$ & 2.0 \\
4 & $7.07560 \mathrm{e}-05$ & 1.6 & $2.62783 \mathrm{e}-06$ & 2.0 \\
5 & $9.18152 \mathrm{e}-05$ & 1.5 & $2.62678 \mathrm{e}-06$ & 2.0 \\
6 & $1.16266 \mathrm{e}-04$ & 1.5 & $2.62560 \mathrm{e}-06$ & 2.0 \\
7 & $1.43651 \mathrm{e}-04$ & 1.5 & $2.62449 \mathrm{e}-06$ & 2.0 \\
8 & $1.69295 \mathrm{e}-04$ & 1.5 & $2.62369 \mathrm{e}-06$ & 2.0 \\
\hline
\end{tabular}

Remacle 2009; Johnen et al. 2013). Based on a CAD model, an initial degree 8 coarse mesh is generated and successively refined such that the mesh size is roughly halved w.r.t. the previous mesh in the sequence. The heterogeneous material parameter verification setup is an exception, here we use an uniform mesh generator for rectangular domains. If the solution is smooth enough, the convergence order is expected to be $N+1$ for displacement and $N$ for the first derivatives (Castillo et al. 2000; Rivière 2008)

We first discuss the numerical convergence results for the slip boundary condition. For the case $\gamma=0.5$ we showed in Sec. 8.1.1 that only the first (weak) derivative of the displacement solution exists. Thus, a convergence order below one is expected independent of the polynomial degree $N$ (Rivière 2008). Empirically, we find that the convergence order is about 0.5 , cf. Figure $3 \mathrm{~b}$. Still, the error is reduced by a constant factor with increasing polynomial degree, for example the error for $N=8$ is one tenth of the error for $N=1$ and $N=8$ on the finest mesh. For the case $\gamma=10.5$ the empirical convergence order is close the expected order $N$ in the $|\cdot|_{1, h}$ semi-norm, but we observe that the error is not reduced below $5 \cdot 10^{-10}$. The condition number of the operator $\boldsymbol{A}$ is in $\mathcal{O}\left(h^{-2}\right)$ (Antonietti \& Houston 2011), hence even when reducing the residual down to machine precision the error might be much larger than machine precision.

A posteriori error analysis of the linear solve is offered by the direct sparse solver MUMPS (Amestoy et al. 2001, 2019) using the method of Arioli et al. (1989). The error analysis returns a bound on $\underline{\boldsymbol{\delta} \boldsymbol{u}}$, that is, a bound on the error introduced in the degrees of freedom by finite precision effects. The results of the error analysis for the dipping fault test case and the circular hole test case are shown in Figure 6. We see that an error in the degrees of freedom of at most $2 \cdot 10^{-7}$ in the infinity norm is possible. Therefore, the minimum achievable error in Figure $3 \mathrm{~b}$ is explained by the amplification of the error in the residual due to conditioning of the linear system. Moreover, the error bound roughly follows the $h^{-2}$ scaling of the condition number.

We next discuss the numerical convergence results of the circular hole and spherical hole verification cases shown in Figure 4. For degrees $N=1, \ldots, 6$ the empirical convergence orders match the theoretical expectation very well in both the $\|\cdot\|_{0, h}$ norm and $|\cdot|_{1, h}$ semi-norm. For degrees $N=7,8$ the empirical orders are slightly off but still increase with increasing $N$. We also repeated the test for an affine mesh (i.e. degree 1), as shown in Table 3. Due to the linear approximation of the boundary the convergence order in the $\|\cdot\|_{0, h}$ norm is reduced to 2 (with a curious exception for $N=2$ where it is slightly higher), which shows that the curvilinear geometry approximation is necessary to retain high-order convergence. In the spherical hole test case theoretical expectations are well met until $N=3$. The empirical convergence orders for higher degrees are lower than expected but still increase with increasing $N$. The condition number of the circular hole test case increases with $h^{-2}$, as shown in Figure 6, such that the error is not reduced below $10^{-11}$, and, once the minimum attainable error is reached, gets slightly worse under mesh refinement.

Lastly, high-order convergence is also achieved when material parameters are smoothly varying in space, cf. Figure 5.

\subsection{Convergence of the SEAS solver}

Establishing convergence of a SEAS model is difficult as there are no known analytic solutions. However, by adding a source term to the state equation one can manufacture a solution. Erickson \& Dunham (2014) manufactured such a solution for a two-dimensional anti-plane shear problem (where the elasticity equations reduce to a Poisson equation). Here, we propose a manufactured verification problem for plane strain elasticity.

\subsubsection{Domain}

Assume that the fault lies in the $x_{1}=0$ plane. The first step is to construct a displacement field possessing a tangential component which is discontinuous at the fault, but with a normal component and traction which are continuous. That is, $u_{1}, \sigma_{11}$, and $\sigma_{12}$ are continuous but $u_{2}$ is discontinuous. We find that the Airy stress function (Barber 2004)

$\Phi^{ \pm}(\boldsymbol{x})=x_{1} K(t) \exp \left(-k^{ \pm} x_{1}\right) \cos \left(k^{ \pm} x_{2}\right)$

leads to a displacement field with the desired properties. $K(t)$ is a time-dependent function and we define $k^{+}=k$ and $k^{-}=-k$ for a constant $k$. Indeed, the stress and displacement components are

$\sigma_{11}^{ \pm}=-k^{2} x_{1} K \exp \left(-k^{ \pm} x_{1}\right) \cos \left(k^{ \pm} x_{2}\right)$

$\sigma_{22}^{ \pm}=k^{ \pm}\left(k^{ \pm} x_{1}-2\right) K \exp \left(-k^{ \pm} x_{1}\right) \cos \left(k^{ \pm} x_{2}\right)$,

$\sigma_{12}^{ \pm}=k^{ \pm}\left(1-k^{ \pm} x_{1}\right) K \exp \left(-k^{ \pm} x_{1}\right) \sin \left(k^{ \pm} x_{2}\right)$,

$u_{1}^{ \pm}=\frac{k^{ \pm} x_{1}(\nu+1)-2 \nu^{2}-\nu+1}{E} K \exp \left(-k^{ \pm} x_{1}\right) \cos \left(k^{ \pm} x_{2}\right)$,

$u_{2}^{ \pm}=\frac{k^{ \pm} x_{1}(\nu+1)+2 \nu^{2}-2}{E} K \exp \left(-k^{ \pm} x_{1}\right) \sin \left(k^{ \pm} x_{2}\right)$,

where $E$ is Young's modulus and $\nu$ is Poisson's ratio. For $x_{1}=0$ we have $\sigma_{11}^{-}=\sigma_{11}^{+}, \sigma_{12}^{-}=\sigma_{12}^{+}, u_{1}^{-}=u_{1}^{+}$, but

$\llbracket u_{2} \rrbracket=u_{2}^{-}-u_{2}^{+}=4 \frac{1-\nu^{2}}{E} K \sin \left(k x_{2}\right)$.

Following Erickson \& Dunham (2014), we choose $K$ as

$K(t)=\frac{2}{\pi}\left(\arctan \left(\frac{t-t_{e}}{t_{w}}\right)+\frac{\pi}{2}\right)$,

such that there will be one "event" at time $t_{e}$ with a duration of about $t_{w}$ (cf. Table 4).

The domains are $\Omega^{-}=(-3 / 5,0) \times(-4 / 5,-1 / 5)$ and $\Omega^{+}=(0,3 / 5) \times(-4 / 5,-1 / 5)$. On the internal fault boundary $\Gamma^{F}=\{0\} \times[-4 / 5,-1 / 5]$ we impose the slip $\llbracket u_{2} \rrbracket$. On the remaining boundary, $\Gamma^{D, \pm}=\partial \Omega^{ \pm} \backslash \Gamma^{F}$ we impose the displacement field from (77). 
Table 4. Parameters for the 2D plane strain SEAS model.

\begin{tabular}{ll|ll}
\hline Parameter & Value & Parameter & Value \\
\hline$t_{e}$ & $50 \mathrm{~s}$ & $a$ & 0.015 \\
$t_{w}$ & $1 \mathrm{~s}$ & $b$ & 0.02 \\
$\rho_{0}$ & $2670 \mathrm{~kg} / \mathrm{m}^{3}$ & $V_{0}$ & $1 \times 10^{-6} \mathrm{~m} \mathrm{~s}^{-1}$ \\
$c_{s}$ & $3.464 \mathrm{~km} \mathrm{~s}^{-1}$ & $f_{0}$ & 0.6 \\
$\nu$ & 0.25 & $k$ & $\pi$ \\
$E$ & $2 \mathrm{c}_{\mathrm{s}}^{2} \rho_{0}(1+\nu) \mathrm{Pa}$ & $\sigma_{n}^{0}$ & $25\left|\sin \left(\mathrm{kx}_{2}\right)\right| \mathrm{MPa}$ \\
$\eta$ & $\rho_{0} \mathrm{c}_{\mathrm{s}} / 2 \mathrm{Pas} \mathrm{m} \mathrm{m}^{-1}$ & $T^{0}$ & $20 \sin \left(\mathrm{kx}_{2}\right) \mathrm{MPa}$ \\
\hline
\end{tabular}

\subsubsection{Fault}

We add a source term independent of state $\psi$ and slip $S$ to the state equation, that is,

$\frac{\mathrm{d} \psi}{\mathrm{d} t}=G(V, \psi)+s(\boldsymbol{x}, t)$.

The source term is defined as

$s(\boldsymbol{x}, t)=-G\left(V^{*}, \psi^{*}\right)+\frac{\mathrm{d} \psi^{*}}{\mathrm{~d} t}$,

where $V^{*}(\boldsymbol{x}, t)$ and $\psi^{*}(\boldsymbol{x}, t)$ are the manufactured solutions. Given that $V(\boldsymbol{x}, 0)=V^{*}(\boldsymbol{x}, 0)$ and $\psi(\boldsymbol{x}, 0)=\psi^{*}(\boldsymbol{x}, 0)$ the fields $V^{*}, \psi^{*}$ are a solution for (80).

The manufactured slip $S^{*}$ needs to be compatible with the solution in the domain, in particular with (78). With the fault normal $(1,0)$ and tangent $(0,-1)$ we have $S^{*}:=-\llbracket u_{2} \rrbracket$. It follows that the manufactured slip-rate is given by

$V^{*}=-4 \frac{1-\nu^{2}}{E} \frac{\mathrm{d} K}{\mathrm{~d} t} \sin \left(k x_{2}\right)$.

Likewise, from (77) the manufactured on-fault normal stress and shear stress are

$\sigma_{n}^{*}=\sigma_{n}^{0}-\left.\sigma_{11}\right|_{x_{1}=0}=\sigma_{n}^{0}$,

$T^{*}=T^{0}-\left.\sigma_{12}\right|_{x_{1}=0}=T^{0}-k K \sin \left(k x_{2}\right)$,

with background stress $\sigma_{n}^{0}, T^{0}$.

Lastly, the manufactured quantities need to satisfy the friction law. From (6) and (10) we have

$-T^{*}=\sigma_{n}^{*} a \operatorname{arcsinh}\left(\frac{V^{*}}{2 V_{0}} \exp \left(\frac{\psi^{*}}{a}\right)\right)+\eta V^{*}$.

Solving the above equation for $\psi^{*}$ we obtain

$\psi^{*}=a \ln \left(\frac{2 V_{0}}{V^{*}} \sinh \left(-\frac{T^{*}+\eta V^{*}}{\sigma_{n}^{*} a}\right)\right)$.

The parameters $a, V_{0}, \eta$ as well the background stress and all other parameters are summarised in Table 4.

\subsubsection{Results}

We conduct a convergence study for polynomial degrees $N=$ $1, \ldots, 8$. The mesh sequence is generated with an uniform mesh generator for rectangular domains. The final simulation time is set to $t_{\text {end }}=2 t_{e}=100 \mathrm{~s}$. We report the error at $t_{\mathrm{end}}$, where the timedependent error is given by

$$
\left(\sum_{e \in \Gamma^{F}} \int_{e}\left(\psi-\psi^{*}\right)^{2}+\left(S-S^{*}\right)^{2} \mathrm{~d} s\right)^{1 / 2} .
$$

Time-stepping is handled by PETSc/TS (Abhyankar et al.

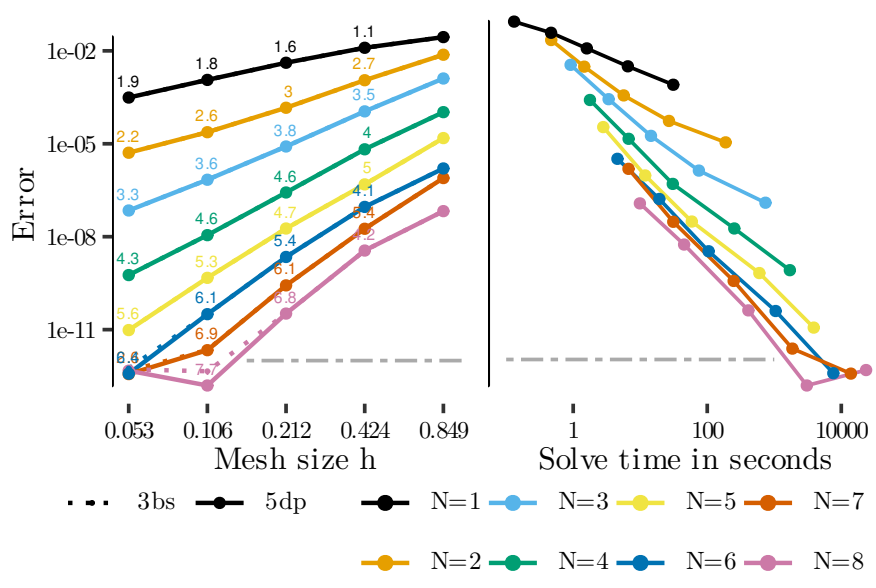

Figure 7. Results of convergence analysis for the manufactured SEAS problem. Left: The empirical convergence order, printed in small text above points, is close to the polynomial degree $N$ in almost all cases. Right: The error vs. time-to-solution plot shows that high-order pays off for this problem. The absolute tolerance of $10^{-12}$ used to select time-steps is indicated by the gray dashed line. The error reported on the y-axis is given by (86).

2018; Balay et al. 2021a). We use adaptive Runge-Kutta methods such as the third order Bogacki-Shampine scheme (3bs) with the second order embedded method or the fifth order DormandPrince scheme (5dp) with the fourth order embedded method. The local truncation error is estimated by the difference between the fifth (third) order method and the fourth (second) order embedded method. Time-step sizes are adapted automatically such that the estimated local truncation error stays below a given absolute tolerance. We set an absolute tolerance of $10^{-12}$ and find that the choice of time-stepping method has little impact when the error is above the error tolerance, cf. the solid and dotted lines in Figure 7. Therefore, we only discuss the results of method 5dp.

The effective frictional resistance is related to traction via the empirical rate and state friction law, thus, we expect a maximum convergence order of $N$. The results in Figure 7 show that order $N$ is reached, although the absolute error tolerance limits the minimum achievable error. Curiously, for $N=1$ the empirical convergence order is close to two.

Plotting the error versus the solve time (Figure 7) shows that high-order clearly pays off. E.g. for $N=1$ the error on the finest grid is $3.1 \times 10^{-4}$ and the solve time increases by about a factor of 4 when the mesh size is halved. Assuming second order con-

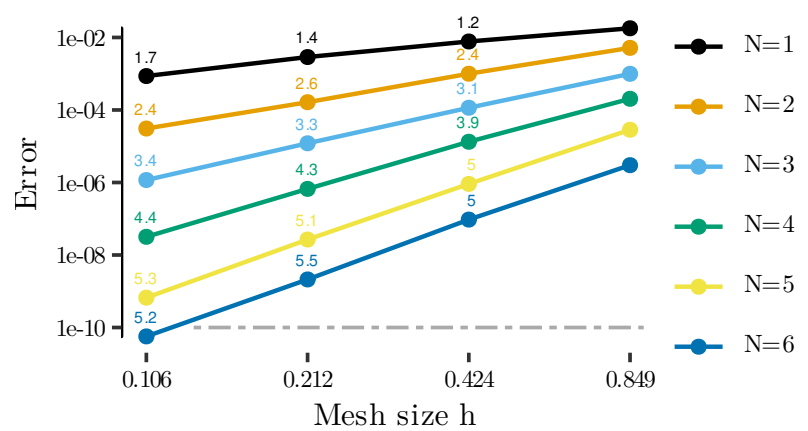

Figure 8. Convergence results for the manufactured 3D SEAS problem. The absolute tolerance of $10^{-10}$ used to select time-steps is indicated by the gray dashed line. The error reported on the y-axis is given by (86). 
vergence, one needs about 8 refinement steps to reach an error of $1 \times 10^{-8}$ Therefore, an increase in solve time by $4^{8}$ is necessary, that is, about 24 days. In contrast, a solve time of about $45 \mathrm{~s}$ is required to fall below the same error threshold for the $N=8$ scheme.

We extend the manufactured verification setup in a straightforward manner to three dimensions, essentially by solving the $2 \mathrm{D}$ problem with the $3 \mathrm{D}$ scheme. We run the $3 \mathrm{D}$ test case for polynomial degrees $N=1, \ldots, 6$ setting an absolute tolerance of $1 \times 10^{-10}$. The results are shown in Figure 8. The empirical convergence order is close to $N$.

\subsection{SEAS Benchmark Problem 1 (BP1)}

Another means of verification is a code comparison in which a representative physical problem is defined and solved by different groups, with different numerical methods and implementations (Harris et al. 2018; Buiter et al. 2016, e.g.,), and the results of all approaches are subsequently compared. While this approach does not reveal the "ground truth", we can analyse the variation between codes. A community code comparison is organised by the SEAS working group (Erickson et al. 2020) of the Southern California Earthquake Center (SCEC). We use their Benchmark Problem 1 (BP1) to test several aspects of our method: First, we investigate whether local (statically adaptive) mesh refinement is effective in comparison to uniform mesh refinement. Second, we check whether p-refinement improves our results significantly for the BP1 problem where the regularity of the solution is unknown. Lastly, we verify that a high-resolution simulation with our method matches the results of other groups.

\subsubsection{Local h-refinement}

One of the issues raised by Erickson et al. (2020) when using volume based discretisations for BP1 is the unknown effect of the prescribed finite sized domain on the solution. Using an unstructured mesh, addressing this issue can be tackled efficiently. In the following, we chose a $2 \mathrm{D}$ volumetric domain as a $[0 \mathrm{~km}, 400 \mathrm{~km}] \times$ $[-400 \mathrm{~km}, 0 \mathrm{~km}]$ rectangle. Four unstructured meshes were constructed with approximately the same number of elements, but with differing spatial resolution on-fault $\left(h_{f}\right)$ and off-fault. The off-fault resolution is controlled by specifying the spatial resolution $\left(h_{b}\right)$ on the exterior boundary not containing the fault. In practice we selected $h_{f}$ and adjusted $h_{b}$ to produce a mesh with approximately 15000 cells. The four meshes (shown in Figure 9) were defined using $h_{f}=\{5000,1000,100,25\} \mathrm{m}$. The end-members $\left(\mathcal{T}_{h}^{A}, \mathcal{T}_{h}^{D}\right)$ have an approximately uniform spatial resolution of $5 \mathrm{~km}$, c.f. an on-fault resolution of $25 \mathrm{~m}$ and an off-fault resolution of $130 \mathrm{~km}$.

In Figure 10 we report the simulated shear stress over five seismic cycles using the four different meshes and a degree $N=2$ polynomial. For cases when $h_{f} \geq 1 \mathrm{~km}$, our solutions dramatically deviate from the reference solution (grey line in Figure 10). In comparison, the higher resolution simulations using an on-fault resolution of $100 \mathrm{~m}$ (green line $-\mathcal{T}_{h}^{C}$ ) and $25 \mathrm{~m}$ (black line $-\mathcal{T}_{h}^{D}$ ) are seen to closely follow the reference in terms of both, phase and amplitude.

\subsection{2 p-refinement}

To highlight the benefits of using high polynomial degree function spaces, we conducted simulations using $\mathcal{T}_{h}^{B}$ and vary the polynomial degree $N$. The rational for selecting one of the "low res-
Table 5. Recurrence time $\left(\Delta t_{r}\right)$ estimates from last cycle associated with the simulations reported in Figure 10 and Figure 11. The simulation marked with '-' indicate that a stable periodic sequence of events was not obtained. The reference $\Delta t_{r}$ from C. Cattania was $78.34 \mathrm{yr}$.

\begin{tabular}{crrr}
\hline Mesh & $h_{f}(\mathrm{~m})$ & $N$ & $\Delta t_{r}(\mathrm{yr})$ \\
\hline $\mathcal{T}_{h}^{A}$ & 5000 & 2 & - \\
$\mathcal{T}_{h}^{B}$ & 1000 & 2 & 108.6 \\
$\mathcal{T}_{h}^{C}$ & 100 & 2 & 78.22 \\
$\mathcal{T}_{h}^{D}$ & 25 & 2 & 78.28 \\
\hline $\mathcal{T}_{h}^{B}$ & 1000 & 4 & 89.5 \\
$\mathcal{T}_{h}^{B}$ & 1000 & 6 & 82.9 \\
$\mathcal{T}_{h}^{B}$ & 1000 & 8 & 77.6 \\
\hline
\end{tabular}

olution" meshes (as inferred from the cell sizes $h_{f}, h_{b}$ ) is that with higher $N$, we expect that higher spatial resolution should be achieved even if the cell sizes $h$, where $h_{f} \leq h \leq h_{b}$, are in fact large. In Figure 11 this expectation is seen to be correct. For low degrees $(N=2,4)$ the solutions obtained are inaccurate in terms of phase, amplitude and number of cycles. The degree $N=6$ results (purple line) are very similar to the reference solution (grey line) with a slight phase shift. The highest degree used $N=8$ is visually seen to yield the closest agreement with the reference solution. Despite the small phase shift associated with $N=8$ simulations, the actual recurrence interval is very similar to the reference, and more accurate than that obtained from the lower degree experiments (see Table 5).

\subsubsection{Code comparison}

We compare a high-resolution run using our method $(N=8)$ with other codes. The domain is chosen as the $[0 \mathrm{~km}, 400 \mathrm{~km}] \times$ $[-400 \mathrm{~km}, 0 \mathrm{~km}]$ rectangle. Note that only half of the domain needs to be modelled due to symmetry. The boundaries $y=$ $-400 \mathrm{~km}$ and $y=0 \mathrm{~km}$ are traction-free and we impose the displacement $V_{p} t / 2, V_{p}=1 \times 10^{-9} \mathrm{~m} \mathrm{~s}^{-1}$, on the $x=400 \mathrm{~km}$ boundary. The on-fault mesh size is $250 \mathrm{~m}$ and the mesh size is gradually coarsened to $50 \mathrm{~km}$ towards the far boundaries for a total of 3648 triangles. We note that the on-fault mesh size is 10 times larger than the $25 \mathrm{~m}$ mesh size recommended for BP1.

The slip-rate at $7.5 \mathrm{~km}$ depth is compared to eight different reference solutions taken from Erickson et al. (2020) in Figure 12. Overall, we find good agreement in the long-term behaviour. The largest deviation is seen in the onset time of the later earthquake events, which might result from different choices of domain size, as discussed in Erickson et al. (2020). Yet, overlaying the eighth event and correcting for the time shift (Figure 12, lower panel) shows that the coseismic behaviour closely matches the other codes.

\section{NUMERICAL EXPERIMENTS}

We apply the SIPG method to two geometrically complex demonstration models and elucidate the importance of using curvilinear fault representations, compared with piece-wise linear approximations (affine), when accurate stresses (or displacement gradients) are required on-fault. The first demonstrator consists of a 2D SEAS model defined by a shallowly dipping normal fault interacting with four curved splay faults. In the second demonstrator, we consider a 3D elasto-static model defining a kinematic scenario of instantaneous deformation inspired by the 2019 Ridgecrest earthquake se- 


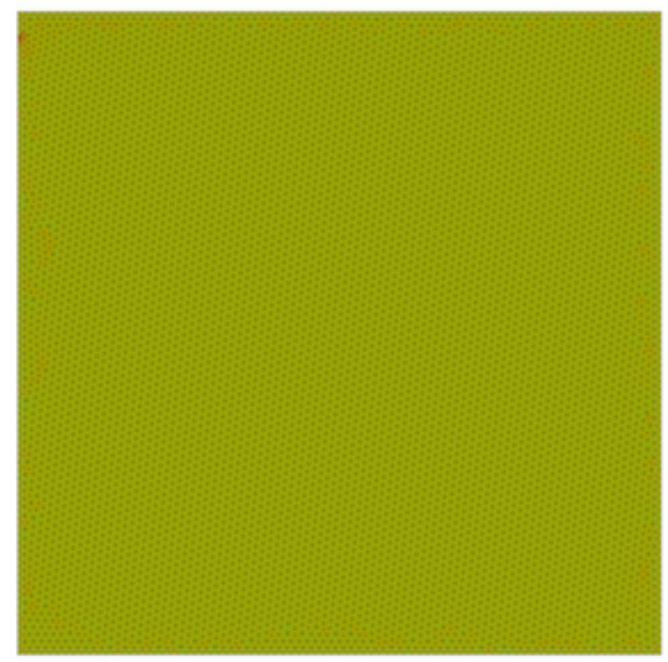

(a) $\mathcal{T}_{h}^{A}: h_{f}=5 \mathrm{~km}, h_{b}=5 \mathrm{~km}, N_{c}=14982$

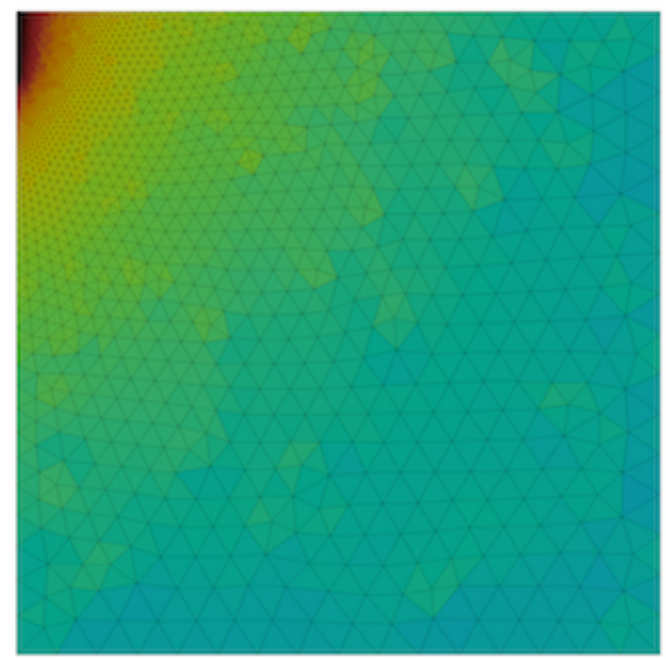

(c) $\mathcal{T}_{h}^{C}: h_{f}=100 \mathrm{~m}, h_{b}=25 \mathrm{~km}, N_{e}=15289$

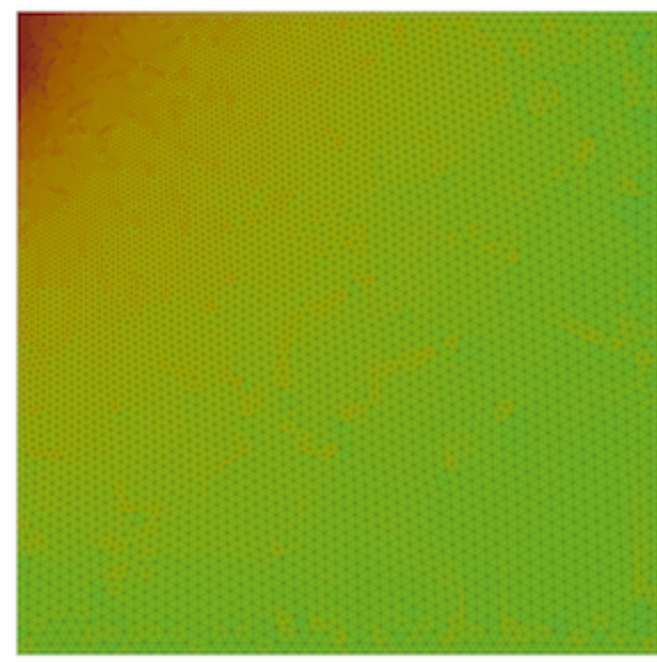

\section{Cell area}

(b) $\mathcal{T}_{h}^{B}: h_{f}=1 \mathrm{~km}, h_{b}=8.5 \mathrm{~km}, N_{e}=15296$

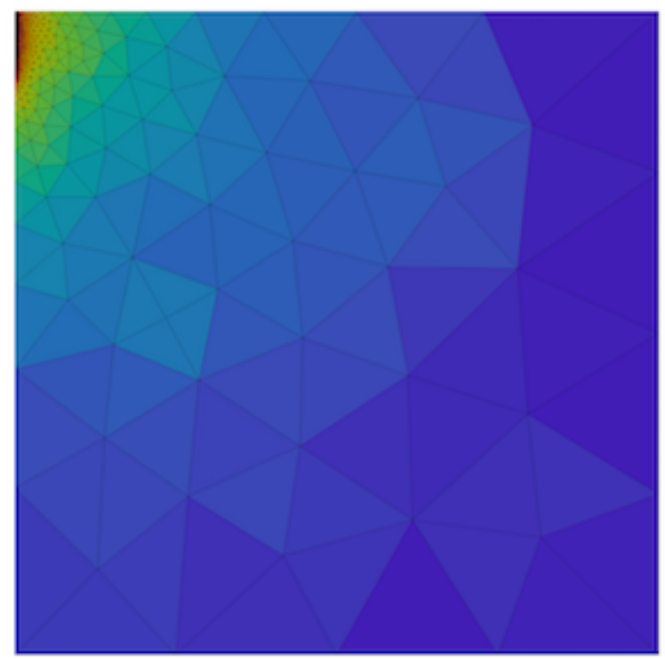

(d) $\mathcal{T}_{h}^{D}: h_{f}=25 \mathrm{~m}, h_{b}=130 \mathrm{~km}, N_{e}=14314$

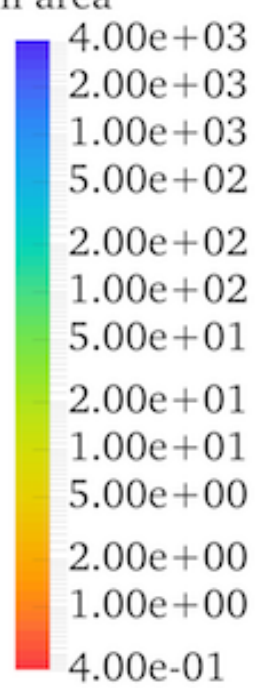

4.00e-01

Figure 9. Family of unstructured meshes $\left(\mathcal{T}_{h}^{\{A, B, C, D\}}\right.$ ) used for BP1, each with approximately the same number of elements $N_{e}$. Each mesh is constructed by specifying a target spatial resolution (triangle edge length) along the fault $\left(h_{f}\right)$ and elsewhere along the exterior boundary $\left(h_{b}\right)$. The degree of local refinement is emphasised by colouring cells according to their area $\left(\mathrm{km}^{2}\right)$ where red hues indicates regions of high local spatial resolution and blue hues denote regions of low local spatial resolution.

quence. Both applications are here presented as proof-of-concepts. Detailed geophysical analysis and including further realistic modeling ingredients, such as subsurface material heterogeneity, will be included in future work.

\subsection{A 2D SEAS multi-fault scenario on a shallowly dipping normal fault with four curved splay faults}

SEAS simulations including more than one fault segment and complex fault geometries are methodologically challenging (Romanet et al. 2018; Galvez et al. 2020; Luo et al. 2020; Barbot 2021) (see also Sec. 10.2), but crucial to understand large earthquakes that often span several segments of natural regional-scale fault networks (Plesch et al. 2007; Ando \& Kaneko 2018; Ulrich et al. 2019; Harris et al. 2021). The mechanics of splay fault systems are of specific interest to seismic and tsunami hazard assessment and to understand
Table 6. Parameters for the splay fault scenario.

\begin{tabular}{ll|ll}
\hline Parameter & Value & Parameter & Value \\
\hline$a_{\max }$ & 0.025 & $a_{0}$ & 0.010 \\
$L$ & $0.05 \mathrm{~m}$ & $b$ & 0.015 \\
$f_{0}$ & 0.6 & $\nu$ & 0.25 \\
$\rho_{0}$ & $2670 \mathrm{~kg} / \mathrm{m}^{3}$ & $c_{s}$ & $3.464 \mathrm{~km} \mathrm{~s}^{-1}$ \\
$V_{p}$ & $-1 \times 10^{-9} \mathrm{~m} \mathrm{~s}^{-1}$ & $V_{0}$ & $1 \times 10^{-6} \mathrm{~m} \mathrm{~s}^{-1}$ \\
$\sigma_{n}^{0}$ & $50 \mathrm{MPa}$ & $T^{0}$ & $26.5461 \mathrm{MPa}$ \\
$\eta$ & $\rho_{0} \mathrm{c}_{\mathrm{s}} / 2 \mathrm{~Pa} \mathrm{~s} \mathrm{~m}^{-1}$ & $t_{\text {end }}$ & $1500 \mathrm{yr}$ \\
\hline
\end{tabular}

shallowly observed slow slip transients (Wendt et al. 2009; Ikari et al. 2013).

The mechanics of slip on detachment normal faults have been extensively debated. The geologic record shows that shallowly dipping $\left(<30^{\circ}\right)$ crustal-scale detachment faults play a major role in accommodating extension at divergent plate boundaries (Collettini 


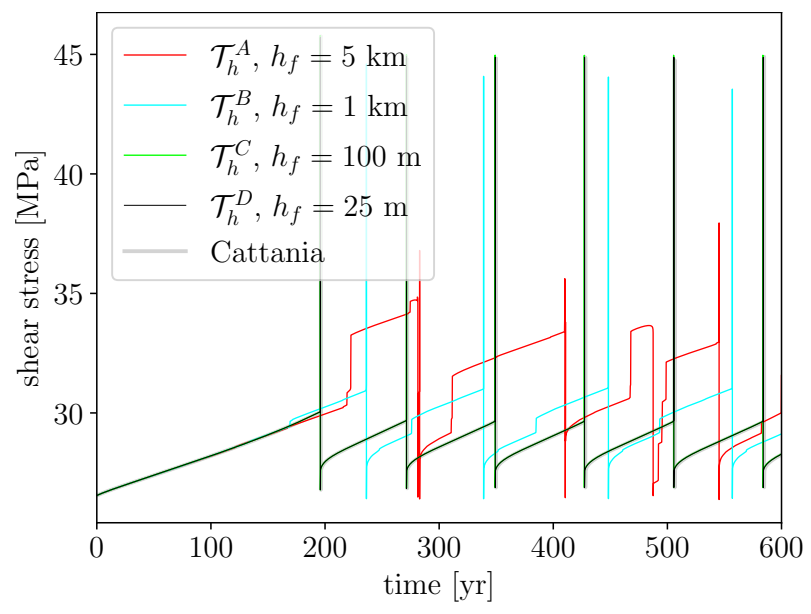

Figure 10. Shear-stress obtained from BP1 on different meshes compared with the reference solution from C. Cattania (Erickson et al. 2020). All experiments used a polynomial degree of $N=2$.

2011, e.g.,). However, such shallowly dipping normal faults appear to challenge the laws of classic static fault mechanical analysis (e.g., Fletcher et al. 2016; Anderlini et al. 2016; Webber et al. 2018; Biemiller et al. 2020, 2021). Furthermore, detachment systems can often be complex with more steeply dipping splay-faults rendering the hanging wall. Such splay faults may slip co-seismically posing seismic and tsunami hazard or relieve shallow interseismic stress on detachments by subsidiary creep. We demonstrate the potential of our method to model seismic and aseismic slip naturally interacting across a main fault with multiple, geometrically complex splay faults. Similar fault system geometries are also ubiquitous in subduction zone megathrust fault systems (Park et al. 2002; Waldhauser et al. 2012), where splay fault slip trading off with slip to the trench is thought to play an important role in tsunamigenesis (Gao et al. 2018; Aslam et al. 2021; Ulrich et al. 2022; van Zelst et al. 2021).

We create a fault system model consisting of a planar, $\delta_{0}=$ $20^{\circ}$ dipping main normal fault and four curved splays faults, as shown in the sketch in Figure 13a. For each splay fault three control points are defined that would model a $50^{\circ}$ and a $40^{\circ}$ dipping

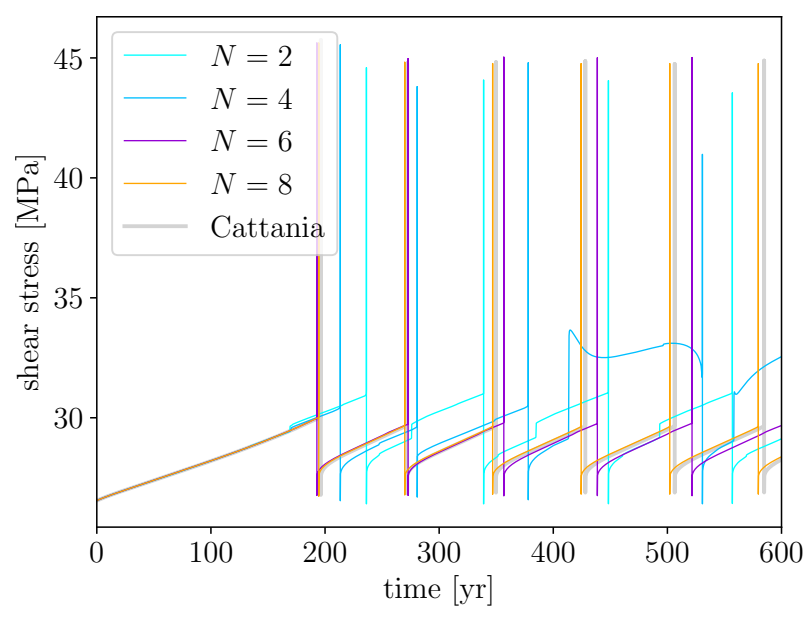

Figure 11. Shear-stress obtained from BP1 on mesh $\mathcal{T}_{h}^{B}\left(h_{f}=1 \mathrm{~km}\right)$ as a function of polynomial degree $N=2,4,6,8$ compared with the reference solution from C. Cattania (Erickson et al. 2020).
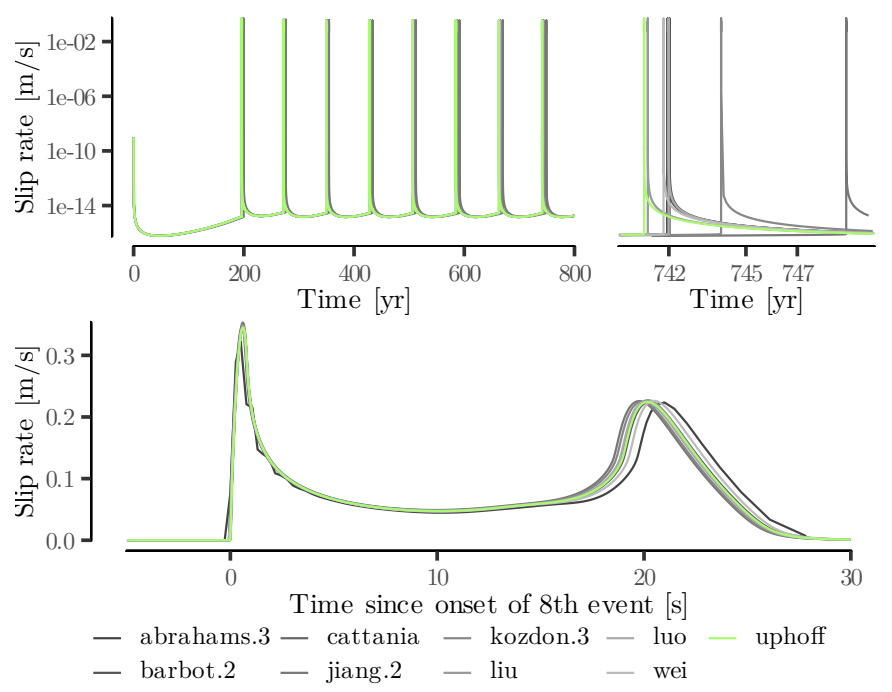

Figure 12. Comparison of the BP1 problem to Erickson et al. (2020, Figures 5 and 6). Top left: Evolution of slip rate over 800 years at $7.5 \mathrm{~km}$ depth. Top right: Detail view of the eighth event. The onset time of the event deviates largely between codes. Bottom: We shift the time-series by their onset time of the eighth event. The onset time is here defined as the first time when the slip-rate is greater or equal than $0.001 \mathrm{~m} \mathrm{~s}^{-1}$.

fault segment when connected by straight lines. Here, we define the splay faults as the spline interpolant of the three control points (using Gmsh's Spline geometry object). The fault system is embedded in the $\left(X_{0}, X_{1}\right) \times\left(Y_{0}, Y_{1}\right)$ box, where $X_{0}=-1000 \mathrm{~km}$, $X_{1}=1000 \mathrm{~km}\left(1+\cos \left(\delta_{0}\right) / \sin \left(\delta_{0}\right)\right), Y_{0}=-1000 \mathrm{~km}$, and $Y_{1}=0 \mathrm{~km}$. The origin of the main normal fault coincides with the origin of the coordinate system and the splays are labelled according to their offsets given by $30 \mathrm{~km}, 50 \mathrm{~km}, 70 \mathrm{~km}$, and $90 \mathrm{~km}$, respectively. With $V_{p}$ negative, cf. Table 6 , the domain is under extension and we model normal faulting. Natural boundary conditions are imposed on the top $\left(y=Y_{0}\right)$ and bottom $\left(y=Y_{1}\right)$ boundary of the model domain. On the left $\left(x=X_{0}\right.$ ) boundary we impose $u_{1}=V_{p} t / 2, u_{2}=0 \mathrm{~m}$ and on the right $\left(x=X_{1}\right)$ boundary we impose $u_{1}=-V_{p} t / 2, u_{2}=0 \mathrm{~m}$.

We adopt a piece-wise linear parametrisation of rate and state friction law parameters $a-b$ as proposed by Rice (e.g., 1993), resembling intact granite (Blanpied et al. 1991). We hold $b$ constant and let $a$ depend on depth by piece-wise linear interpolation of the points $\left(0 \mathrm{~km}, a_{\max }\right),\left(4 \mathrm{~km}, a_{0}\right),\left(15 \mathrm{~km}, a_{0}\right)$, and $\left(18 \mathrm{~km}, a_{\max }\right)$. We hold $a$ constant at $a_{\max }$ below a depth of $18 \mathrm{~km}$. Given $a_{0}$, $a_{\max }$, and $b$ as in Table 6 , we have velocity weakening behaviour in $(8 / 3 \mathrm{~km}, 16 \mathrm{~km})$ and velocity strengthening above and beneath that depth range. All material parameters and other parameters of the rate and state model are constant and given in Table 6 .

We follow a refinement strategy analogue to Section 8.3, and set the on-fault resolution to $h_{f}=0.5 \mathrm{~km}$ and the far boundary resolution to $h=100 \mathrm{~km}$ for a total of 46721 triangles. The polynomial degree is set to $N=8$ and all five faults are also approximated with a degree 8 polynomial. We note that we ran the scenario for on-fault resolutions $h_{f} \in\{4 \mathrm{~km}, 2 \mathrm{~km}, 1 \mathrm{~km}, 0.5 \mathrm{~km}\}$. Going from $h_{f}=2 \mathrm{~km}$ to $0.5 \mathrm{~km}$ there is little difference in the number of events, the timing of events, and the peak slip-rate.

Our exemplary results in Figure 13b and Figure 13c illustrate 1400 years of slip and slip rate evolution on the main low-angle normal fault and all four curved splay faults in response to steady tectonic loading. Note, that we here do not omit a potential "spin-up 


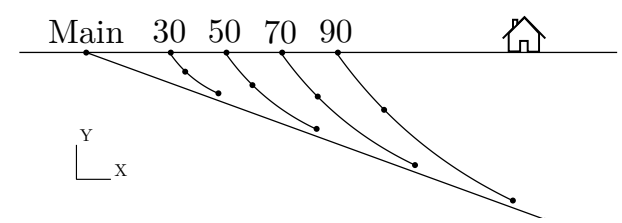

(a) Sketch of the low-angle normal fault and splay fault geometry model. Numbers indicate the offset of each splay fault in kilometre in the $x$-direction w.r.t. the intersection of the main shallowly dipping normal fault with the free surface.

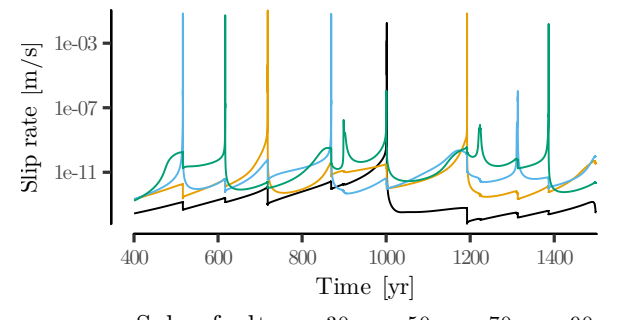

Splay fault $-30-50-70-90$

(b) Time series of slip-rate at $10 \mathrm{~km}$ depth for each splay fault.

Figure 13. Results of the low-angle normal fault SEAS scenario with four curved splay faults, sketched in (a). We observe an irregular series of small and large events at all splay faults as shown in (b). The complete evolution of slip and slip-rate over 1500 years is shown in (c), where a slip contour is drawn whenever the maximum slip-rate changed by $10 \%$ (or more). Red areas indicate a seismic event and light blue areas indicate creeping at plate rate.

phase", and thus, the shown early events may not be fully representative of the longer-term behavior. We observe complex slip evolution and fault interaction throughout the seismic cycle expressed in irregular fast and slow events occurring on the splay faults while the main fault predominantly creeps at plate rate. While we here assume mostly uniform initial conditions (in all parameters besides $a$ ), a more variable setup for example in normal stress may yield both megathrust and splay fault rupture.

The shortest splay, closest to the main fault at $30 \mathrm{~km}$, shows regular low-slip rate events and produces a single fast earthquake. It accumulates roughly half of the long-term slip compared to the two largest splays. For the splays in $50 \mathrm{~km}$ and $70 \mathrm{~km}$ distance to the surface expression of the main fault, we find preliminary indications for slip, slip rate and recurrence rate correlating with splay fault length. Such apparent correlation breaks down for the largest splay, set furthest apart from the main fault at $90 \mathrm{~km}$ distance, which hosts regular aseismic transients and seismic events which magnitude appears to decrease over time. It is offset by roughly the same amount of slip as its shorter neighboring splay fault. In our exemplary model setup, all splays are able to co-seismically slip up to the free surface. Aseismic transients appear at $90 \mathrm{~km}$ and less pronounced at $70 \mathrm{~km}$ distance only after initial earthquakes on those splays. Interestingly, we observe rather complex splay fault coupling (due to static and quasi-dynamic stress transfer). For example, an earthquake rupturing the shortest splay after $\approx 1000$ years, triggers the highest co-seismic slip-rate on the longest, furthest away splay fault. While we refrain from further quantitative analysis here due to the demonstration scope of this example, we note that future work can readily use our setup for geophysical analysis of the seismic cycle in low-angle normal fault as well as megathrust-splay fault networks by adapting the loading conditions.

In the scenario presented here, we leave an arbitrarily small gap between the main fault and the splays following an equivalent strategy established for branching fault geometries in dynamic earthquake rupture code verification exercises (Pelties et al. 2014; Harris et al. 2018). We note that whilst intersecting faults can be handled by our elasticity solver, cf. Section 9.2, when running a SEAS model with intersecting faults $\left(h_{f}=2 \mathrm{~km}\right)$, we observe that shortly after $t=10 \mathrm{yr}$ the normal stress becomes tensile. Inspecting the results, see Figure 14a, reveals that strain becomes very large at the intersection between a splay and the main fault, and that the change in traction changes sign, indicated by red lines which show the fault system geometry warped by the change in shear traction in the direction of the normal of the main fault. We might overcome this issue by future implementation of appropriate jump conditions for tensile stresses (Day et al. 2005) or accounting for off-fault inelastic deformation (Templeton \& Rice 2008; Gabriel et al. 2013; Erickson et al. 2017; Wollherr et al. 2018; Mia et al. 2021).

We repeated the scenario with non-intersecting faults on an affine mesh (degree 1) in which the splay faults are approximated by linear elements. Figure 14b shows artifacts in both the strain and traction (the latter indicated as red lines). These artifacts coincide with the vertices, indicating that they are caused by the abrupt change in angle of the fault normal. The rate and state simulation aborts after $t=100 \mathrm{yr}$ due to unrealistically high stresses likely caused by the ODE solver becoming unstable. In comparison, when using a curvilinear mesh, we observe no artifacts in strain and traction at $t=100 \mathrm{yr}$, cf. Figure 14c.

Lastly, we report the application run-times on a dual socket AMD EPYC 7662 server using 120 processes, the fifth order Dormand-Prince scheme with six stages, and the linear solver combining p-multigrid and algebraic multigrid. For the $h_{f}=4 \mathrm{~km}$ mesh, computing the discrete Green's function took approximately $0.8 \mathrm{~h}$ and the time integration took $1.5 \mathrm{~h}$. In comparison, the run without discrete Green's function took $31 \mathrm{~h}$ showing that the precomputation step clearly pays off for this application. Computation of the discrete Green's function on the finest mesh $\left(h_{f}=0.5 \mathrm{~km}\right)$, 


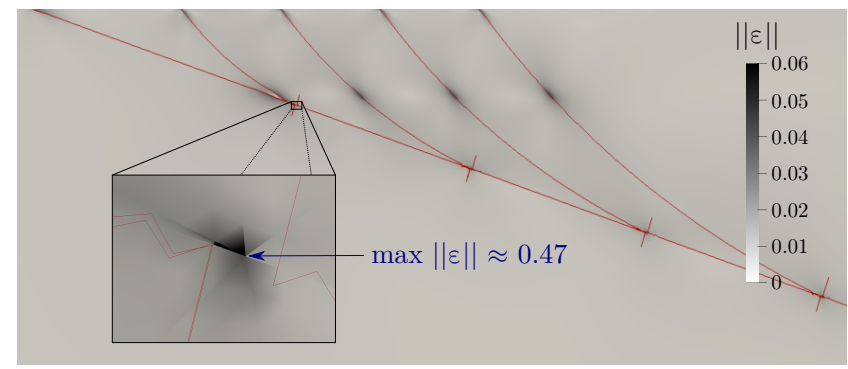

(a) Splays intersecting the main fault cause large strain at the intersection point.

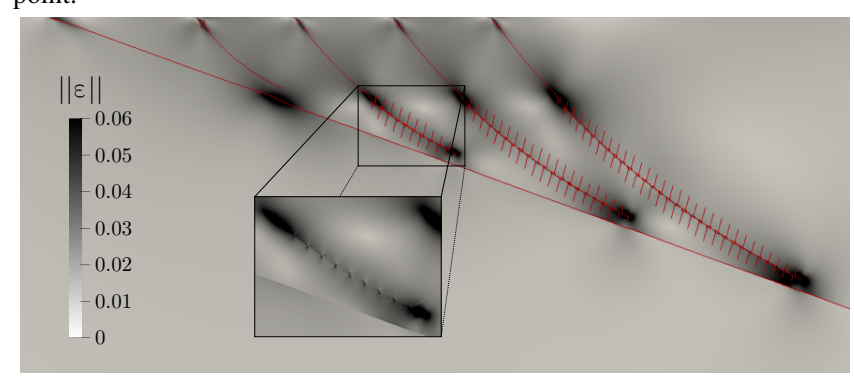

(b) Artifacts in strain and traction are visible on an affine mesh (nonintersecting) due to the linear approximation of the boundary.

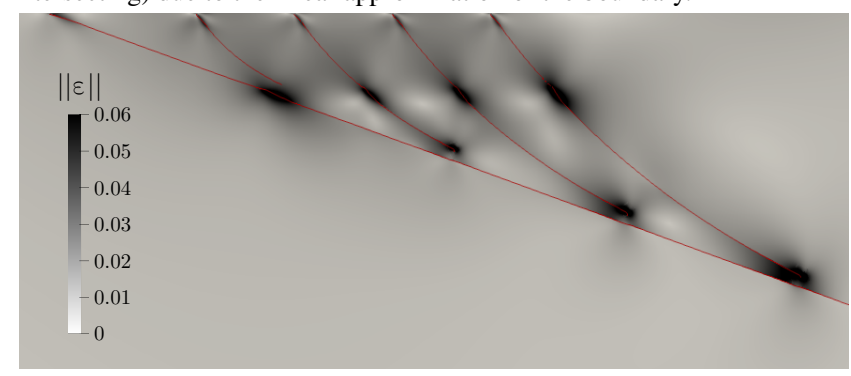

(c) No visible artifacts with non-intersecting faults and curvilinear mesh.

Figure 14. Variations of the scenario with four curved splay faults. Red lines show the fault system warped by the change in shear traction in the direction of the normal of the main fault. The Frobenius norm of the strain tensor is shown in black and white. The on-fault mesh size is $h_{f}=2 \mathrm{~km}$. (a) The elasticity solver can handle intersections of splay and main fault, but the rate and state solver fails due to large strain at the intersection point ( $t=$ $10 \mathrm{yr}$ ). (b) The use of affine meshes causes severe artifacts in traction (red lines, see below) and strain at $(t=100 \mathrm{yr})$. The rate and state solver fails, too. (c) Non-intersecting faults with curvilinear meshes causes no visible artifacts $(t=100 \mathrm{yr})$ and is well handled by the elasticity and rate state solver.

using the same configuration and hardware, took $1.1 \mathrm{~d}$ and time integration took $0.9 \mathrm{~d}$ for a total of 963676 time-steps and 45474 time-step rejections.

\subsection{A 3D multi-fault scenario of instantaneous displacement due to the 2019 Ridgecrest earthquake sequence}

In our next, kinematic elasto-static demonstration example, we consider an idealized instantaneous displacement scenario due to the multi-fault 2019 Ridgecrest, California earthquake sequence. This foreshock-mainshock pair of large events occurred on a segmented antithetic fault network dominated by northwest-trending rightlateral and northeast-trending left-lateral strike-slip (e.g., Ross et al. 2019; Chen et al. 2020; Fialko \& Jin 2021). The Ridgecrest sequence has produced vigorous postseismic crustal motions (e.g.,
Dorsett et al. 2019; Pollitz et al. 2021). Modelling viscous relaxation requires accurate initial conditions, such as provided exemplary in the following.

Our demonstration model considers (simplified) distinct strike-slip displacements accumulated during both events across six geometrically complex and intersecting fault segments, inspired by data-constrained 3D dynamic rupture modeling of both events (Taufiqurrahman et al. 2019). The example geometry of the Ridgecrest fault system has been simplified from integrated geodetic InSAR data, satellite imagery, relocated seismicity, and selected focal mechanisms (Carena \& Suppe 2002; Taufiqurrahman et al. 2019).

The model domain is $130 \mathrm{~km} \times 135 \mathrm{~km}$ in map view and $50 \mathrm{~km}$ deep. The fault network is centrally located within the model domain. All faults are assumed to be purely vertical and extending to a depth of $11 \mathrm{~km}$ (DuRoss et al. 2020). The domain size is chosen such that the faults are $\sim 50 \mathrm{~km}$ from the side walls and $39 \mathrm{~km}$ from the lower boundary. The geometry of each fault is represented by a Bezier spline provided by Gmsh's BSpline geometry object. We impose zero displacement (all components) on the lateral walls, and on the upper and lower boundaries we impose zero stress (in both tangential and normal directions). We drive displacement in the domain by imposing slip along each of the six fault segments. Along each fault, we prescribe constant slip to produce either pure left-lateral or right-lateral (depending on the fault) strike-slip motion. Faults with strike tending NW-SE are assumed to slip right-lateral, while those tending NE-SW are taken to be left-lateral (Barnhart et al. 2020). A schematic of the fault system with the imposed slip boundary conditions is shown in Figure 15. We further assume that the material is homogeneous with a Poisson ratio of $\nu=0.25$ and shear modulus $\mu=36 \mathrm{GPa}$.

The mesh is constructed such that the spatial resolution (element edge length) on the faults is $250 \mathrm{~m}$. We further specified that the spatial resolution on the domain boundaries be $20 \mathrm{~km}$. With these specifications, the mesh generated contained 421,154 tetrahedra. A zoom (with cut-away) of the local mesh refinement near the fault system is shown in Figure 16. In all experiments, we used the multigrid preconditioner described in Section 5.3. For simulations employing $N=1$ (the case where polynomial coarsening cannot be invoked), the multigrid preconditioner reduces to using the algebraic multigrid package GAMG from PETSc.

The instantaneous displacement obtained using a polynomial of degree $N=3$ for the displacement unknown and a non-affine mesh geometry with polynomial degree $N=2$ is shown in Figure 17. Due to the imposed values of slip with $S_{1}>S_{k}, k=$ $2, \ldots, 6$, the displacement is primarily dominated by the slip from segment $S_{1}$ (see Figure 15). We note that despite the simplicity in terms of initial slip conditions of our demonstration model, the resulting displacements are in overall agreement (qualitatively) with more advanced inferences (e.g., Fialko \& Jin 2021).

The discontinuous nature of the displacement approximation is apparent when inspecting the horizontal components $u_{0}, u_{1}$ (Figure 17 (a), (b)). This is expected and consistent with the imposed slip boundary conditions. What is note worthy is that no numerical artefacts appear at the intersecting points of segments $S_{3}-S_{1}$ and $S_{2}-S_{3}$. In contrast, the vertical component of displacement is nearly continuous due to the purely vertical fault orientation, homogeneous material properties and the pure strike-slip loading. Several components of the displacement gradient are shown in Figure 18. In the upper panels (a), a low degree polynomial was used for the displacement $(N=1)$ with an affine mesh. The lower panels (b) employed a displacement polynomial of $N=3$ with a non-affine 


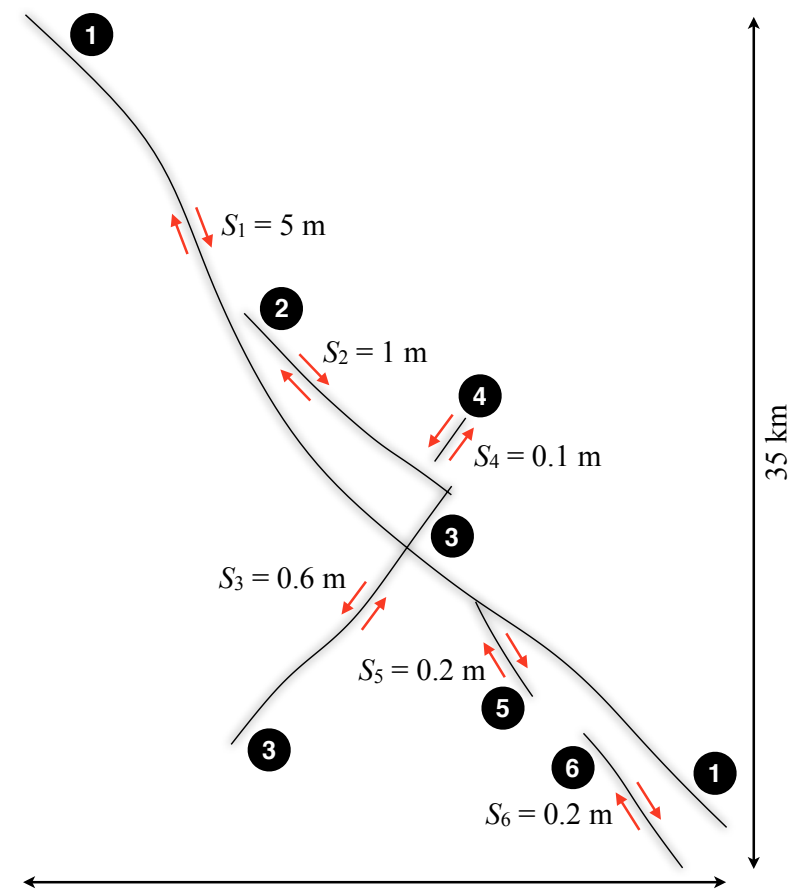

$29 \mathrm{~km}$

Figure 15. Schematic of the Ridgecrest fault system geometry, the sense of slip (red arrows) and values for the imposed strike-slip $\left(S_{k}\right)$ on each fault segment $k=1, \ldots, 6$ in the instantaneous, kinematic elasto-static model. The absolute values used for $S_{k}$ (i.e. ignoring the sense of slip) were $5.0,1.0,0.6,0.1,0.2,0.2 \mathrm{~m}$.

geometry representation using $N=2$. For the $N=1$ scenario, the slip vector imposed is defined such that it is tangential to the affine cell facets which approximate the fault boundary. In the high-order scenario, since the fault is discretised via a curvilinear representation, the slip imposed is tangential to the Bezier spline line used to represent the fault geometry.

Comparing the gradient fields between (a) and (b) we note that while the discontinuities are captured by the low order displacement solution $\left(u_{0, x}, u_{0, y}, u_{0, z}\right)$ significant numerical oscillations close to the fault (thin black line) are apparent. These oscillations vanish when using the higher degree displacement and geometry representation.

It is also striking that the low order discretisation is quite inaccurate in approximating the smooth field $u_{0, z}$. This is partially exaggerated by the fact that we constructed the mesh with coarse resolution off-fault; regardless, the benefits of using high-order approximations is evident: The high-order simulations resolve the singularity in the strain tensor which occurs at the end of each fault. While such singularities are the result of our idealized and somewhat unphysical model (i.e. the slip doesn't taper off towards the end of the fault) our exemplary results demonstrate that the DG method is robust with respect to such loading conditions which might occur (and be physically meaningful): (i) when a fault intersects the free surface or terminates at a material interface with increased rigidity; (ii) when two faults intersect each other; (iii) at the location of a fault branch. Additionally we highlight that no special treatment was required to accommodate the fault intersections (i.e. between segments 1-3 and segments 2-3) - the DG solution, and its gradient, is well behaved at these locations.

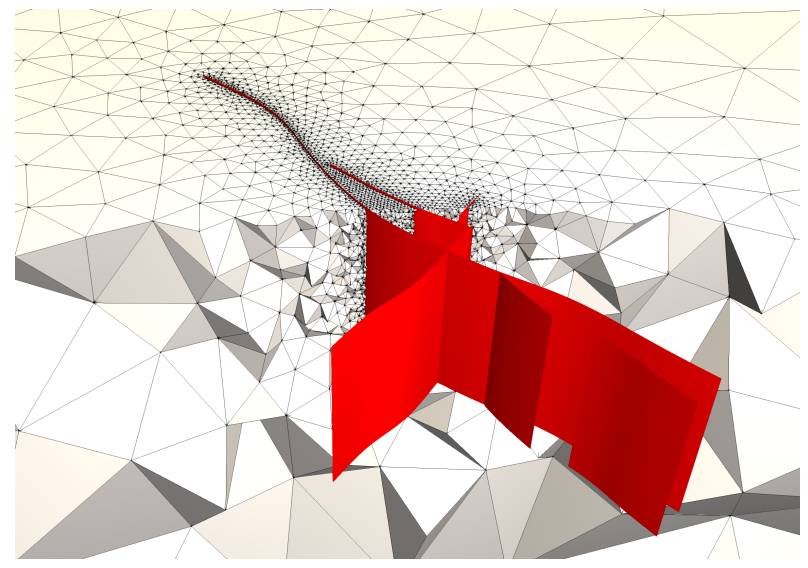

Figure 16. Close up and cut-away of the unstructured tetrahedral mesh used for the Ridgecrest example. The local refinement near the fault results in element edge lengths of $250 \mathrm{~m}$. Away from the fault, the element edge length is coarsened up to a value of $20 \mathrm{~km}$ at the domain boundary. The mesh contained a total of 421,154 tetrahedra.

\section{DISCUSSION}

In the following, we discuss the numerical results with respect to high-order convergence, give a qualitative comparison to the boundary element method, and describe how an extension to a fully-dynamic SEAS modelling method might be obtained.

\subsection{A high-order method for seismic cycling problems}

We measure high-order convergence rates for elastostatic problems with a slip boundary condition, curved boundary, heterogeneous material parameters, and for the time-dependent manufactured SEAS problem, cf. Sections 8.1 and 8.2. For smooth problems the high-order convergence pays off as the error decays much quicker in comparison to low-order rates, cf. the discussion in Section 8.2 and Figure 7.

A requirement for high-order convergence is that the solution is sufficiently smooth, meaning that higher-order (weak) derivatives exist. We see in Figure 3 that the convergence order is limited to 0.5 in the $|.|_{1, h}$ semi-norm, due to a singularity at the origin in the second derivative for the case $\gamma=0.5$. Therefore, the results in Figure 7 model only the case ideal for high-order methods. In actual SEAS problems, the smoothness of the solution is unknown.

For non-smooth solutions, we observe that the error is still reduced by a constant factor when comparing a higher-order method to a lower-order method (cf. Wollherr et al. (2018)'s discussion for dynamic rupture problems), thus the additional degrees of freedom per element of the higher-order method are not in vain. Moreover, in combination with unstructured meshes we can locally refine the mesh. That is, small mesh sizes can be used in non-smooth regions while large mesh sizes are sufficient in smooth regions, see Figure 9.

\subsection{A qualitative comparison of DG versus BEM}

The use of boundary integral equations is widespread in the SEAS community (Lapusta et al. 2000; Lapusta \& Liu 2009; Liu \& Rice 2005; Segall \& Bradley 2012; Bradley 2014; Li \& Liu 2016, 2017; Luo et al. 2017; Barbot 2019) and is also used in earlier work (e.g., Rice 1993). Here, we qualitatively compare the presented DG method to BEM for SEAS applications. 


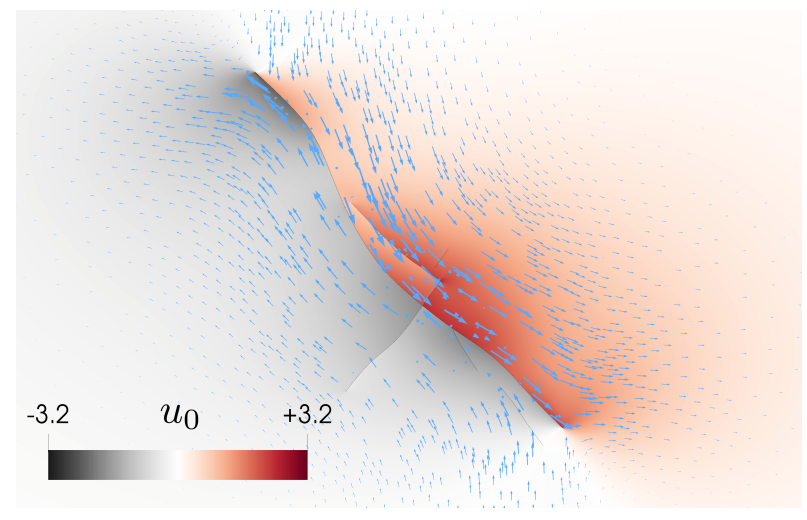

(a) $x$ displacement $\left(u_{0}\right)$

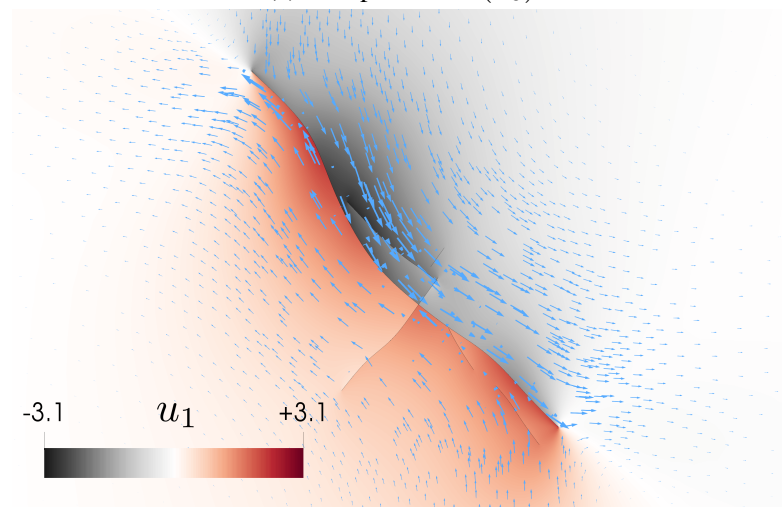

(b) $y$ displacement $\left(u_{1}\right)$

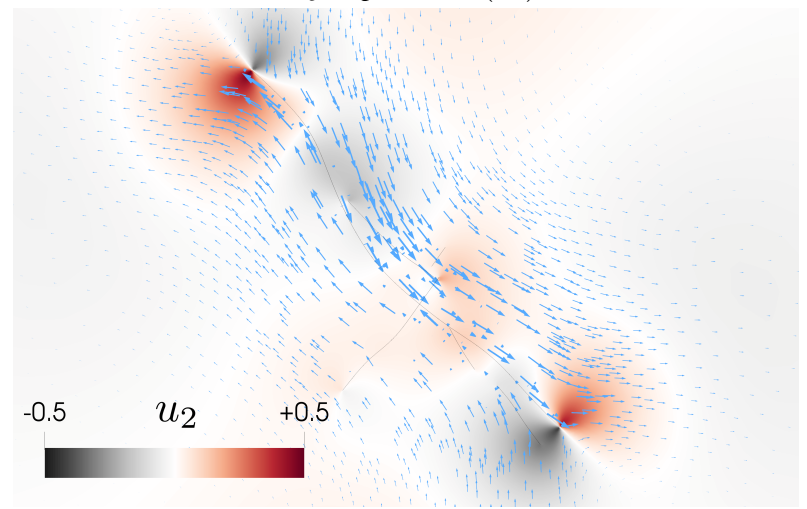

(c) $z$ displacement $\left(u_{2}\right)$

Figure 17. Displacement field in the vicinity of the fault network. (a), (b): Horizontal components ( $x, y$ respectively) of the displacement. (c) Vertical component of the displacement field. All panels are overlaid with vectors denoting the horizontal components of the displacement field $\left(u_{0}, u_{1}\right)$. The faults are indicated by the thin black lines.

We first recall background and definitions of the BEM. For elastostatics in homogeneous and isotropic media, a necessary ingredient is the problem

$\mu \frac{\partial^{2} E_{i j}\left(\boldsymbol{x}^{\prime}, \boldsymbol{x}\right)}{\partial x_{k} \partial x_{k}}+(\lambda+\mu) \frac{\partial^{2} E_{k j}\left(\boldsymbol{x}^{\prime}, \boldsymbol{x}\right)}{\partial x_{k} \partial x_{i}}=-\delta\left(\boldsymbol{x}^{\prime}-\boldsymbol{x}\right) \delta_{i j}$.

The tensor $E$ that solves (87) is called the fundamental solution or Kelvin's solution and is well-known (Chen \& Zhou 2010). The columns of $E$ are interpreted as the displacement at $\boldsymbol{x}^{\prime}$ due to a point force at $\boldsymbol{x}$. From the fundamental solution one obtains the Betti-Somigliana equation (Chen \& Zhou 2010), i.e. for any inter- nal point $\boldsymbol{x}^{\prime} \in \Omega$ we have

$u_{i}\left(\boldsymbol{x}^{\prime}\right)=\int_{\partial \Omega} E_{i j}\left(\boldsymbol{x}^{\prime}, \boldsymbol{x}\right)\left[t_{j}(\boldsymbol{u})\right](\boldsymbol{x})-T_{i j}\left(\boldsymbol{x}^{\prime}, \boldsymbol{x}\right) u_{j}(\boldsymbol{x}) \mathrm{d} s_{\boldsymbol{x}}$,

where $\boldsymbol{t}$ is the traction vector given $\boldsymbol{u}$ and the $j$-th column of $T$ is the traction vector given the $j$-th column of $E$. Equation (88) is the basis for deriving BEMs with slip boundary condition (e.g., Portela et al. 1992), but the details are not important for this discussion.

Inspecting (88) reveals that we need both Dirichlet and Neumann data on the boundary in order to compute $\boldsymbol{u}$. As we typically know either one or the other, (88) can be slightly adapted such that it is valid for $\boldsymbol{x}^{\prime} \in \partial \Omega$ (Portela et al. 1992; Chen \& Zhou 2010). Then we can discretise the boundary and solve the boundary integral equations for the discrete traction given Dirichlet data (or vice versa when Neumann data is given).

Eventually, both the DG method and the BEM require the solution to a linear system $\boldsymbol{A} \underline{\boldsymbol{u}}=\boldsymbol{b}$, although the matrices $\boldsymbol{A}$ are quite different. For DG, $\boldsymbol{A}$ is sparse and, assuming a uniform grid, the number of degrees of freedom scales with $n_{1}^{D}$, where $n_{1}$ is the number of line elements. For BEM, $\boldsymbol{A}$ is dense and the number of degrees of freedom scales with $n_{1}^{D-1}$, because only the boundary needs to be discretised. Recalling that the complexity of exactly solving (e.g by LU factorisation) an $n \times n$ sparse linear system is $\mathcal{O}\left(n^{2}\right)$ whilst the complexity for an $n \times n$ dense linear system is $\mathcal{O}\left(n^{3}\right)$, we can estimate a complexity of $\mathcal{O}\left(n_{1}^{2 D}\right)$ for DG and a complexity of $\mathcal{O}\left(n_{1}^{3(D-1)}\right)$ for BEM. That is, we observe that BEM has a lower complexity in $2 \mathrm{D}$, but actually the complexity is identical in $3 \mathrm{D}$, at least using this simple estimate.

In the previous paragraph we provided pessimistic bounds which would result from straight-forward implementations of the DG method and BEM. Specializations of specific components of each method exist which reduce the algorithmic complexity and potentially alter the cost of each approach. We outline several enhancements below:

- The complexity of solving the sparse DG system can be reduced to $\mathcal{O}(n)$ when iterative solvers employing multigrid are used. However, in practice achieving perfect $\mathcal{O}(n)$ scaling is somewhat problem dependent and hard to achieve in a consistent manner across a wide range of polynomial degrees (e.g., from $N=2-8$ ).

- For BEM, a H-matrix approximation (Bradley 2014) might reduce the storage and solve cost (Hackbusch \& Khoromskij 2000).

- The cost of evaluating the integral operator (e.g. a matrixvector product) required by integral methods can be reduced to $\mathcal{O}(n)$ using the fast-multipole method (FMM) (Rokhlin 1985; Greengard \& Rokhlin 1987; Carrier et al. 1988; Romanet et al. 2018). Additionally, large scale parallel implementations of FMM exist (Cruz et al. 2011; Yokota et al. 2011). We note that while FMM provides fast application of the kernels, it does not define a "solve" (e.g., $\boldsymbol{A} \underline{\boldsymbol{u}}=\boldsymbol{b}$ ). Hence when a FMM-BEM formulation requires the solution of a linear system, a preconditioner will be required to reduce the time complexity.

- Using the approach of the spectral boundary integral method, the cost of evaluating the integral operator can be reduced to $\mathcal{O}(n \log n)$ (per fault segment) as stress interactions are evaluated in the Fourier domain and these computations can exploit a fast Fourier transform (FFT) (Geubelle \& Rice 1995; Perrin et al. 1995; Bouchon \& Streiff 1997; Lapusta \& Liu 2009; Barbot 2021). Note that this specialization does limit the methods applicability to planar faults. Similarly to FMM (above), this fast integral operator evaluation does not replace the need for a solve.

In practice, one needs to consider many more aspects, e.g. which 
(a)
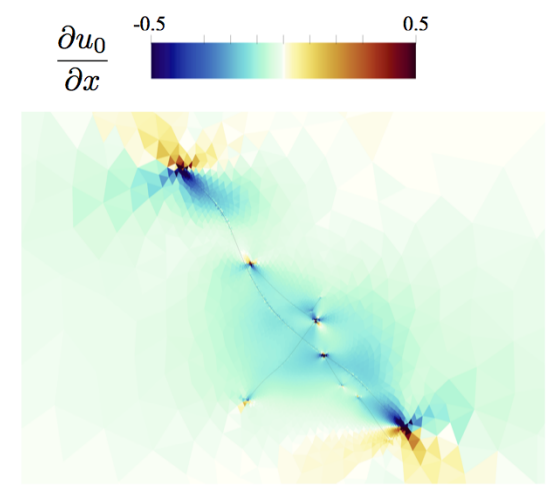

(b)

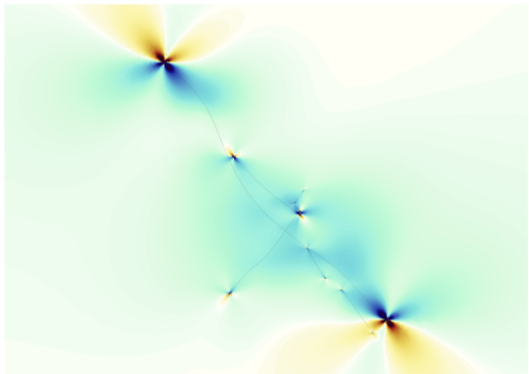

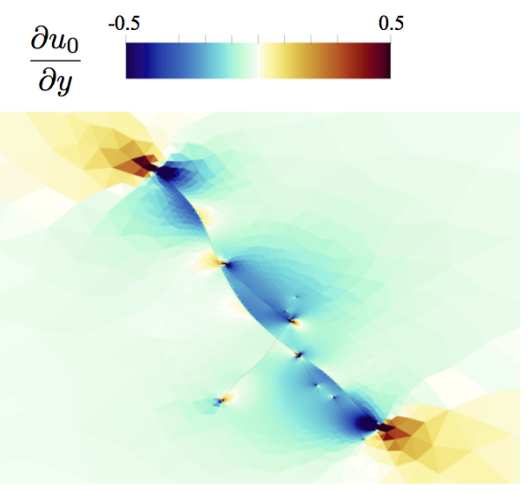
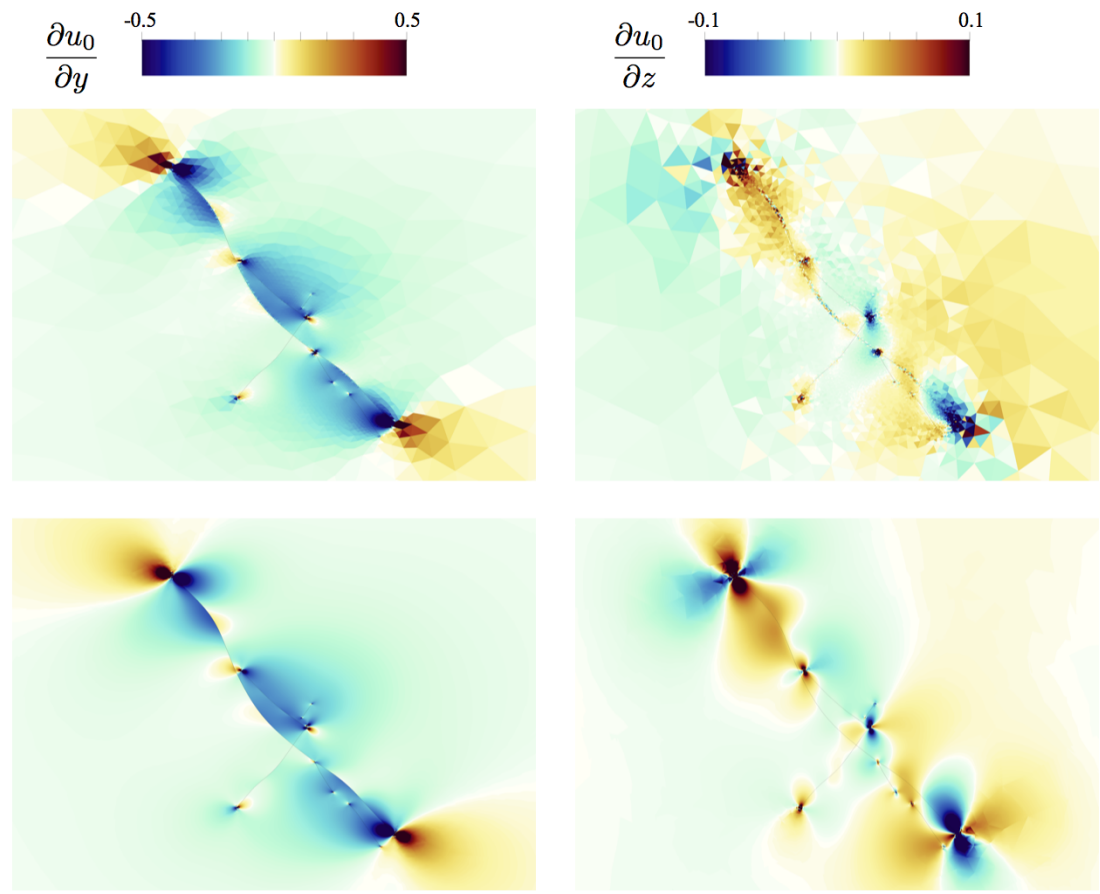

Figure 18. Gradient of the $x$-component of displacement $\left(u_{0}\right)(x, y$-horizontal directions, $z$ - vertical direction) in the vicinity of the fault network using different polynomial approximations. (a) $\boldsymbol{u}_{h}$ approximated with polynomial degree $N=1$ over an affine mesh (fault geometry given by $N=1$ ). (b) $\boldsymbol{u}_{h}$ approximated with polynomial degree $N=3$ on a non-affine mesh with the fault geometry approximated by a polynomial of degree $N=2$. The faults are indicated by the thin black lines.

parts of the method can be parallelized (Amdahl's law, Amdahl (1967)), what are the memory requirements, or whether the method is suitable for current hardware architectures. We conclude that based on above considerations a fair comparison of DG and BEM is rendered inherently application-specific and it is thus not a priori obvious which method will yield the optimal time-to-solution.

There is, however, a special case which makes BEM very costeffective for specific model setups. Assume that Dirichlet data is given and we are missing Neumann data. Further assume that we can find a tensor field $\boldsymbol{G}$ (i.e. a Green's function) such that $\boldsymbol{G}$ is a fundamental solution on $\Omega$ and $G_{i j}=0$ if $\boldsymbol{x} \in \partial \Omega$. Then the first term in (88) vanishes and the Neumann data is not required anymore such that $\boldsymbol{u}$ is computed just by integration. In a discrete setting, the solution is thus obtained by (dense) matrix-vector multiplication only. Hence for a single Green's function, this multiplication has a time complexity of $\mathcal{O}\left(n^{2}\right)$. As mentioned earlier, this can be reduced in general to $\mathcal{O}(n)$ using FMM, or when applicable, to $\mathcal{O}(n \log n)$ via FFT. The use of analytical Green's function is widely adopted in the SEAS community (Lapusta \& Liu (2009, Eq. 5); Liu \& Rice (2005, Eq. 1); Li \& Liu (2016, Eq. 5); Segall \& Bradley (2012, Eq. 1); Barbot (2019, Eq. 8)).

The difficulty in the analytical Green's function approach lies in the dependence of the Green's function on the boundary. That is, whenever the fault geometry changes, multiple faults are introduced (e.g. fault networks or splay faults), or when topography is introduced, then another Green's function is needed. While analytical Green's functions for a single planar fault in the elastic halfspace are known (Okada 1992), as well as those for multiple parallel faults in the elastic half-space (Barbot 2021), finding analytical Green's function for complex fault systems or topography is a formidable task. Thus, in the following we assume that the analytical Green's function approach does not generalise to arbitrarily complex models and one has to resort to BEM, which involves the solution of a large and dense system of equations.

The computational cost of a time-step using the discrete Green's function, cf. Section 7, is comparable to the cost of a timestep using analytical Green's function, because only matrix multiplication is needed in both cases. Clearly, the discrete Green's function is expensive to obtain, as a linear system needs to be solved for every degree of freedom on-fault, but they may be computed for any fault geometry, fault network, and topography supported by our method. We note that computing the discrete Green's function is an embarrassingly parallel problem.

\subsection{Extension to a fully-dynamic method}

In this work we adopt the quasi-dynamic approach and neglect inertia. We see this as the first step towards a future SIPG implementation of the fully-dynamic approach. In particular, the bilinear operator of the SIPG method can be directly employed in a wavepropagation - i.e. fully-dynamic - scheme (Mazzieri et al. 2013). Moreover, the linear solvers we develop for the quasi-dynamic approach can be readily adapted for implicit time-stepping in a fullydynamic model.

The extension of the SIPG method for elastostatics to wave propagation problems is straightforward. The elastodynamic equations are given by

$\rho \frac{\partial^{2} u_{i}}{\partial t^{2}}-\frac{\partial \sigma_{i j}(\boldsymbol{u})}{\partial x_{j}}=f_{i}$.

The SIPG method for the above equation is formulated as following (Grote et al. 2006; Mazzieri et al. 2013): Find $\boldsymbol{u}_{h} \in\left[0, t_{\text {end }}\right] \times$ 
$V_{h} \rightarrow \mathbb{R}$ such that

$\int_{\Omega} \rho \boldsymbol{v} \cdot \frac{\partial^{2} \boldsymbol{u}_{h}}{\partial t^{2}} \mathrm{~d} \boldsymbol{x}+a\left(\boldsymbol{u}_{h}, \boldsymbol{v}\right)=L(\boldsymbol{v})$,

for all $\boldsymbol{v} \in V_{h}, t \in\left[0, t_{\text {end }}\right]$, where the bilinear form $a(.,$.$) and$ the linear form $L($.$) are defined in (23). Written in terms of linear$ algebra, we have

$\boldsymbol{M} \frac{\partial^{2} \underline{u}}{\partial t^{2}}+\boldsymbol{A} \underline{\boldsymbol{u}}=\boldsymbol{b}$

where $\boldsymbol{A}$ and $\boldsymbol{b}$ are constructed identically as in Section 5, and $\boldsymbol{M}$ is the block-diagonal mass matrix weighted with the density $\rho$. Lowstorage schemes for the inverse mass matrix are discussed by Warburton (2013) and Chan et al. (2017) for curvilinear meshes.

Explicit time-stepping is subject to a CFL condition (Grote et al. 2006), rendering explicit time-stepping infeasible for the time spans considered in SEAS models. One might circumvent the timestep restrictions by switching between fully-dynamic solvers in the co-seismic phase and quasi-dynamic solvers in the aseismic phase (Lapusta et al. 2000; Duru \& Dunham 2016; Romanet \& Ozawa 2021). Using the same finite element space for both solvers is convenient as there is no need for interpolation.

Another possibility might be to treat the elastodynamic equation implicitly. For illustration, the classic Newmark scheme leads to the linear system that needs to be solved in every time step (Hughes 2000, Eq. 9.1.12)

$\left(\boldsymbol{M}+\beta \Delta t^{2} \boldsymbol{A}\right) \underline{\boldsymbol{a}}^{n+1}=\boldsymbol{b}^{n+1}-\boldsymbol{A} \underline{\widetilde{\boldsymbol{u}}}^{n+1}$,

where the predictor $\widetilde{\boldsymbol{u}}^{n+1}$ depends only on time-step $n$. Here, linear solvers developed for the elastostatic problem can likely be reused, e.g. the multigrid solver, cf. Section 5.3. Whether such a scheme yields time-steps reasonable for SEAS models remains to be explored.

\section{CONCLUSIONS}

We present a symmetric interior penalty discontinuous Galerkin (SIPG) method to perform quasi-dynamic simulations of sequences of earthquakes and aseismic slip (SEAS). Through an extensive suite of analytic, manufactured, and code-verification examples for elasto-static and seismic cycle problems, we characterise the solution behaviour of the DG discretisation. Our verification suite demonstrates that high-order convergence of the discrete solution can be achieved in space (and time) for both elasto-static and SEAS problems when the polynomial degree of the displacement is increased. We demonstrate that high-order convergence is maintained when either the material properties vary within a cell, or the fault or exterior boundary is curved provided both the coefficients and geometry are discretised with the same polynomial degree as used for the displacement. We exploit the curvilinear geometry representation in two demonstrator models to further elucidate the importance of using curvilinear fault representations, compared with piece-wise linear approximations (affine), when accurate stresses (or displacement gradients) are required on-fault.

We provide an open-source reference implementation, Tandem, which supports both 2D and 3D simulations of the seismic cycle, accounting for geologic heterogeneities and geometric complexity and arbitrary polynomial degree for the displacement, material properties and geometry. Tandem natively supports node-level and distributed memory parallelism. With awareness of the high computational demands of SEAS type problems, our reference implementation exploits modern, efficient libraries for the DG kernels and linear algebra, solver and preconditioner support. Further flexibility and efficiency is provided by optionally allowing users to define the displacement evaluation via the discrete Green's function, which are evaluated and checkpoint once in an embarrassingly parallel pre-computation step using algorithmically optimal and scalable sparse parallel solvers and preconditioners. This strategy appears to exploit advantages of both the boundary integral and volumetric methods and is an interesting avenue to pursue in the future for extreme scale 3D SEAS simulations.

\section{ACKNOWLEDGMENTS}

This work has received funding from the European Research Council (ERC) under the European Union's Horizon 2020 research and innovation programme (TEAR (www.tear-erc.eu), Grant No. 852992, and additional support from ChEESE, Grant No. 823844).

This work has also received support by the National Science Foundation (NSF Grant No. EAR-2121666), the Southern California Earthquake Center (SCEC Grant No. 21112), the German Research Foundation (DFG Grant No. GA 2465/2-1, Grant No. GA 2465/3-1) and the Bavarian Competence Network for Technical and Scientific High Performance Computing (KONWIHR project NewWave).

The authors gratefully acknowledge the Gauss Centre for Supercomputing e.V. (www.gauss-centre.eu) for providing computing time on the GCS Supercomputer SuperMUC-NG at Leibniz Supercomputing Centre (www . Irz. de through project pr63qo). Additional computing resources were provided by the Institute of Geophysics of LMU Munich (Oeser et al. 2006).

We thank James Biemiller, Taufiqurrahman, Nico Schliwa and Duo Li for helpful discussion of demonstration examples.

Author contribution statement: Project conceptualisation: AAG, DAM. Mathematical development: CU, DAM. Software development: CU, DAM. Conceived and designed the experiments: CU, DAM, AAG. Performed the experiments: CU, DAM. Analysed the results: CU, DAM, AAG. Drafted the manuscript: CU, DAM, AAG. Procured funding, project administration and supervision: AAG.

\section{DATA AVAILABILITY}

The Jupyter notebook, setups, and data are openly available in Zenodo at https://doi.org/10.5281/zenodo.5796104. Instructions on how to repeat the presented numerical experiments are also contained in the Zenodo dataset. The code is available in GitHub at https: //github. com/TEAR-ERC/tandem. Instructions on how to build and run the code are available at https://tandem.readthedocs.io. The setups in Section 8 are alternatively available from https://github.com/TEAR-ERC/ tandem/blob/master/examples/. The Jupyter notebook which details the steps to construct the solution in Section 8.1.1 is alternatively available at https://github.com/TEAR-ERC/tandem/ $\mathrm{blob} / \mathrm{main} /$ notebooks/wedge. ipynb.

\section{REFERENCES}

Aagaard, B. T., Knepley, M. G., \& Williams, C. A., 2013. A domain decomposition approach to implementing fault slip in finite-element models of quasi-static and dynamic crustal deformation, Journal of Geophysical Research: Solid Earth, 118(6), 3059-3079. 
Abdelmeguid, M., Ma, X., \& Elbanna, A., 2019. A novel hybrid finite element-spectral boundary integral scheme for modeling earthquake cycles: Application to rate and state faults with low-velocity zones, Journal of Geophysical Research: Solid Earth, 124(12), 12854-12881.

Abhyankar, S., Brown, J., Constantinescu, E. M., Ghosh, D., Smith, B. F., \& Zhang, H., 2018. PETSc/TS: A modern scalable ODE/DAE solver library, Tech. rep., arXiv:1806.01437.

Allison, K. L. \& Dunham, E. M., 2018. Earthquake cycle simulations with rate-and-state friction and power-law viscoelasticity, Tectonophysics, 733, 232-256, Physics of Earthquake Rupture Propagation.

Almquist, M. \& Dunham, E. M., 2021. Elastic wave propagation in anisotropic solids using energy-stable finite differences with weakly enforced boundary and interface conditions, Journal of Computational Physics, 424, 109842.

Amdahl, G. M., 1967. Validity of the single processor approach to achieving large scale computing capabilities, in Proceedings of the April 1820, 1967, Spring Joint Computer Conference, AFIPS '67 (Spring), p. 483-485, Association for Computing Machinery, New York, NY, USA. Amestoy, P. R., Duff, I. S., L'Excellent, J.-Y., \& Koster, J., 2001. A fully asynchronous multifrontal solver using distributed dynamic scheduling, SIAM Journal on Matrix Analysis and Applications, 23(1), 15-41.

Amestoy, P. R., Buttari, A., L'Excellent, J.-Y., \& Mary, T., 2019. Performance and scalability of the block low-rank multifrontal factorization on multicore architectures, ACM Trans. Math. Softw., 45(1).

Anderlini, L., Serpelloni, E., \& Belardinelli, M. E., 2016. Creep and locking of a low-angle normal fault: Insights from the Altotiberina fault in the Northern Apennines (Italy), Geophysical Research Letters, 43(9), 43214329.

Ando, R. \& Kaneko, Y., 2018. Dynamic rupture simulation reproduces spontaneous multifault rupture and arrest during the $2016 \mathrm{Mw} 7.9$ Kaikoura earthquake, Geophysical Research Letters, 45(23), 12,875-12,883.

Antonietti, P. F. \& Houston, P., 2011. A class of domain decomposition preconditioners for hp-discontinuous Galerkin finite element methods, Journal of Scientific Computing, 46(1), 124-149.

Arioli, M., Demmel, J. W., \& Duff, I. S., 1989. Solving sparse linear systems with sparse backward error, SIAM Journal on Matrix Analysis and Applications, 10(2), 165-190.

Arndt, D., Fehn, N., Kanschat, G., Kormann, K., Kronbichler, M., Munch, P., Wall, W. A., \& Witte, J., 2020. ExaDG: High-order discontinuous Galerkin for the exa-scale, in Software for Exascale Computing-SPPEXA 2016-2019, pp. 189-224, Springer, Cham.

Arnold, D. N., Brezzi, F., Cockburn, B., \& Marini, L. D., 2002. Unified analysis of discontinuous Galerkin methods for elliptic problems, SIAM Journal on Numerical Analysis, 39(5), 1749-1779.

Aslam, K. S., Thomas, A. M., \& Melgar, D., 2021. The effect of fore-arc deformation on shallow earthquake rupture behavior in the cascadia subduction zone, Geophysical Research Letters, 48(20), e2021GL093941.

Balay, S., Gropp, W. D., McInnes, L. C., \& Smith, B. F., 1997. Efficient management of parallelism in object oriented numerical software libraries, in Modern Software Tools in Scientific Computing, pp. 163202, Birkhäuser Press.

Balay, S., Abhyankar, S., Adams, M. F., Brown, J., Brune, P., Buschelman, K., Dalcin, L., Dener, A., Eijkhout, V., Gropp, W. D., Karpeyev, D., Kaushik, D., Knepley, M. G., May, D. A., McInnes, L. C., Mills, R. T., Munson, T., Rupp, K., Sanan, P., Smith, B. F., Zampini, S., Zhang, H., \& Zhang, H., 2021a. PETSc users manual, Tech. Rep. ANL-95/11 Revision 3.15, Argonne National Laboratory.

Balay, S., Abhyankar, S., Adams, M. F., Brown, J., Brune, P., Buschelman, K., Dalcin, L., Dener, A., Eijkhout, V., Gropp, W. D., Karpeyev, D., Kaushik, D., Knepley, M. G., May, D. A., McInnes, L. C., Mills, R. T., Munson, T., Rupp, K., Sanan, P., Smith, B. F., Zampini, S., Zhang, H., \& Zhang, H., 2021b. PETSc Web page, https://petsc.org/. Barber, J. R., 2004. Elasticity, Kluwer Academic Publishers, 2nd edn. Barbot, S., 2019. Slow-slip, slow earthquakes, period-two cycles, full and partial ruptures, and deterministic chaos in a single asperity fault, Tectonophysics, 768, 228171.

Barbot, S., 2021. A spectral boundary-integral method for quasi-dynamic ruptures of multiple parallel faults, Bulletin of the Seismological Society of America, 111(3), 1614-1630.

Barbot, S., Lapusta, N., \& Avouac, J.-P., 2012. Under the hood of the earthquake machine: Toward predictive modeling of the seismic cycle, Science, 336(6082), 707-710.

Barnhart, W. D., Gold, R. D., \& Hollingsworth, J., 2020. Localized faultzone dilatancy and surface inelasticity of the 2019 Ridgecrest earthquakes, Nature Geoscience, 13(10), 699-704.

Ben-Menahem, A., 1991. Four thousand years of seismicity along the Dead Sea rift, Journal of Geophysical Research: Solid Earth, 96(B12), 20195-20216.

Biemiller, J., Boulton, C., Wallace, L., Ellis, S., Little, T., Mizera, M., Niemeijer, A., \& Lavier, L., 2020. Mechanical Implications of Creep and Partial Coupling on the World's Fastest Slipping Low-Angle Normal Fault in Southeastern Papua New Guinea, Journal of Geophysical Research: Solid Earth, 125(10), e2020JB020117.

Biemiller, J. B., Gabriel, A.-A., \& Ulrich, T., 2021. The Dynamics of Unlikely Slip: 3D Modeling of Low-angle Normal Fault Rupture at the Mai'iu Fault, Papua New Guinea, Earth and Space Science Open Archive, p. 36.

Blanpied, M. L., Lockner, D. A., \& Byerlee, J. D., 1991. Fault stability inferred from granite sliding experiments at hydrothermal conditions, Geophysical Research Letters, 18(4), 609-612.

Bouchon, M. \& Streiff, D., 1997. Propagation of a shear crack on a nonplanar fault: A method of calculation, Bulletin of the Seismological Society of America, 87(1), 61-66.

Bradley, A. M., 2014. Software for Efficient Static Dislocation-Traction Calculations in Fault Simulators, Seismological Research Letters, 85(6), 1358-1365.

Brenner, S. C., 2004. Korn's inequalities for piecewise $H^{1}$ vector fields, Mathematics of Computation, 73(247), 1067-1087.

Brezzi, F. \& Fortin, M., 1991. Mixed and Hybrid Finite Element Methods, Springer, New York, NY.

Buiter, S. J., Schreurs, G., Albertz, M., Gerya, T. V., Kaus, B., Landry, W., Le Pourhiet, L., Mishin, Y., Egholm, D. L., Cooke, M., et al., 2016. Benchmarking numerical models of brittle thrust wedges, Journal of Structural Geology, 92, 140-177.

Carena, S. \& Suppe, J., 2002. Three-dimensional imaging of active structures using earthquake aftershocks: the Northridge thrust, California, Journal of Structural Geology, 24(4), 887-904.

Carrier, J., Greengard, L., \& Rokhlin, V., 1988. A fast adaptive multipole algorithm for particle simulations, SIAM journal on scientific and statistical computing, 9(4), 669-686.

Castillo, P., Cockburn, B., Perugia, I., \& Schötzau, D., 2000. An a priori error analysis of the local discontinuous Galerkin method for elliptic problems, SIAM Journal on Numerical Analysis, 38(5), 1676-1706.

Chan, J., Hewett, R. J., \& Warburton, T., 2017. Weight-adjusted discontinuous Galerkin methods: Curvilinear meshes, SIAM Journal on Scientific Computing, 39(6), A2395-A2421.

Chen, G. \& Zhou, J., 2010. Boundary Element Methods with Applications to Nonlinear Problems, Atlantis Press, 2nd edn.

Chen, K., Avouac, J.-P., Aati, S., Milliner, C., Zheng, F., \& Shi, C., 2020. Cascading and pulse-like ruptures during the 2019 Ridgecrest earthquakes in the Eastern California Shear Zone, Nature Communications, 11(1), 22.

Cockburn, B. \& Shu, C. W., 1989. TVB Runge-Kutta local projection discontinuous Galerkin finite element method for conservation laws II: general framework, Mathematical Computation, 52, 411-435.

Collettini, C., 2011. The mechanical paradox of low-angle normal faults: Current understanding and open questions, Tectonophysics, 510(3), 253268.

Cruz, F. A., Knepley, M. G., \& Barba, L. A., 2011. PetFMM-a dynamically load-balancing parallel fast multipole library, International journal for numerical methods in engineering, 85(4), 403-428.

Day, S. M., Dalguer, L. A., Lapusta, N., \& Liu, Y., 2005. Comparison of finite difference and boundary integral solutions to three-dimensional spontaneous rupture, Journal of Geophysical Research: Solid Earth, 110(B12).

Dieterich, J. H., 1979. Modeling of rock friction: 1. Experimental re- 
sults and constitutive equations, Journal of Geophysical Research: Solid Earth, 84(B5), 2161-2168.

Dieterich, J. H. \& Kilgore, B. D., 1994. Direct observation of frictional contacts: New insights for state-dependent properties, Pure and Applied Geophysics, 143(1), 283-302.

Dieterich, J. H., Richards-Dinger, K. B., \& Kroll, K. A., 2015. Modeling injection-induced seismicity with the physics-based earthquake simulator RSQSim, Seismological Research Letters, 86(4), 1102-1109.

Dorsett, J., Johnson, K. M., Puel, S., \& Becker, T. W., 2019. Postseismic deformation and stress evolution following the 2019 M7.1 and M6.4 Ridgecrest earthquakes, in AGU Fall Meeting Abstracts, vol. 2019, pp. S31G-0500.

Dumbser, M. \& Käser, M., 2006. An arbitrary high-order discontinuous Galerkin method for elastic waves on unstructured meshes - II. The three-dimensional isotropic case, Geophysical Journal International, 167(1), 319-336.

DuRoss, C. B., Gold, R. D., Dawson, T. E., Scharer, K. M., Kendrick, K. J., Akciz, S. O., Angster, S. J., Bachhuber, J., Bacon, S., Bennett, S. E. K., Blair, L., Brooks, B. A., Bullard, T., Burgess, W. P., Chupik, C., DeFrisco, M., Delano, J., Dolan, J. F., Frost, E., Graehl, N., Haddon, E. K., Hatem, A. E., Hernandez, J. L., Hitchcock, C., Hudnut, K., Thompson Jobe, J., Koehler, R., Kozaci, O., Ladinsky, T., Madugo, C., McPhillips, D. S., Milliner, C., Morelan, A., Olson, B., Patton, J., Philibosian, B., Pickering, A. J., Pierce, I., Ponti, D. J., Seitz, G., Spangler, E., Swanson, B., Thomas, K., Treiman, J., Valencia, F., Williams, A., \& Zinke, R., 2020. Surface Displacement Distributions for the July 2019 Ridgecrest, California, Earthquake Ruptures, Bulletin of the Seismological Society of America, 110, 1400-1418.

Duru, K. \& Dunham, E. M., 2016. Dynamic earthquake rupture simulations on nonplanar faults embedded in 3D geometrically complex, heterogeneous elastic solids, Journal of Computational Physics, 305, 185207.

Duru, K., Rannabauer, L., Gabriel, A.-A., Ling, O. K. A., Igel, H., \& Bader, M., 2022. A stable discontinuous Galerkin method for linear elastodynamics in 3D geometrically complex elastic solids using physics based numerical fluxes, Computer Methods in Applied Mechanics and Engineering, 389, 114386.

Epshteyn, Y. \& Rivière, B., 2007. Estimation of penalty parameters for symmetric interior penalty Galerkin methods, Journal of Computational and Applied Mathematics, 206(2), 843-872.

Erickson, B. A. \& Dunham, E. M., 2014. An efficient numerical method for earthquake cycles in heterogeneous media: Alternating subbasin and surface-rupturing events on faults crossing a sedimentary basin, Journal of Geophysical Research: Solid Earth, 119(4), 3290-3316.

Erickson, B. A., Dunham, E. M., \& Khosravifar, A., 2017. A finite difference method for off-fault plasticity throughout the earthquake cycle, Journal of the Mechanics and Physics of Solids, 109, 50-77.

Erickson, B. A., Jiang, J., Barall, M., Lapusta, N., Dunham, E. M., Harris, R., Abrahams, L. S., Allison, K. L., Ampuero, J., Barbot, S., Cattania, C., Elbanna, A., Fialko, Y., Idini, B., Kozdon, J. E., Lambert, V., Liu, Y., Luo, Y., Ma, X., Best McKay, M., Segall, P., Shi, P., van den Ende, M., $\&$ Wei, M., 2020. The community code verification exercise for simulating sequences of earthquakes and aseismic slip (SEAS), Seismological Research Letters, 91(2A), 874-890.

Fialko, Y. \& Jin, Z., 2021. Simple shear origin of the cross-faults ruptured in the 2019 Ridgecrest earthquake sequence, Nature Geoscience, pp. 16.

Fletcher, J. M., Oskin, M. E., \& Teran, O. J., 2016. The role of a keystone fault in triggering the complex El Mayor-Cucapah earthquake rupture, Nature Geoscience, 9(4), 303-307.

Forsythe, G. E., Malcolm, M. A., \& Moler, C. B., 1977. Computer Methods for Mathematical Computations, Prentice Hall.

Gabriel, A.-A., Ampuero, J.-P., Dalguer, L. A., \& Mai, P. M., 2013. Source Properties of Dynamic Rupture Pulses with Off-Fault Plasticity, Journal of Geophysical Research: Solid Earth, 118(8), 4117-4126.

Galvez, P., Somerville, P., Petukhin, A., Ampuero, J.-P., \& Peter, D., 2020. Earthquake cycle modelling of multi-segmented faults: Dynamic rupture and ground motion simulation of the $1992 \mathrm{Mw} 7.3$ Landers earthquake,
Pure and Applied Geophysics, 177(5), 2163-2179.

Gao, D., Wang, K., Insua, T. L., Sypus, M., Riedel, M., \& Sun, T., 2018. Defining megathrust tsunami source scenarios for northernmost Cascadia, Natural Hazards, 94(1), 445-469.

Geubelle, P. H. \& Rice, J. R., 1995. A spectral method for threedimensional elastodynamic fracture problems, Journal of the Mechanics and Physics of Solids, 43(11), 1791-1824.

Geuzaine, C. \& Remacle, J.-F., 2009. Gmsh: A 3-d finite element mesh generator with built-in pre- and post-processing facilities, International Journal for Numerical Methods in Engineering, 79(11), 1309-1331.

Greengard, L. \& Rokhlin, V., 1987. A fast algorithm for particle simulations, Journal of computational physics, 73(2), 325-348.

Grote, M. J., Schneebeli, A., \& Schötzau, D., 2006. Discontinuous Galerkin finite element method for the wave equation, SIAM Journal on Numerical Analysis, 44(6), 2408-2431.

Hackbusch, W. \& Khoromskij, B., 2000. A sparse H-matrix arithmetic: general complexity estimates, Journal of Computational and Applied Mathematics, 125(1), 479-501.

Harris, R. A., Barall, M., Aagaard, B., Ma, S., Roten, D., Olsen, K., Duan, B., Liu, D., Luo, B., Bai, K., et al., 2018. A suite of exercises for verifying dynamic earthquake rupture codes, Seismological Research Letters, 89(3), 1146-1162.

Harris, R. A., Barall, M., Lockner, D. A., Moore, D. E., Ponce, D. A., Graymer, R. W., Funning, G., Morrow, C. A., Kyriakopoulos, C., \& Eberhart-Phillips, D., 2021. A geology and geodesy based model of dynamic earthquake rupture on the Rodgers Creek-Hayward-Calaveras fault system, California, Journal of Geophysical Research: Solid Earth, 126(3), e2020JB020577, e2020JB020577 2020JB020577.

Heinecke, A., Breuer, A., Rettenberger, S., Bader, M., Gabriel, A.-A., Pelties, C., Bode, A., Barth, W., Liao, X.-K., Vaidyanathan, K., et al., 2014. Petascale high order dynamic rupture earthquake simulations on heterogeneous supercomputers, in SC'14: Proceedings of the International Conference for High Performance Computing, Networking, Storage and Analysis, pp. 3-14, IEEE.

Hesthaven, J. S. \& Warburton, T., 2008. Nodal Discontinuous Galerkin Methods, Springer, New York, USA.

Hill, W. H. R. T. R., 1973. Triangular mesh methods for the neutron transport equation, Technical Report LA-UR-73-479, Los Alamos National Laboratory, Los Alamos, New Mexico, USA.

Hughes, T. J. R., 2000. The Finite Element Method: Linear Static and Dynamic Finite Element Analysis, Dover Publications, New York.

Ikari, M. J., Marone, C., Saffer, D. M., \& Kopf, A. J., 2013. Slip weakening as a mechanism for slow earthquakes, Nature Geoscience, 6(6), 468-472.

Jaśkowiec, J. \& Sukumar, N., 2020. High-order cubature rules for tetrahedra, International Journal for Numerical Methods in Engineering, 121(11), 2418-2436.

Jiang, J., Erickson, B. A., Lambert, V. R., Ampuero, J.-P., Ando, R., Barbot, S. D., Cattania, C., Zilio, L. D., Duan, B., Dunham, E. M., Gabriel, A.-A., Lapusta, N., Li, D., Li, M., Liu, D., Liu, Y., Ozawa, S., Pranger, C., \& van Dinther, Y., 2021. Community-driven code comparisons for three-dimensional dynamic modeling of sequences of earthquakes and aseismic slip (SEAS), Earth and Space Science Open Archive, doi: 10.1002/essoar.10508582.1.

Johnen, A., Remacle, J.-F., \& Geuzaine, C., 2013. Geometrical validity of curvilinear finite elements, Journal of Computational Physics, 233, 359-372.

Kaneko, Y., Ampuero, J.-P., \& Lapusta, N., 2011. Spectral-element simulations of long-term fault slip: Effect of low-rigidity layers on earthquake-cycle dynamics, Journal of Geophysical Research: Solid Earth, 116(B10).

Karypis, G. \& Kumar, V., 1998. A fast and high quality multilevel scheme for partitioning irregular graphs, SIAM Journal on Scientific Computing, 20(1), 359-392.

Kato, N., 2002. Seismic cycle on a strike-slip fault with rate-and statedependent strength in an elastic layer overlying a viscoelastic half-space, Earth, planets and space, 54(11), 1077-1083.

Kozdon, J., 2019. QDESDG, https://github.com/jkozdon/QDESDG. 
Krenz, L., Uphoff, C., Ulrich, T., Gabriel, A.-A., Abrahams, L. S., Dunham, E. M., \& Bader, M., 2021. 3D Acoustic-Elastic Coupling with Gravity: The Dynamics of the 2018 Palu, Sulawesi Earthquake and Tsunami, in Proceedings of the International Conference for High Performance Computing, Networking, Storage and Analysis, SC '21, Association for Computing Machinery, New York, NY, USA.

Kronbichler, M. \& Kormann, K., 2019. Fast matrix-free evaluation of discontinuous galerkin finite element operators, ACM Transactions on Mathematical Software (TOMS), 45(3), 1-40.

Kronbichler, M., Kormann, K., Pasichnyk, I., \& Allalen, M., 2017. Fast matrix-free discontinuous Galerkin kernels on modern computer architectures, in International Supercomputing Conference, pp. 237-255, Springer.

Lapusta, N. \& Liu, Y., 2009. Three-dimensional boundary integral modeling of spontaneous earthquake sequences and aseismic slip, Journal of Geophysical Research: Solid Earth, 114(B9).

Lapusta, N., Rice, J. R., Ben-Zion, Y., \& Zheng, G., 2000. Elastodynamic analysis for slow tectonic loading with spontaneous rupture episodes on faults with rate- and state-dependent friction, Journal of Geophysical Research: Solid Earth, 105(B10), 23765-23789.

Lapusta, N., Dunham, E., Avouac, J.-P., Denolle, M., van Dinther, Y., Faulkner, D., Fialko, Y., Kitajima, H., Lambert, V., Larochelle, S., Aagaard, B., Barbot, S., Becker, T. W., Beeler, N. M., Ben-Zion, Y., Beroza, G. C., Bürgmann, R., Brodsky, E. E., Cattania, C., Duan, B., Elbanna, A. E., Ellsworth, W. L., Erickson, B. A., Gabriel, A.A., Gurnis, M., Harris, R. A., Jiang, J., Hirth, G., Kaneko, Y., Kirkpatrick, J. D., Lay, T., Ma, S., Marone, C., McLaskey, G. C., Meier, M.-A., Niemeijer, A. R., Noda, H., Oglesby, D. D., Olsen, K. B., Rosakis, A. J., Ross, Z. E., Rowe, C. D., Segall, P., Tal, Y., Townend, J., Vidale, J. E., Zhan, Z., \& Zhu, W., 2019. Modeling earthquake source processes: from tectonics to dynamic rupture, Report to the national science foundation, http://www. seismolab. caltech. edu/pdf/MESP_White_Paper_Main_Text_8_March_2019.pdf.

Li, D. \& Liu, Y., 2016. Spatiotemporal evolution of slow slip events in a nonplanar fault model for northern Cascadia subduction zone, Journal of Geophysical Research: Solid Earth, 121(9), 6828-6845.

Li, D. \& Liu, Y., 2017. Modeling slow-slip segmentation in Cascadia subduction zone constrained by tremor locations and gravity anomalies, Journal of Geophysical Research: Solid Earth, 122(4), 3138-3157.

Liu, Y. \& Rice, J. R., 2005. Aseismic slip transients emerge spontaneously in three-dimensional rate and state modeling of subduction earthquake sequences, Journal of Geophysical Research: Solid Earth, 110(B8).

Luo, B., Duan, B., \& Liu, D., 2020. 3D finite-element modeling of dynamic rupture and aseismic slip over earthquake cycles on geometrically complex faults, Bulletin of the Seismological Society of America, 110(6), 2619-2637.

Luo, Y., Ampuero, J. P., Galvez, P., van den Ende, M., \& Idini, B., 2017. QDYN: A Quasi-DYNamic earthquake simulator (v1.1).

Mazzieri, I., Stupazzini, M., Guidotti, R., \& Smerzini, C., 2013. SPEED: SPectral Elements in Elastodynamics with Discontinuous Galerkin: a non-conforming approach for 3D multi-scale problems, International Journal for Numerical Methods in Engineering, 95(12), 991-1010.

Mckay, M. B., Erickson, B. A., \& Kozdon, J. E., 2019. A computational method for earthquake cycles within anisotropic media, Geophysical Journal International, 219(2), 816-833.

Meurer, A., Smith, C. P., Paprocki, M., Čertík, O., Kirpichev, S. B., Rocklin, M., Kumar, A., Ivanov, S., Moore, J. K., Singh, S., Rathnayake, T., Vig, S., Granger, B. E., Muller, R. P., Bonazzi, F., Gupta, H., Vats, S., Johansson, F., Pedregosa, F., Curry, M. J., Terrel, A. R., Roučka, v., Saboo, A., Fernando, I., Kulal, S., Cimrman, R., \& Scopatz, A., 2017. SymPy: Symbolic computing in Python, PeerJ Computer Science, 3, e103.

Mia, M. S., Abdelmeguid, M., \& Elbanna, A., 2021. Self-limiting earthquake dynamics and spatio-temporal clustering of seismicity enabled by off-fault plasticity, EarthArXiv, https://doi.org/10.31223/ X50P8B.

Milner, K. R., Shaw, B. E., Goulet, C. A., Richards-Dinger, K. B., Callaghan, S., Jordan, T. H., Dieterich, J. H., \& Field, E. H., 2021. Toward physics-based nonergodic PSHA: A prototype fully deterministic seismic hazard model for Southern California, Bulletin of the Seismological Society of America, 111(2), 898-915.

Oeser, J., Bunge, H.-P., \& Mohr, M., 2006. Cluster design in the earth sciences: Tethys, in International conference on high performance computing and communications, pp. 31-40, Springer.

Okada, Y., 1992. Internal deformation due to shear and tensile faults in a half-space, Bulletin of the Seismological Society of America, 82(2), 1018-1040.

Park, J.-O., Tsuru, T., Kodaira, S., Cummins, P. R., \& Kaneda, Y., 2002. Splay fault branching along the Nankai subduction zone, Science, 297(5584), 1157-1160.

Pelties, C., Gabriel, A.-A., \& Ampuero, J.-P., 2014. Verification of an ADER-DG method for complex dynamic rupture problems, Geoscientific Model Development, 7(3), 847-866.

Perez-Silva, A., Li, D., Gabriel, A.-A., \& Kaneko, Y., 2021. 3D modeling of long-term slow slip events along the flat-slab segment in the Guerrero seismic gap, Mexico, Geophysical Research Letters, 48(13), e2021GL092968.

Perrin, G., Rice, J. R., \& Zheng, G., 1995. Self-healing slip pulse on a frictional surface, Journal of the Mechanics and Physics of Solids, 43(9), 1461-1495.

Petersen, M. D., Moschetti, M. P., Powers, P. M., Mueller, C. S., Haller, K. M., Frankel, A. D., Zeng, Y., Rezaeian, S., Harmsen, S. C., Boyd, O. S., Field, E. H., Chen, R., Rukstales, K. S., Luco, N., Wheeler, R. L., Williams, R. A., \& Olsen, A. H., 2014. Documentation for the 2014 update of the United States national seismic hazard maps, Tech. rep., U.S. Geological Survey Open-File Report 2014-1091, http://pubs . er.usgs.gov/publication/ofr20141091.

Plesch, A., Shaw, J. H., Benson, C., Bryant, W. A., Carena, S., Cooke, M., Dolan, J., Fuis, G., Gath, E., Grant, L., et al., 2007. Community fault model (CFM) for Southern California, Bulletin of the Seismological Society of America, 97(6), 1793-1802.

Pollitz, F. F., Wicks, C. W., Svarc, J. L., Phillips, E., Brooks, B. A., Murray, M. H., \& Turner, R. C., 2021. Postseismic relaxation following the 2019 Ridgecrest, California, earthquake sequence, Bulletin of the Seismological Society of America.

Portela, A., Aliabadi, M. H., \& Rooke, D. P., 1992. The dual boundary element method: Effective implementation for crack problems, International Journal for Numerical Methods in Engineering, 33(6), 12691287.

Pranger, C., 2021. GARNET, https://bitbucket.org/cpranger/garnet.

Reinarz, A., Charrier, D. E., Bader, M., Bovard, L., Dumbser, M., K. Duru, F. F., Gabriel, A.-A., Gallard, J.-M., Köppel, S., Krenz, L., Rannabauer, L., Rezzolla, L., Samfass, P., Tavelli, M., \& Weinzierl, T., 2020. ExaHyPE: An engine for parallel dynamically adaptive simulations of wave problems, Computational Physics Communications, p. 107251.

Rice, J. R., 1993. Spatio-temporal complexity of slip on a fault, Journal of Geophysical Research: Solid Earth, 98(B6), 9885-9907.

Rice, J. R. \& Ben-Zion, Y., 1996. Slip complexity in earthquake fault models, Proc. Natl. Acad. Sci. USA, 93, 3811-3818.

Rice, J. R. \& Tse, S. T., 1986. Dynamic motion of a single degree of freedom system following a rate and state dependent friction law, Journal of Geophysical Research: Solid Earth, 91(B1), 521-530.

Rivière, B., 2008. Discontinuous Galerkin Methods for Solving Elliptic and Parabolic Equations, Society for Industrial and Applied Mathematics.

Rockwell, T. K., Dawson, T. E., Young Ben-Horin, J., \& Seitz, G., 2015. A 21-event, 4,000-year history of surface ruptures in the Anza seismic gap, San Jacinto fault, and implications for long-term earthquake production on a major plate boundary fault, Pure and Applied Geophysics, 172(5), 1143-1165.

Rognes, M., Kirby, R., \& Logg, A., 2009. Efficient assembly of $H$ (div) and $H$ (curl) conforming finite elements, SIAM J. Sci. Comp., 31(6), 4130-4151.

Rokhlin, V., 1985. Rapid solution of integral equations of classical potential theory, Journal of computational physics, 60(2), 187-207.

Romanet, P. \& Ozawa, S., 2021. Fully dynamic earthquake cycle simulations on a nonplanar fault using the spectral boundary integral element 
method (sBIEM), Bulletin of the Seismological Society of America.

Romanet, P., Bhat, H. S., Jolivet, R., \& Madariaga, R., 2018. Fast and slow slip events emerge due to fault geometrical complexity, Geophysical Research Letters, 45(10), 4809-4819.

Ross, Z. E., Idini, B., Jia, Z., Stephenson, O. L., Zhong, M., Wang, X., Zhan, Z., Simons, M., Fielding, E. J., Yun, S.-H., Hauksson, E., Moore, A. W., Liu, Z., \& Jung, J., 2019. Hierarchical interlocked orthogonal faulting in the 2019 Ridgecrest earthquake sequence, Science, 366(6463), 346-351.

Ruina, A., 1983. Slip instability and state variable friction laws, Journal of Geophysical Research: Solid Earth, 88(B12), 10359-10370.

Schoeder, S., Wall, W. A., \& Kronbichler, M., 2019. ExWave: A high performance discontinuous Galerkin solver for the acoustic wave equation, SoftwareX, 9, 49-54.

Segall, P. \& Bradley, A. M., 2012. Slow-slip evolves into megathrust earthquakes in 2D numerical simulations, Geophysical Research Letters, 39(18).

Segall, P., Rubin, A. M., Bradley, A. M., \& Rice, J. R., 2010. Dilatant strengthening as a mechanism for slow slip events, Journal of Geophysical Research: Solid Earth, 115(B12).

Shahbazi, K., 2005. An explicit expression for the penalty parameter of the interior penalty method, Journal of Computational Physics, 205(2), 401-407.

Tago, J., Cruz-Atienza, V. M., Virieux, J., Etienne, V., \& Sánchez-Sesma, F. J., 2012. A 3D hp-adaptive discontinuous Galerkin method for modeling earthquake dynamics, Journal of Geophysical Research: Solid Earth, 117(B9).

Taufiqurrahman, T., Gabriel, A.-A., Bo, L., Li, D., Wirp, A. S., Ulrich, T., Palgunadi, K. H., Verdecchia, A., Carena, S., \& Mildon, Z. K., 2019. High-resolution integrated dynamic rupture modeling of the 2019 M6.4 Searles Valley and M7.1 Ridgecrest earthquakes, in AGU Fall Meeting 2019, AGU.

Templeton, E. L. \& Rice, J. R., 2008. Off-fault plasticity and earthquake rupture dynamics: 1 . dry materials or neglect of fluid pressure changes, Journal of Geophysical Research: Solid Earth, 113(B9).

Thakur, P., Huang, Y., \& Kaneko, Y., 2020. Effects of low-velocity fault damage zones on long-term earthquake behaviors on mature strike-slip faults, Journal of Geophysical Research: Solid Earth, 125(8).

Thomas, M. Y., Lapusta, N., Noda, H., \& Avouac, J.-P., 2014. Quasidynamic versus fully dynamic simulations of earthquakes and aseismic slip with and without enhanced coseismic weakening, Journal of Geophysical Research: Solid Earth, 119(3), 1986-2004.

Tullis, T. E., Richards-Dinger, K., Barall, M., Dieterich, J. H., Field, E. H., Heien, E. M., Kellogg, L. H., Pollitz, F. F., Rundle, J. B., Sachs, M. K., et al., 2012. Generic earthquake simulator, Seismological Research Letters, 83(6), 959-963.

Ulrich, T., Gabriel, A.-A., Ampuero, J.-P., \& Xu, W., 2019. Dynamic viability of the $2016 \mathrm{Mw} 7.8$ Kaikōura earthquake cascade on weak crustal faults, Nature Communications, 10(1), 1213.

Ulrich, T., Gabriel, A.-A., \& Madden, E. H., 2022. Stress, rigidity and sediment strength control megathrust earthquake and tsunami dynamics, Nature Geoscience, (1), in press.

Uphoff, C., 2020. Flexible model extension and optimisation for earthquake simulations at extreme scales, Dissertation, Technische Universität München, Munich.

Uphoff, C. \& Bader, M., 2020. Yet another tensor toolbox for discontinuous Galerkin methods and other applications, ACM Trans. Math. Softw., 46(4).

Uphoff, C., Rettenberger, S., Bader, M., Madden, E. H., Ulrich, T., Wollherr, S., \& Gabriel, A.-A., 2017. Extreme scale multi-physics simulations of the tsunamigenic 2004 Sumatra megathrust earthquake, in Proceedings of the International Conference for High Performance Computing, Networking, Storage and Analysis, SC '17, Association for Computing Machinery, New York, NY, USA.

van Zelst, I., Rannabauer, L., Gabriel, A.-A., \& van Dinther, Y., 2021. Earthquake rupture on multiple splay faults and its effect on tsunamis, EarthArXiv.

Waldhauser, F., Schaff, D. P., Diehl, T., \& Engdahl, E. R., 2012. Splay faults imaged by fluid-driven aftershocks of the 2004 Mw 9.2 SumatraAndaman earthquake, Geology, 40(3), 243-246.

Warburton, T., 2006. An explicit construction of interpolation nodes on the simplex, J. Eng. Math., 56, 247-262.

Warburton, T., 2013. A low-storage curvilinear discontinuous Galerkin method for wave problems, SIAM Journal on Scientific Computing, 35(4), A1987-A2012.

Warburton, T. \& Hesthaven, J., 2003. On the constants in hp-finite element trace inverse inequalities, Computer Methods in Applied Mechanics and Engineering, 192(25), 2765-2773.

Webber, S., Norton, K., Little, T., Wallace, L., \& Ellis, S., 2018. How fast can low-angle normal faults slip? Insights from cosmogenic exposure dating of the active Mai'iu fault, Papua New Guinea, Geology, 46(3), 227-230.

Wendt, J., Oglesby, D. D., \& Geist, E. L., 2009. Tsunamis and splay fault dynamics, Geophysical Research Letters, 36(15).

Wilcox, L. C., Stadler, G., Burstedde, C., \& Ghattas, O., 2010. A highorder discontinuous Galerkin method for wave propagation through coupled elastic-acoustic media, Journal of Computational Physics, 229, 9373-9396.

Williams, M. L., 2021. Stress singularities resulting from various boundary conditions in angular corners of plates in extension, Journal of Applied Mechanics, 19(4), 526-528.

Witherden, F. \& Vincent, P., 2015. On the identification of symmetric quadrature rules for finite element methods, Computers \& Mathematics with Applications, 69(10), 1232-1241.

Wollherr, S., Gabriel, A.-A., \& Uphoff, C., 2018. Off-fault plasticity in three-dimensional dynamic rupture simulations using a modal Discontinuous Galerkin method on unstructured meshes: Implementation, verification and application, Geophysical Journal International, 214(3), 1556-1584.

Yokota, R., Bardhan, J. P., Knepley, M. G., Barba, L. A., \& Hamada, T., 2011. Biomolecular electrostatics using a fast multipole BEM on up to 512 GPUs and a billion unknowns, Computer Physics Communications, 182(6), 1272-1283.

\section{APPENDIX A: PROOF OF COERCIVITY AND BOUNDEDNESS}

In the following we prove Theorem 4.1. Several definitions are required before we proceed. For matrices $\boldsymbol{A}, \boldsymbol{B}$ let $\boldsymbol{A}: \boldsymbol{B}=$ $A_{i j} B_{i j}$. Given a vector-valued function $\boldsymbol{m}$ and a matrix-valued function $M$, on a facet $e$ we will denote inner products via $\|\boldsymbol{m}\|_{e}^{2}=\int_{e} \boldsymbol{m} \cdot \boldsymbol{m} \mathrm{d} s$ and $\|\boldsymbol{M}\|_{e}^{2}=\int_{e} \boldsymbol{M}: \boldsymbol{M} \mathrm{d} s$. Inner products over a volume $E$ will be denoted as $\|\boldsymbol{m}\|_{E}^{2}=\int_{E} \boldsymbol{m} \cdot \boldsymbol{m} \mathrm{d} \boldsymbol{x}$ and $\|\boldsymbol{M}\|_{E}^{2}=\int_{E} \boldsymbol{M}: \boldsymbol{M} \mathrm{d} \boldsymbol{x}$.

For improved readability of this section, we abbreviate the tensor contraction $c_{i j k l} \partial u_{k} / \partial x_{l}$ with $\boldsymbol{c} \boldsymbol{\nabla} \boldsymbol{u}$. Using $\boldsymbol{\sigma}=\boldsymbol{c} \boldsymbol{\nabla} \boldsymbol{u}$ the bilinear form is

$$
\begin{array}{r}
a(\boldsymbol{u}, \boldsymbol{u})=\sum_{E \in \mathcal{T}_{h}} \int_{E} \boldsymbol{\sigma}: \boldsymbol{\nabla} \boldsymbol{u} \mathrm{d} \boldsymbol{x}+\sum_{e \in \Gamma^{i} \cup \Gamma^{D}} \int_{e} \delta_{e} \llbracket \boldsymbol{u} \rrbracket \cdot \llbracket \boldsymbol{u} \rrbracket \mathrm{d} s \\
-\underset{e \in \Gamma^{i} \cup \Gamma^{D}}{ } \int_{e}\{\boldsymbol{\sigma} \boldsymbol{n} \rrbracket \cdot \llbracket \boldsymbol{u} \rrbracket \mathrm{d} s .
\end{array}
$$

Note that the first two terms are equivalent to $\|\boldsymbol{u}\|_{*}^{2}$, hence it is sufficient to find a lower bound for the last term.

We bound the last term in (A.1) using the Cauchy-Schwarz inequality:

$\int_{e}\{\{\boldsymbol{\sigma} \boldsymbol{n}\} \cdot \llbracket \boldsymbol{u} \rrbracket \mathrm{d} s \leq \|\{\boldsymbol{\sigma} \boldsymbol{n}\}\}\left\|_{e}\right\| \llbracket \boldsymbol{u} \rrbracket \|_{e}$.

Using the definition of the average, the triangle inequality, and re- 
calling that $|\boldsymbol{n}|=1$ we have

$\|\{\boldsymbol{\sigma} \boldsymbol{n}\}\|_{e} \leq \frac{1}{2}\left\|\left.\boldsymbol{c} \boldsymbol{\nabla} \boldsymbol{u}\right|_{E_{1}^{e}}\right\|_{e}+\frac{1}{2}\left\|\left.\boldsymbol{c} \boldsymbol{\nabla} \boldsymbol{u}\right|_{E_{1}^{e}}\right\|_{e}$.

We denote the square root of stiffness tensor $c$ with $c^{1 / 2}$. The square root has the property $c_{i j r s}^{1 / 2} c_{r s k l}^{1 / 2}=c_{i j r s}$. For an isotropic stiffness tensor the square root can be shown to be given by $c_{i j k l}^{1 / 2}=$ $a \delta_{i j} \delta_{k l}+b\left(\delta_{i k} \delta j l+\delta_{i l} \delta_{j k}\right)$, where $\lambda=D a^{2}+4 a b$ and $\mu=2 b^{2}$.

Using the square root $\boldsymbol{c}^{1 / 2}, \boldsymbol{c} \boldsymbol{\nabla} \boldsymbol{u}=\boldsymbol{c} \varepsilon(\boldsymbol{u})$, and property (27) we have on element $E$

$\boldsymbol{c} \nabla \boldsymbol{u}: \boldsymbol{c} \boldsymbol{\nabla} \boldsymbol{u} \leq\left(c_{1}^{E}\right)^{2} \varepsilon(\boldsymbol{u}): \boldsymbol{\varepsilon}(\boldsymbol{u})$.

Conversely, we have

$c_{0}^{E} \varepsilon(\boldsymbol{u}): \varepsilon(\boldsymbol{u}) \leq \nabla \boldsymbol{u}: \boldsymbol{c} \nabla \boldsymbol{u}=\boldsymbol{c}^{1 / 2} \nabla \boldsymbol{u}: \boldsymbol{c}^{1 / 2} \nabla \boldsymbol{u}$.

We proceed with bounding $\|\left\{\{\boldsymbol{\sigma} \boldsymbol{n}\} \|_{e}\right.$ using (A.4). As $\boldsymbol{X}_{E}$ is assumed to be affine we have $\varepsilon(\boldsymbol{u}) \in\left[P_{N-1}(E)\right]^{D \times D}$ and (24) can be applied:

$$
\begin{aligned}
\|\{\boldsymbol{\sigma} \boldsymbol{n}\} & \left.\left.\left\|_{e} \leq \frac{c_{1}^{E_{1}^{e}}}{2}\right\| \boldsymbol{\varepsilon}(\boldsymbol{u})\right|_{E_{1}^{e}}\left\|_{e}+\frac{c_{1}^{E_{2}^{e}}}{2}\right\| \varepsilon(\boldsymbol{u})\right|_{E_{2}^{e}} \|_{e} \\
& \leq \frac{c_{1}^{E_{1}^{e}} \sqrt{\alpha_{e, 1}}}{2}\|\varepsilon(\boldsymbol{u})\|_{E_{e}^{1}}+\frac{c_{1}^{E_{2}^{e}} \sqrt{\alpha_{e, 2}}}{2}\|\varepsilon(\boldsymbol{u})\|_{E_{e}^{2}},
\end{aligned}
$$

where $\alpha_{e, i}:=(N(N-1+D) / D)\left(|e| /\left|E_{e}^{i}\right|\right)$. With (A.5) we have

$\|\{\boldsymbol{\sigma} \boldsymbol{n}\}\|_{e} \leq \frac{\sqrt{\beta_{e, 1}}}{2}\left\|\boldsymbol{c}^{1 / 2} \nabla \boldsymbol{u}\right\|_{E_{e}^{1}}+\frac{\sqrt{\beta_{e, 2}}}{2}\left\|\boldsymbol{c}^{1 / 2} \nabla \boldsymbol{u}\right\|_{E_{e}^{2}}$,

where $\beta_{e, i}:=\alpha_{e, i}\left(c_{1}^{E_{i}^{e}}\right)^{2} / c_{0}^{E_{i}^{e}}$. Similarly, we get for boundary facets

$\int_{e}\left\{\{\boldsymbol{\sigma} \boldsymbol{n}\} \cdot \llbracket \boldsymbol{u} \rrbracket \mathrm{d} s \leq \sqrt{\beta_{e, 1}}\left\|\boldsymbol{c}^{1 / 2} \nabla \boldsymbol{u}\right\|_{E_{e}^{1}}\|\llbracket \boldsymbol{u} \rrbracket\|_{e}\right.$.

Summing over all edges and applying the Cauchy-Schwarz inequality we obtain

$$
\begin{aligned}
& \sum_{e \in \Gamma^{i} \cup \Gamma^{D}} \int_{e}\left\{\boldsymbol{\sigma} \boldsymbol{n} \rrbracket \cdot \llbracket \boldsymbol{u} \rrbracket \mathrm{d} s \leq\left((D+1) \sum_{E}\left\|\boldsymbol{c}^{1 / 2} \boldsymbol{\nabla} \boldsymbol{u}\right\|_{E}^{2}\right)^{1 / 2}\right. \\
& \times\left(\sum_{e \in \Gamma^{i}} \frac{\beta_{e, 1}}{4}\|\llbracket \boldsymbol{u} \rrbracket\|_{e}^{2}+\sum_{e \in \Gamma^{i}} \frac{\beta_{e, 2}}{4}\|\llbracket \boldsymbol{u} \rrbracket\|_{e}^{2}+\sum_{e \in \Gamma^{D}} \beta_{e, 1}\|\llbracket \boldsymbol{u} \rrbracket\|_{e}^{2}\right)^{1 / 2}
\end{aligned}
$$

The inequality $a b \leq \zeta a^{2} / 2+b^{2} /(2 \zeta)$ with $\zeta>0$ (which follows from Young's inequality) is used to obtain the final bound

$$
\begin{aligned}
& \sum_{e \in \Gamma^{i} \cup \Gamma^{D}} \int_{e}\left\{\{\boldsymbol{\sigma} \boldsymbol{n}\} \cdot \llbracket \boldsymbol{u} \rrbracket \mathrm{d} S \leq \frac{\zeta}{2} \sum_{E}\left\|\boldsymbol{c}^{1 / 2} \nabla \boldsymbol{u}\right\|_{E}^{2}\right. \\
& +\frac{D+1}{2 \zeta}\left(\sum_{e \in \Gamma^{i}} \frac{\beta_{e, 1}}{4}\|\llbracket \boldsymbol{u} \rrbracket\|_{e}^{2}+\sum_{e \in \Gamma^{i}} \frac{\beta_{e, 2}}{4}\|\llbracket \boldsymbol{u} \rrbracket\|_{e}^{2}+\sum_{e \in \Gamma^{D}} \beta_{e, 1}\|\llbracket \boldsymbol{u} \rrbracket\|_{e}^{2}\right) .
\end{aligned}
$$

Inserting (A.10) into (A.1) yields

$$
\begin{gathered}
a(\boldsymbol{u}, \boldsymbol{u}) \geq(1-\zeta) \sum_{E}\left\|\boldsymbol{c}^{1 / 2} \boldsymbol{\nabla} \boldsymbol{u}\right\|_{E}^{2} \\
+\sum_{e \in \Gamma^{i}}\left(\delta_{e}-\frac{(D+1) \beta_{e, 1}}{4 \zeta}-\frac{(D+1) \beta_{e, 2}}{4 \zeta}\right)\|\llbracket \boldsymbol{u} \rrbracket\|_{e}^{2} \\
+\sum_{e \in \Gamma_{D}}\left(\delta_{e}-\frac{(D+1) \beta_{e, 1}}{\zeta}\right)\|\llbracket \boldsymbol{u} \rrbracket\|_{e}^{2}
\end{gathered}
$$

such that Theorem 4.1 follows. Corollary 4.1 follows from

$-\int_{e}\{\{\boldsymbol{\sigma} \boldsymbol{n}\}] \cdot \llbracket \boldsymbol{u} \rrbracket \mathrm{d} s \leq\|\{\boldsymbol{\sigma} \boldsymbol{n}\}\|_{e}\|\llbracket \boldsymbol{u} \rrbracket\|_{e}$. 